\title{
GRB hosts through cosmic time
}

\section{VLT/X-Shooter emission-line spectroscopy of $96 \gamma$-ray-burst-selected galaxies at $0.1<z<3.6^{\star, \star \star, \star \star \star \star}$}

\author{
T. Krühler ${ }^{1,2}$, D. Malesani ${ }^{2}$, J. P. U. Fynbo ${ }^{2}$, O. E. Hartoog ${ }^{3}$, J. Hjorth ${ }^{2}$, P. Jakobsson ${ }^{4}$, D. A. Perley ${ }^{5, \star \star \star \star}$, A. Rossi ${ }^{6,7}$, \\ P. Schady ${ }^{8}$, S. Schulze ${ }^{9,10}$, N. R. Tanvir ${ }^{11}$, S. D. Vergani ${ }^{12,13}$, K. Wiersema ${ }^{11}$, P. M. J. Afonso ${ }^{14}$, J. Bolmer ${ }^{8}$, Z. Cano ${ }^{4}$, \\ S. Covino ${ }^{13}$, V. D’Elia ${ }^{15,16}$, A. de Ugarte Postigo ${ }^{17,2}$, R. Filgas ${ }^{18}$, M. Friis ${ }^{4}$, J. F. Graham ${ }^{8}$, J. Greiner ${ }^{8}$, P. Goldoni ${ }^{19}$, \\ A. Gomboc ${ }^{20}$, F. Hammer ${ }^{12}$, J. Japelj ${ }^{20}$, D. A. Kann ${ }^{7,8}$, L. Kaper ${ }^{3}$, S. Klose ${ }^{7}$, A. J. Levan ${ }^{21}$, G. Leloudas ${ }^{22,2}$, \\ B. Milvang-Jensen ${ }^{2}$, A. Nicuesa Guelbenzu ${ }^{7}$, E. Palazzi ${ }^{6}$, E. Pian $^{23,24,25}$, S. Piranomonte ${ }^{14}$, R. Sánchez-Ramírez ${ }^{26,27,17}$, \\ S. Savaglio ${ }^{28,23}$, J. Selsing ${ }^{2}$, G. Tagliaferri ${ }^{13}$, P. M. Vreeswijk ${ }^{21, \dagger}$, D. J. Watson ${ }^{2}$, and D. Xu ${ }^{2}$ \\ (Affiliations can be found after the references)
}

Received 20 December 2014 / Accepted 15 June 2015

\begin{abstract}
We present data and initial results from VLT/X-Shooter emission-line spectroscopy of 96 galaxies selected by long $\gamma$-ray bursts (GRBs) at $0.1<$ $z<3.6$, the largest sample of GRB host spectra available to date. Most of our GRBs were detected by Swift and $76 \%$ are at $0.5<z<2.5$ with a median $z_{\mathrm{med}} \sim 1.6$. Based on Balmer and/or forbidden lines of oxygen, nitrogen, and neon, we measure systemic redshifts, star formation rates (SFR), visual attenuations $\left(A_{V}\right)$, oxygen abundances $(12+\log (\mathrm{O} / \mathrm{H}))$, and emission-line widths $(\sigma)$. We study GRB hosts up to $z \sim 3.5$ and find a strong change in their typical physical properties with redshift. The median SFR of our GRB hosts increases from $S F R_{\mathrm{med}} \sim 0.6 M_{\odot} \mathrm{yr}^{-1}$ at $z \sim 0.6$ up to $S F R_{\text {med }} \sim 15 M_{\odot} \mathrm{yr}^{-1}$ at $z \sim 2$. A higher ratio of [O III]/[O II] at higher redshifts leads to an increasing distance of GRB-selected galaxies to the locus of local galaxies in the Baldwin-Phillips-Terlevich diagram. There is weak evidence for a redshift evolution in $A_{V}$ and $\sigma$, with the highest values seen at $z \sim 1.5\left(A_{V}\right)$ or $z \sim 2(\sigma)$. Oxygen abundances of the galaxies are distributed between $12+\log (\mathrm{O} / \mathrm{H})=7.9$ and $12+\log (\mathrm{O} / \mathrm{H})=9.0$ with a median $12+\log (\mathrm{O} / \mathrm{H})_{\text {med }} \sim 8.5$. The fraction of GRB-selected galaxies with super-solar metallicities is $\sim 20 \%$ at $z<1$ in the adopted metallicity scale. This is significantly less than the fraction of total star formation in similar galaxies, illustrating that GRBs are scarce in high metallicity environments. At $z \sim 3$, sensitivity limits us to probing only the most luminous GRB hosts for which we derive metallicities of $Z \lesssim 0.5 Z_{\odot}$. Together with a high incidence of $Z \sim 0.5 Z_{\odot}$ galaxies at $z \sim 1.5$, this indicates that a metallicity dependence at low redshift will not be dominant at $z \sim 3$. Significant correlations exist between the hosts' physical properties. Oxygen abundance, for example, relates to $A_{V}\left(12+\log (\mathrm{O} / \mathrm{H}) \propto 0.17 \cdot A_{V}\right)$, line width $\left(12+\log (\mathrm{O} / \mathrm{H}) \propto \sigma^{0.6}\right)$, and $\mathrm{SFR}\left(12+\log (\mathrm{O} / \mathrm{H}) \propto S F R^{0.2}\right)$. In the last two cases, the normalization of the relations shift to lower metallicities at $z>2$ by $\sim 0.4$ dex. These properties of GRB hosts and their evolution with redshift can be understood in a cosmological context of star-forming galaxies and a picture in which the hosts' properties at low redshift are influenced by the tendency of GRBs to avoid the most metal-rich environments.
\end{abstract}

Key words. gamma-ray burst: general - galaxies: high-redshift - galaxies: star formation - galaxies: evolution

\section{Introduction}

The extreme luminosities of long $\gamma$-ray bursts (GRBs) and their afterglows (e.g., Gehrels et al. 2009; Kumar \& Zhang 2015, for recent reviews) over the full electromagnetic spectrum make them powerful messengers from the early Universe, a potential

* Based on observations at ESO, Program IDs: 084.A-0260, 084.A-0303, 085.A-0009, 086.B-0954, 086.A-0533, 086.A-0874, 087.A-0055， 087.A-0451， 087.B-0737，088.A-0051， 088.A-0644, 089.A-0067, 089.A-0120, 089.D-0256, 089.A-0868, 090.A-0088, 090.A-0760， 090.A-0825，091.A-0342，091.A-0703，091.A-0877, 091.C-0934, 092.A-0076, 092.A-0124，092.A-0231，093.A-0069, 094.A-0593.

$\star \star$ Tables 1-4 and appendices are available in electronic form at http://www . aanda.org

$\star \star \star$ The reduced spectra are only available at the CDS via anonymous ftp to cdsarc.u-strasbg. fr (130.79.128.5) or via

http://cdsarc.u-strasbg.fr/viz-bin/qcat?J/A+A/581/A125

$\star \star \star \star$ Hubble Fellow.

Benoziyo Fellow. that was identified very early on (e.g., Wijers et al. 1998; Lamb \& Reichart 2000). Coupled with the association of long GRBs with core-collapse supernovae of type Ic (e.g., Galama et al. 1998; Hjorth et al. 2003; Pian et al. 2006), and thus the formation of some massive stars, GRBs provide means of studying starforming galaxies at the highest redshifts (e.g., Chary et al. 2007; Chen et al. 2009; Tanvir et al. 2012), faintest luminosities (e.g., Vreeswijk et al. 2001; Trenti et al. 2012), as well as the global star formation rate (SFR) density up to the epoch of re-ionization (e.g., Kistler et al. 2009; Robertson \& Ellis 2012; Elliott et al. 2012). In particular the information that is obtained through afterglow spectroscopy provides insights of unprecedented detail into the chemical composition of high-redshift galaxies (e.g., Fynbo et al. 2006; Prochaska et al. 2009; Thöne et al. 2013; Cucchiara et al. 2015).

Long $\gamma$-ray bursts, however, are rare objects (e.g., Podsiadlowski et al. 2004; Guetta \& Della Valle 2007). The rate at which GRBs are accurately localized through satellites is around two per week. Number statistics of GRBs are thus orders of magnitude lower than those of other prominent probes of 
the early Universe, notably Lyman-break galaxies (LBGs, e.g., Steidel et al. 1996), and damped-Lyman- $\alpha$ absorbers (DLAs, e.g., Wolfe et al. 2005) along the sightline to quasi-stellar objects (QSOs). Quite similarly, the GRB explosion represents a very rare endpoint of stellar evolution, and could thus be subject to environmental factors enhancing or quenching the GRB rate with respect to star formation. For instance, metallicity often has been discussed as being likely to influence GRBs (e.g., Stanek et al. 2006; Modjaz et al. 2008). Variations in the initial-mass function (IMF) or the fraction of massive stars in tight binaries could also affect the GRB rate (Kelly et al. 2014).

A physical understanding of GRBs and the conditions in which they form is thus necessary to put observations of highredshift GRBs into a cosmological context. Arguably, one of the key pieces to the puzzle of understanding GRB progenitors and their role in probing distant star formation lies in the nature of their host galaxies (e.g., Levesque 2014, for a review).

Seminal sample studies (e.g., Le Floc'h et al. 2003; Christensen et al. 2004; Tanvir et al. 2004; Levesque et al. 2010a) culminating in the compilation of GRB host properties of Savaglio et al. (2009) revealed a population of lowluminosity, low-mass, star-forming galaxies at $z \lesssim 1$. The host's UV emission is highly concentrated around the GRB position and their morphologies appear more irregular than the hosts of core-collapse supernovae (Fruchter et al. 2006; Svensson et al. 2010).

In line with this picture were Ly $\alpha$ properties (Fynbo et al. 2003; Milvang-Jensen et al. 2012) and early measurements of sub-solar GRB host metallicities (e.g., Prochaska et al. 2004; Gorosabel et al. 2005; Sollerman et al. 2005). These data supported a strong dependence of GRB formation with metallicity (e.g., Hirschi et al. 2005; Yoon et al. 2006) as would be theoretically expected in the collapsar model (Woosley 1993; MacFadyen \& Woosley 1999).

More recently, however, this previously quite uniform picture of GRB hosts became somewhat more diverse: the offset of GRB-selected galaxies towards lower metallicities in the massmetallicity relation (Levesque et al. 2010b) could, for example, be partially explained with the dependence of the metallicity of star-forming galaxies on SFR (e.g., Mannucci et al. 2011; Kocevski \& West 2011). Additionally, several metal-rich GRB hosts were discovered (Levesque et al. 2010c; Elliott et al. 2013; Schady et al. 2015), and extensive observation in multiband photometry revealed a population of red, high-mass, highluminosity hosts, mostly associated with dust-extinguished afterglows (Krühler et al. 2011; Rossi et al. 2012; Hjorth et al. 2012; Perley et al. 2013b). Similar GRBs were underrepresented in previous studies, illustrating the need for dust-independent, X-ray-selected samples such as The Optically Unbiased GRB Host survey (TOUGH, Hjorth et al. 2012) or the Swift GRB Host Galaxy Legacy Survey (SHOALS, Perley et al. 2015a).

Because of these inherent uncertainties from sample selection and small number statistics, the question of how directly GRBs trace star formation, and how representatively they select star-forming galaxies remains a matter of debate (e.g., Michałowski et al. 2012; Vergani et al. 2015; Schulze et al. 2015): a metal-dependence on the GRB selection is shown by e.g., Graham \& Fruchter (2013) or Perley et al. (2013b), while Hunt et al. (2014) advocate no strong evidence for GRBs to provide a biased census of star formation.

Elucidating the tight connection between star formation and GRBs also holds the promise of shedding more light on the nature of star-forming galaxies in general, especially at faint luminosities as well as their evolution at high redshift. Detailed spectroscopic observation of GRB hosts, however, remained challenging, in particular at $z>1$ : prominent tracers of the physical conditions in the hot gas are redshifted into the NIR where spectroscopy traditionally is much less efficient. Spectroscopic data for $z>1$ GRB hosts from emission lines is therefore available for only a handful of cases (e.g., Chen 2012; Friis et al. 2015; Piranomonte et al. 2015), and even at $z<1$ the largest samples (Savaglio et al. 2009; Levesque et al. 2010a; Graham \& Fruchter 2013) contain only 10-15 events with detailed information on the host's gas properties.

Here, we present initial results from emission-line spectroscopy of 96 GRB-selected galaxies in the redshift range $0.1<z<3.6$, with the bulk of the targets $(76 \%)$ between $0.5<z<2.5$. In this work, we focus on strong recombination and nebular lines such as the Balmer series and the forbidden transitions of [O II] or [O III]. The Ly $\alpha$ emission properties of the sample, a stacking analysis, and photometric follow-up observations will be discussed in detail elsewhere.

Throughout the paper, we report line fluxes in units of $10^{-17} \mathrm{erg} \mathrm{cm}^{-2} \mathrm{~s}^{-1}$, magnitudes in the $\mathrm{AB}$ system, wavelengths and redshifts in vacuum and a heliocentric reference frame, and errors at $1 \sigma$ confidence levels. We assume concordance cosmology (Planck Collaboration XVI 2014, $\Omega_{\mathrm{m}}=0.315, \Omega_{\Lambda}=0.685$, $\left.H_{0}=67.3 \mathrm{~km} \mathrm{~s}^{-1} \mathrm{Mpc}^{-1}\right)$, and a solar oxygen abundance of $12+\log (\mathrm{O} / \mathrm{H})=8.69$ (Asplund et al. 2009).

\section{Sample and observations}

\subsection{Targets}

To assemble our target list, we rely heavily on previous catalogs, in particular TOUGH, BAT6 (Salvaterra et al. 2012), from GROND (Greiner et al. 2011; Krühler et al. 2011), and SHOALS for which we retrieve X-Shooter ${ }^{1}$ (Vernet et al. 2011) optical/NIR spectroscopy from the ESO archive (PIs: de Ugarte Postigo, Flores, Fynbo, Kaper, Krühler, Malesani, Piranomonte, Rossi, Schady, Schulze). In addition, we also use GRB afterglow observations (PI: Fynbo) obtained with X-Shooter in target-ofopportunity mode.

We immediately removed events from the analysis that belong to the class of short GRBs such as GRB 130603B (e.g., de Ugarte Postigo et al. 2014). We include the host of GRB 100816A even though it cannot be uniquely attributed to either long or short category (Oates et al. 2010). To maintain a clean sample selected through genuinely long GRBs, this borderline case is not used in the discussion of galaxy properties in Sect. 5. GRB 100316D at $z=0.0592$ (e.g., Starling et al. 2011; Bufano et al. 2012) is the closest GRB in the sample. As the $\mathrm{X}$-Shooter slit covers only a small fraction of the host light, we cannot infer galaxy-integrated properties. We therefore also omit the GRB 100316D host galaxy in Sect. 5.

After an initial reduction and screening of the afterglow or host spectra, we excluded those events for which neither emission lines nor the stellar continuum of the host is detected: in the absence of a robust systemic redshift or in the presence of a very bright afterglow, no meaningful limits on emission-line fluxes can be established. We removed a total of nine host spectra from the sample in this step, primarily targeting very faint galaxies with $R \gtrsim 25.5 \mathrm{mag}$ (GRBs 050406, 060923A, 060923C, 060919, 090926A, 101219B, 110709B). Two excluded spectra

1 X-Shooter is a medium-resolution, cross-dispersed echelle spectrograph mounted at ESO's Very Large Telescope and sensitive from the atmospheric UV cutoff $(\sim 3000 \AA)$ up to the $K$-band. 
are likely those of foreground objects (i.e., misidentifications, GRBs 070808, 081210). Out of these nine host spectra, two have accurate afterglow redshifts (GRBs 090926A at $z=2.11$, 101219B at $z=0.55$ ). GRB 060923A has a photo- $z$ of $z \sim 2.6$ (Tanvir et al. 2008; Perley et al. 2013b).

The total number of GRB host galaxy spectra presented here is 96 , and nebular lines are detected for 91 of them. This sample advances in two crucial aspects from previous works: For the first time, it extends significantly beyond redshift of order unity owing to the sensitivity of X-Shooter up to $2.5 \mu \mathrm{m}$. Our observations thus cover prominent tracers ( $\mathrm{H} \alpha$ and $[\mathrm{N} \mathrm{II}]$ ) of the ionized gas up to $z \sim 2.5$, and [O II], $\mathrm{H} \beta$, and [O III] up to $z \sim 3.5$. Secondly, number statistics are approximately a factor five larger than in previous spectroscopic samples. A log summarizing the $\mathrm{X}$-Shooter observations is provided in Table 1.

\subsection{The sample}

The presented X-Shooter observations were selected from the ESO archive, obtained by several groups and programs over the course of several years (2009-2015), and are hence diverse. They can however be divided into two main sub-categories.

First, dedicated host spectroscopy, which aims on either measuring redshifts of GRBs for sample studies (e.g., Krühler et al. 2012b; Salvaterra et al. 2012) or the physical properties of individual GRB hosts (e.g., Vergani et al. 2011; Krühler et al. 2012a). Second, we also discuss GRB afterglow observations in which host emission lines are detected above the afterglow continuum.

Our GRB hosts are faint ( $21 \mathrm{mag} \lesssim R \lesssim 26$ mag with only a few exceptions) and their redshift distribution is broad extending up to $z \sim 3.5$. It has a median of $z_{\text {med }}=1.56$ and $35 \%$ of the targets are at $0.5<z<1.5$, and another $41 \%$ between $1.5<z<2.5$ (Fig. 1). It is hence shifted significantly towards lower redshifts when compared against GRB (afterglow) redshift distributions (Greiner et al. 2011; Jakobsson et al. 2012; Salvaterra et al. 2012; Perley et al. 2015a), an obvious result of a selection bias favoring $z<3.5$ galaxies for host spectroscopy. The brightness distribution of the galaxies peaks at around $R \sim 25$ mag with a median value of $R_{\text {med }} \sim 24.3 \mathrm{mag}$. The basic properties of our galaxy sample, optical brightness and redshift are shown in Fig. 1.

\subsection{Selection effects}

Galaxy samples obtained from GRBs are different than those from flux-limited surveys. Since the galaxy is localized by an external high-energy trigger - the GRB and its afterglow - the selection is initially independent of the galaxies dust content, brightness or SFR. If we studied the hosts of all (or a representative subset of) GRBs, we could examine their properties irrespective of any flux limit.

As we, however, heterogeneously draw our GRBs from a larger parent sample of Swift GRBs, it is important to test how representative our GRB hosts really are. This implies understanding the selection effects on the GRBs itself as well as those introduced through the follow-up observations. Dedicated spectroscopic host observations favor, for example, optically brighter galaxies on average: a precise slit alignment requires a prior detection via broad-band imaging (Sect. 2.3.2).

A further critical parameter is the amount of visual attenuation by dust along the GRB sight-line $A_{V}^{\mathrm{GRB}}$ (Krühler et al. 2011; Hjorth et al. 2012; Perley et al. 2013b, 2015b) because it

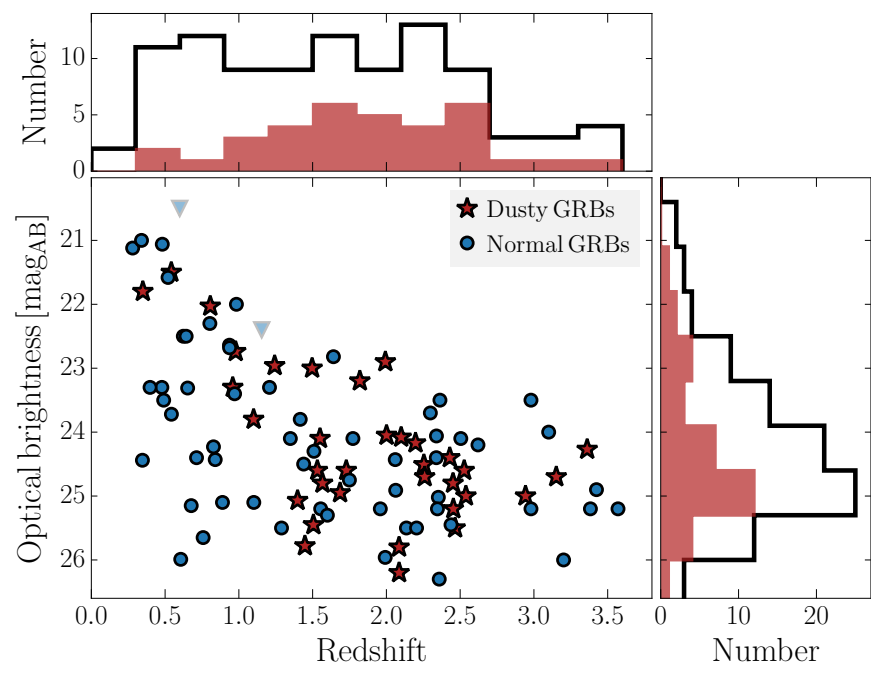

Fig. 1. Brightness and redshift distribution of the galaxies in our sample. Magnitudes are primarily measured in the $R / r$ filter ( $94 \%$ of the sample). For the remaining hosts, $V$ or $I / i$-band magnitudes are shown. Upper limits come from events for which the spectral continuum was dominated by a bright afterglow, they are denoted by downward triangles and are not included in the brightness histogram. Galaxies that hosted dusty GRBs (sightline $A_{V}^{\mathrm{GRB}}>1 \mathrm{mag}$ ) are indicated by red stars and red-filled histograms.

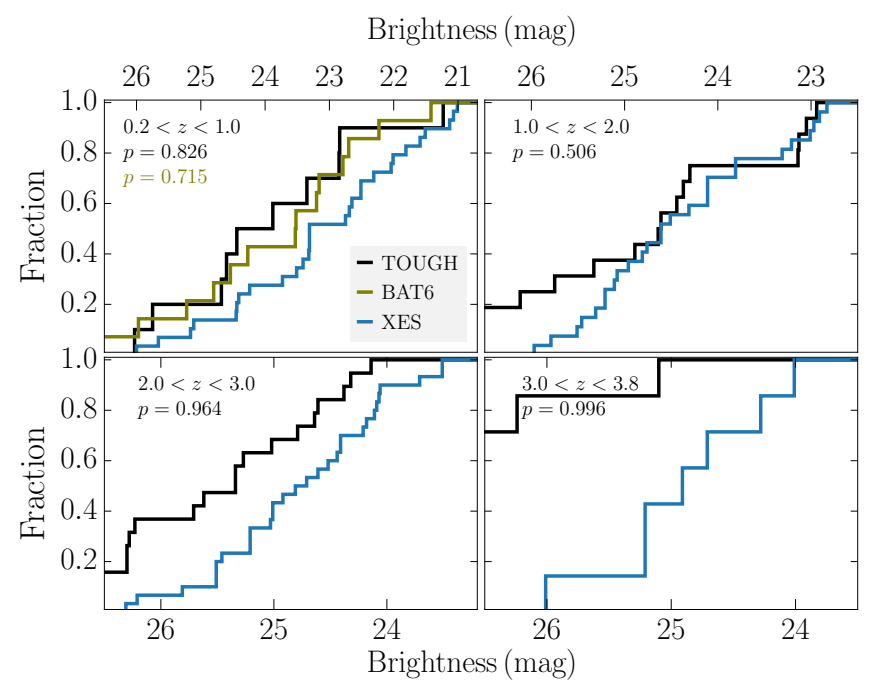

Fig. 2. Comparison of the cumulative brightness distributions of the host of our X-Shooter emission-line spectroscopy (XES) in blue against representative GRB host samples in black and olive lines (Hjorth et al. 2012; Vergani et al. 2015). In the top left of each panel, we show the K.S.-test $p$-value to reject the null-hypothesis that both samples are drawn from a similar parent distribution.

correlates with host properties - galaxies hosting dusty ${ }^{2}$ GRBs have an order of magnitude higher stellar mass, luminosity, and SFR.

The presence of GRBs with suppressed optical afterglows (e.g., Groot et al. 1998; Levan et al. 2006) constituted a

2 We prefer to work with dusty GRBs instead of dark GRBs. The definition of dark GRBs is not unique (Jakobsson et al. 2004; van der Horst et al. 2009), depends on the physics of the GRB shockwave and on the time and filter when the observations are performed (Greiner et al. 2011). The rest-frame dust column density along the GRB sight line $A_{V}^{\mathrm{GRB}}$ is thus a more physical quantity than the darkness of a afterglow. 
significant uncertainty in previous studies because they were underrepresented in redshift distributions (e.g., Fig. 9 in Hjorth et al. 2012). As X-Shooter is extremely efficient in providing galaxy redshifts (Krühler et al. 2012b), there is an above average fraction of dusty $\left(A_{V}^{\mathrm{GRB}}>1 \mathrm{mag}\right) \mathrm{GRBs}$ in this work: it is $35 \pm 5 \%$ here, but $20 \%$ to $30 \%$ in unbiased GRB samples (Greiner et al. 2011; Melandri et al. 2012; Littlejohns et al. 2015) in a similar redshift range (Sect. 2.3.3).

\subsubsection{Absolute statistics}

First, we examine absolute number statistics at $z<1$, where the sample of GRBs with redshifts is highly complete ${ }^{3}$. In total, we have targeted $\sim 50 \%$ (32) of all $z<1$ GRBs in the Swift-era. The remaining $50 \%$ were either too northern, too close to the Galactic plane, or not observable because of technical or telescope time limitations. From the initially targeted 32 galaxies at $z<1$, we detect emission lines for 30 (94\%). The only two non-detections are GRB 110715A $(z=0.82)$, which spectrum was dominated by a $R=18.5 \mathrm{mag}$ afterglow (Piranomonte et al. 2011), and GRB 101219B at $z=0.55$ (Sparre et al. 2011).

\subsubsection{Brightness distributions}

To test whether the pre-imaging introduces biases towards optically bright hosts, we compare the distribution of $R$-band magnitudes (Fig. 1) with those of two representative ${ }^{4}$ and redshiftcomplete samples (Hjorth et al. 2012; Vergani et al. 2015) which we use as a control group.

Figure 2 shows the respective cumulative brightness distributions in four redshifts intervals. At low redshift $(z<2)$, a K.S.-test returns no strong evidence that both samples draw from different parent distributions (K.S.-test $p$-values of 0.87 and 0.50 at $z<1$ and $1<z<2$, respectively) - except possibly a $\sim 20 \%$ fraction of very faint hosts in TOUGH at $1<z<2$ that is not present here. Above $z \sim 2$, however, a selection effect is clearly evident: our galaxies are substantially brighter than the reference sample (K.S.-test $p$-values of $p=0.96$ and $p=0.996$ at $2<z<3$ and $3<z<3.8$, respectively).

\subsubsection{The fraction of dusty GRBs}

Our $z<2$ galaxies therefore have a similar brightness distribution to unbiased GRB host samples. The over-proportionality of galaxies with dust-reddened afterglows in the sample, however, could nevertheless have an impact on their physical parameters.

As representative comparison sample of the dust content towards GRBs $\left(A_{V}^{\mathrm{GRB}}\right)$, we avail of the union of the afterglows from Greiner et al. (2011) and Covino et al. (2013). After excluding duplicates, and limiting the samples to the same redshifts as the host spectroscopy $(z<3.6), 75$ GRBs remain to constrain the

\footnotetext{
3 Because of the exquisite X-ray positions from Swift and extensive follow-up efforts from the ground with e.g., TOUGH, GROND, or SHOALS, low-redshift GRBs are very unlikely to be missed if they have favorable observing conditions.

4 Representative, or unbiased, samples fully exploit the advantages of the high-energy selection by GRBs. Since they are based on criteria that are not connected to the burst's physical environment, they do not depend on dust obscuration or galaxy luminosity. They allow us to study afterglows or hosts in a truly representative manner.
}

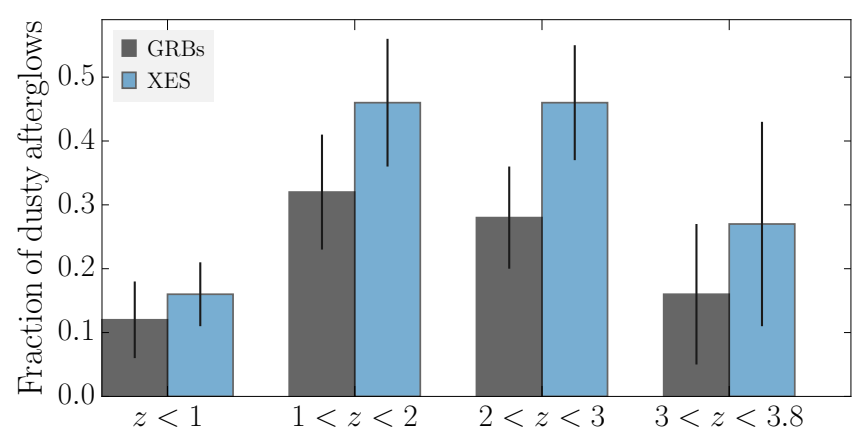

Fig. 3. Redshift dependent fractions with errors of dusty afterglows from our X-Shooter emission-line spectroscopy (XES) in blue against representative GRB samples in black (Greiner et al. 2011; Covino et al. 2013).

fraction of dusty ( $A_{V}^{\mathrm{GRB}}>1 \mathrm{mag}$ ) GRBs. Figure 3 compares these values with those of our GRBs ${ }^{5}$.

The ratio of dusty-to-normal afterglows is somewhat higher at $z \sim 2$ than at $z<1$ (Fig. 3). In addition, our sample has a slightly higher fraction of dusty afterglows at all redshifts than uniform GRB control samples. The data from galaxies selected through dusty GRBs are highlighted by stars in the following plots to illustrate their influence on correlations and GRB host evolution.

\subsection{A Stochastic analysis}

To correct for the over-proportionality of dusty GRBs, we statistically create samples that have the right fraction of dusty-tonormal afterglows as follows: we use the initial 96 hosts and randomly draw $10^{6}$ sub-samples while using the previously established dusty GRB fraction as a strict prior. Specifically, this means applying a redshift-dependent probability to prevent a galaxy which hosted a dusty GRB to enter in sub-samples.

The remaining analysis in this paper is performed with all $10^{6}$ sub-samples where each is a random representation of the input catalog but with a correct proportionality between dusty and normal GRBs. Nebular line fluxes are described by a Gaussian probability distribution with mean and standard deviation given by the raw measurement and error. Since the likelihood of keeping dusty GRBs has an error itself, we propagate in this way not only the statistical uncertainty due to sample size and the measurement error, but implicitly also the uncertainty in the prior, i.e., the dusty GRB fraction $(28 \pm 8 \%$ at $2<z<3$, for example).

The result of this procedure are $10^{6}$ different distributions of host parameters, correlations, and best-fit values. In the following, we then provide for all parameters or correlations the median of the a-posteriori probability and the $1 \sigma$-equivalent range as error to represent their distribution.

Whenever we study GRB host parameters, we will plot two data sets: first, the more meaningful distribution restricted to $z<2$ (Sect. 2.3.2) and derived from the stochastic sample including error-bars always in blue. Second, we will also show the

5 Taken from the literature (Cenko et al. 2009; Krühler et al. 2011; Zafar et al. 2011; Perley et al. 2015a) or derived from our own afterglow data. We assign GRBs to dusty afterglows in cases where we can constrain $A_{V}^{\mathrm{GRB}}>1 \mathrm{mag}$ or when the optical/NIR afterglow is significantly underluminous with respect to the X-ray data. For example, GRB 120119A is dusty, although it had a bright optical counterpart (Morgan et al. 2014), but GRB 100606A is normal (not-dusty), even though it did not (Nicuesa et al. 2010). 


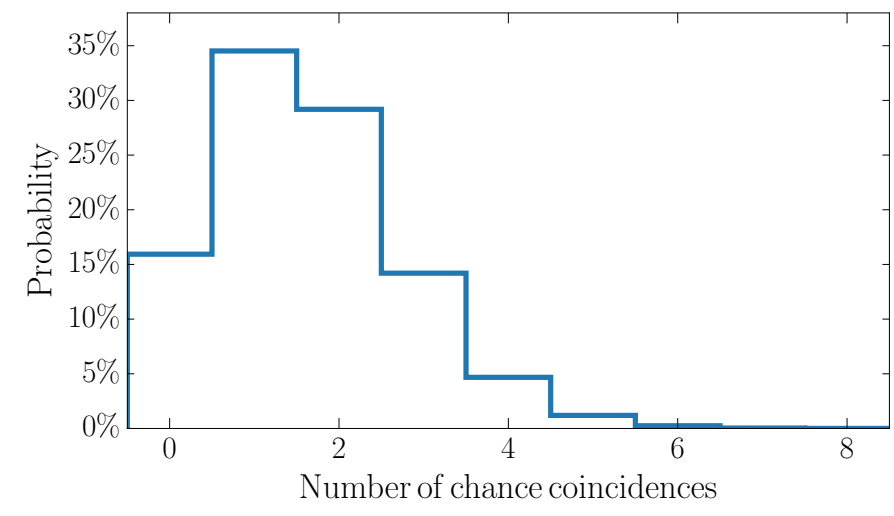

Fig. 4. Probability distribution of chance coincidence, or the likelihood of having a given number of galaxies in the total sample wrongly associated with the GRB. The probability that we have misidentified a total of 2 out of 96 galaxies is, for example, $30 \%$.

histogram of all measured values in black to illustrate the impact of the sample cuts. At $z>2$, we show the resulting parameters and distributions (without error regions) as reference. The selection effect towards optically bright galaxies at $z>2$, however, will often prevent us from deriving robust conclusions in this redshift range.

\subsection{Misidentification of hosts}

We use standard procedures (Bloom et al. 2002; Perley et al. 2009) to assign galaxies to GRBs by using the afterglow position with the highest accuracy available. A crucial basis of this work are accurate GRB positions which are routinely available only since the launch of Swift (Gehrels et al. 2004; Burrows et al. 2005).

In the best cases (16 of 96 GRBs), an afterglow redshift is set by fine-structure lines (e.g., Vreeswijk et al. 2007), and a matching emission line redshift as well as a sub-arcsecond errorcircle leave hardly any room for ambiguity. In total, 73 of the GRBs have localizations with an accuracy of around 0.'5 through radio, optical or Chandra X-ray afterglow observations.

In the remaining cases (23 of 96 GRBs), the host association relies on the X-ray position from Swift which constrains the GRB position to a circle with $\sim 1$ '. 5 radius at $90 \%$ confidence. The probability of misidentification, i.e., the galaxy that we study is not related to the GRB of interest is then a function of positional accuracy, galaxy brightness, and density of galaxies for the given galaxy type, brightness, and coordinates (e.g., Rossi et al. 2014). For average values of galaxy densities in empty fields (Capak et al. 2004; Gawiser et al. 2006), positional errors of $11^{\prime \prime} 5$, and galaxy brightnesses of $r \sim 25$ th mag, the chance of finding a random galaxy in the error circle is around $1-2 \%$.

To quantify the total number of chance coincidences, or misidentifications in the full sample, we use the GRB host brightnesses from Fig. 1 to assess how many similarly bright field galaxies (Capak et al. 2004; Gawiser et al. 2006) are statistically expected within an area corresponding to the GRB's localization accuracy. After excluding those events for which the afterglow fine-structure/host redshift match, we calculate the probabilities for a given number of misidentifications through a Monte-Carlo method with $10^{6}$ trials. The resulting distribution is shown in Fig. 4, and has a peak at around 1-2 events, with a tail out to a total of 5 misidentified galaxies. Very few field galaxies are thus interloping in our GRB host sample and their impact on the main conclusions will be limited.

\section{X-Shooter optical/NIR spectroscopy}

$\mathrm{X}$-Shooter operates in three dichroic-separated arms simultaneously: the ultra-violet, blue (UVB, 3000-5600 $\AA$ ), the visual (VIS, 5500-10 020 ̊), and the near-infrared (NIR, $10000-$ $24800 \AA$ ) arm. Each of the three arms has its own slit, echelle grating, cross-disperser, and detector, and we refer to Vernet et al. (2011) for a detailed description of the instrument. The resolving power of X-Shooter depends on the arm and slit width and is typically between $R=4000$ and $R=10000$ for the used setups 6 . Unless dictated by other observational constraints such as nearby bright objects, the observations were obtained in a nodding pattern in multiples of an ABBA sequence. The nodlength is constrained by the short slit-length of X-Shooter (11.'0) and is usually $5{ }^{\prime \prime} 0$.

Whenever possible, slit loss due to atmospheric dispersion was minimized by observing at the parallactic angle and at minimum airmass. A large subset of the host observations were obtained before X-Shooter's ADC failure in August 2012 and most of the emission lines are located in the NIR part of the spectrum (e.g., the bluest of the lines of interest ([O II]) is already above $10000 \AA$ at $z>1.5$ ) where atmospheric dispersion is less severe. The lack of ADCs was not a primary concern in the analysis.

\subsection{Data reduction}

The basis of the data reduction was the X-Shooter pipeline supplied by ESO in its version 2 . 2 . 0 or higher (Goldoni et al. 2006; Modigliani et al. 2010), which we used for flat-fielding, order tracing, rectification, and initial wavelength calibration and flexure compensation via arc-lamp frames (also Sect. 3.2). These steps were applied to the individual frames of the individual arms of each set of spectra. During rectification, we chose a dispersion of $0.4 \AA / p x$ (UVB/VIS arm) and $0.6 \AA / p x$ (NIR arm). This minimizes correlated noise, while at the same time maintaining sufficient sampling for emission lines down to a velocity dispersion $\sigma$ of $\sim 20 \mathrm{~km} \mathrm{~s}^{-1}$.

We use our own software and algorithms for bad-pixel and cosmic ray detection, as well as sky-subtraction and stacking of individual exposures. We further corrected the inter-order background for an apparent problem in the X-Shooter pipeline. In the UVB and VIS arm, we estimate the sky background locally in a small region around the spectral trace. For the NIR data with its abundant and strong sky-lines and high background, the frames for each set of observation are grouped in pairs of two such that they were taken in a different sky-position but as close in time as possible. Each two frames were then subtracted from each other for the background estimation.

A typical observation (Table 1) consisting of four exposures in the UVB/VIS, and twelve in the NIR arm leads to four and six sky-subtracted, rectified, wavelength and flux-calibrated (Sect. 3.3) frames in the UVB/VIS and NIR arm, respectively. These single frames are then averaged using a weight function from the variance or the signal-to-noise ratio $(\mathrm{S} / \mathrm{N})$. Previously detected bad-pixels and cosmic rays are masked in the stacking process.

From the combined two-dimensional frame we then optimally extract the one-dimensional spectrum. The weight

\footnotetext{
6 https://www . eso.org/sci/facilities/paranal/ instruments/xshooter/inst.html
} 
function of the extraction is derived using the collapsed profile of either the emission lines themselves or the spectral continuum in case it is detected at a sufficient $\mathrm{S} / \mathrm{N}$ level.

\subsection{Wavelength calibration}

$\mathrm{X}$-Shooter is mounted on the VLT Cassegrain focus, so it suffers from instrument flexures and misalignments of the wavelength solution between day-time calibration and night-time science observations. We correct for these flexures first by using the ESO pipeline and frames supplied by X-Shooter's active flexure compensation. In a second step, we use a theoretical emission spectrum of the Paranal sky (Noll et al. 2012). In the VIS and NIR arm, we divide the spectrum into 10-15 slices, and cross-correlate the observed and theoretical sky-spectrum. This provides wavelength offsets averaged over the respective wavelength interval. A low-order polynomial was then fitted to the data, and the respective wavelength offset as derived from the fit was applied to the wavelength scale. Typical offsets are smaller than around $0.4 \AA$ in the NIR arm and $<0.1 \AA$ in the UVB/VIS. Because of the lack of strong sky features in the UVB arm blueward of $5000 \AA$, we apply only a constant offset to the wavelength scale in the UVB arm.

\subsection{Flux calibration}

Correct flux scales of the emission line spectra are fundamental because they have a strong impact on the measurement of global galaxy characteristics. We first flux-calibrated each of the individual spectra with the nightly standard star taken with a slitwidth of 5.'0. Standard stars are different for each observation, and the full set is given at the ESO webpages ${ }^{7}$. The resulting response curves were thoroughly validated against each other. To limit the effect of a time-dependent illumination by a timevarying flat-field lamp, particularly evident in the red part of the NIR arm, we used the same flat field for science and standard frames to correct for pixel-to-pixel variations.

Even with a well-defined instrumental response, the finite slit width inevitably leads to slit-loss. Slit-loss depends primarily on the ratio between slit-width and the convolution of the pointspread function delivered by the telescope with the spatial extent of the sources on the sky. In addition, the sky transparency varied significantly during the course of some of the observations due to clouds, leading to an additional decrease in flux compared to the standard star observation.

Accurate broadband photometry, however, is available to us providing means to estimate galaxy-integrated line-fluxes. The ratio between photometry and a synthetic brightness of the spectrum integrated over the band-pass of the respective filter yields a scale factor to estimate slit-loss (which we call $\tau$ ). In the ideal case, multiple independent photometric bands in each of $\mathrm{X}$-Shooter's arms allow us to corroborate and check the intraand inter-arm flux calibration. For many hosts, this is not the case and we assume that $\tau$ is constant in each arm. We then multiply the line flux by $\tau$ and propagate errors accordingly. $\tau$ is in the range between 1 and 3, and its distribution has a median and $1 \sigma$ bounds of $\tau_{\text {med }}=1.6_{-0.5}^{+0.7}$.

Deriving $\tau$ for the individual arms is only possible for a bright continuum ( $r<23 \mathrm{mag}$ ), i.e., luminous hosts or afterglows. For most of the fainter hosts $(r \sim 25-26 \mathrm{mag})$, the continuum is detected in the bluer arms only leading to relatively

\footnotetext{
7 http://wWW.eso.org/sci/facilities/paranal/

instruments $\backslash$ /xshooter/tools/specphot_list.html
}

large errors in $\tau$ and the line fluxes. In cases where no continuum is detected, or no comparison photometry is available in the given arm, we assume that $\tau$ is similar between all arms. In cases where we can measure $\tau$ for different arms or wavelengths, it is consistent within errors. This provides confidence that the scaling procedure is reliable within the quoted error bars.

Errors in $\tau$ come from the photon noise of the spectral continuum, the accuracy of the comparison photometry, and the scatter of different filters in each arm if available. Line ratios of adjacent lines (such as $\mathrm{H} \alpha$ and $[\mathrm{N}$ II] or the [O III] doublet and $\mathrm{H} \beta$ ) and the physical properties that they are proxies for, have the same scaling factor in all cases. We associate the line-flux error from photon statistics with a statistical error, and the one derived from the scaling factor with a systematic one, and provide full details of both errors in the line-flux tables (Tables 2 and 3). All fluxes, spectra, and photometry are corrected for the Galactic foreground reddening according to Schlafly \& Finkbeiner (2011) assuming $R_{V}=3.08$ (Pei 1992).

\subsection{Flux limits and sensitivities}

To better understand the fundamental properties and constraints of our observations, we look closer at the sensitivity limits. From a typical observational setup with an integration time of $4 \times 600 \mathrm{~s}$, we derive the noise characteristics of the spectrum. Artificial emission lines of $\mathrm{H} \alpha$ and the [O II] doublet at various redshifts with a typical intrinsic line width of $\sigma=70 \mathrm{~km} \mathrm{~s}^{-1}$ (Sect. 5.5) are then added to the noise spectrum.

We record the emission-line flux that would have been detected with a combined significance of at least $6 \sigma$ over the noise level in the one-dimensional spectrum. The significance level is set conservatively because we have no a priori knowledge of the redshift in many cases ${ }^{8}$. Line-flux sensitivities for $\mathrm{H} \alpha$ and [O II] are then converted into SFRs using the methods from Sect. 4.4.

Figure 5 illustrates the redshift dependence of the detectable SFR. At $z<0.5, \mathrm{H} \alpha$ lies in the visual arm of X-Shooter with its sensitive CCD and low sky-background. The SFR limit is a few $10^{-4} M_{\odot} \mathrm{yr}^{-1}$ at $z \sim 0.1$ and increases to $8 \times 10^{-3} M_{\odot} \mathrm{yr}^{-1}$ at $z \sim 0.5$. In the non-extinguished case (solid lines in Fig. 5), the SFR sensitivity stays below $1 M_{\odot} \mathrm{yr}^{-1}$ up to $z \sim 1.7$.

Above $z \sim 2.5$, the sensitivity to unextinguished SFR is driven by $[\mathrm{O}$ II $]$ and stays at around a few $M_{\odot} \mathrm{yr}^{-1}$ up to $z \sim 3.7$ above which it rises beyond $10 M_{\odot} \mathrm{yr}^{-1}$. Adding dust increases these limits considerably, in particular for [O II] (dashed lines in Fig. 5).

Noteworthy are redshift ranges where the SF-tracers are located in regions of prominent telluric absorption bands (black lines in the top panel of Fig. 5). In a small window around $z \sim 1$ and redshift ranges of $1.7<z<2.0,2.6<z<3.0$, and $z>3.7$, atmospheric absorption makes the detection of emission lines challenging from the ground.

\subsection{Auxiliary photometry}

For most of the targets the photometry used to flux-calibrate the spectra is either provided by TOUGH (Hjorth et al. 2012), or the literature referenced in Table 1 . If not available elsewhere,

\footnotetext{
8 The look-elsewhere effect: It is for example very likely to find $3 \sigma$ noise peaks in the medium-resolution spectrum of X-Shooter just because the parameter space to be searched is so large. If the redshift is known, the locations of the emission lines are fixed, and the probability of finding significant noise peaks by chance in the correct wavelength range drops substantially.
} 


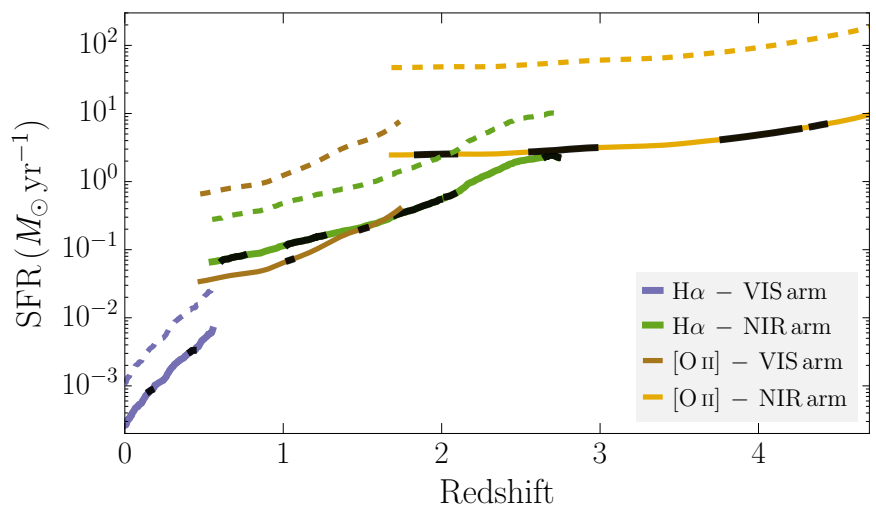

Fig. 5. Colored lines represent the sensitivity for $\mathrm{H} \alpha$ and $\left[\mathrm{O}_{\mathrm{II}}\right]$ converted into a SFR. Solid/dashed lines denote the case of $A_{V}=0 \mathrm{mag}$ and $A_{V}=2 \mathrm{mag}$, respectively. Black lines indicate redshift ranges where the line is located in windows of negligible atmospheric transmission. Skylines result in high SFR limits on small scales, but have been omitted to enhance clarity.

dedicated observations were performed with GROND (Greiner et al. 2008) at the 2.2 m MPG telescope on LaSilla, HAWK-I (Kissler-Patig et al. 2008), FORS2 (Appenzeller et al. 1998), LRIS at Keck (Oke et al. 1995) or ALFOSC at the $2.5 \mathrm{~m}$ Nordic Optical Telescope (NOT).

The optical/NIR imaging data were reduced in a standard fashion using dedicated routines in pyraf/IRAF (Tody 1993) in the case of GROND, VLT, and NOT photometry or IDL for Keck/LRIS. Data reduction and photometry follow the procedures established in Krühler et al. (2008) in the former case and is taken from the Keck GRB Host Survey (Perley et al. 2013a) in the latter case. The photometric solution of each field was tied to the SDSS survey for those GRB fields which were covered by SDSS DR8 (Aihara et al. 2011), zero-points derived from the observation of standard stars, or our own set of secondary calibrators in the field. Typically, secondary calibrators were again derived from observing SDSS fields close in time to the GRB observations.

\section{Analysis and results}

\subsection{Line flux measurements}

Fluxes of emission lines were measured by fitting a superposition of Gaussian functions to the data, with the continuum set in small $( \pm 40 \AA)$ regions around the wavelength of interest free of emission-, telluric-, and sky-lines. In most cases, a single Gaussian and constant continuum describes the line with sufficient accuracy (Fig. 6). We also measured the line-flux through numerical integration, and its result was used in cases where the lines show clear velocity structure.

For weak emission lines such as [Ne III] or [N II], we tied the Gaussian centroid (set by the galaxy redshift) and the line width to those of strong lines.

Finally, a synthetic Paranal sky radiance and transmission spectrum (Noll et al. 2012) was used to flag regions of telluric absorption and sky emission, and affected data were automatically discarded from the line fit (Fig. 6). The results of the line flux measurements are given in Tables 2 and 3.

Many of the emission lines are not detected at high signal-tonoise ratios $(\mathrm{S} / \mathrm{N})$. If the spectral range is covered by $\mathrm{X}$-Shooter, we decided to quote the actual measurement instead of providing an upper limit to maximize the information on e.g., line ratios under the given photon statistics.

We have not corrected the Balmer line fluxes for the underlying Balmer stellar absorption even though we note that it can have a measurable influence on the line fluxes and thus the derived physical properties of the emitting gas (Wiersema 2011). The $\mathrm{S} / \mathrm{N}$ of the emission lines and stellar continuum is not high enough to resolve and fit the absorption component separately. Generally, the Balmer absorption correction is small for galaxies with masses below $10^{10} M_{\odot}$.

At $z \sim 0.8$, for example, it has an equivalent width $(\mathrm{EW})$ of $1 \AA$ for galaxies of $10^{10} M_{\odot}$ at $\mathrm{H} \beta$ (Zahid et al. 2011). The $\mathrm{EW}$ of the $\mathrm{H} \alpha$ line is high enough $\left(E W_{\text {rest }}>20 \AA\right)$ to make the correction negligible, but it can have an effect on the other Balmer line-fluxes. We estimate $\lesssim 5 \%$ for $\mathrm{H} \beta$ on average, well below the average statistical error of the line flux measurements (Table 2).

In cases where [O III] $\lambda 5007$ is not well constrained by the data, but [O III] $\lambda 4959$ is, or vice versa, we assume that $f_{[\mathrm{OIII}(\lambda 4959)]}=1 / 3 \cdot f_{[\mathrm{OIII}(\lambda 5007)]}($ Storey \& Zeippen 2000).

\subsection{Emission-line widths}

We measure the intrinsic line-width $(\sigma)$ of the hot gas using nebular lines of highest $\mathrm{S} / \mathrm{N}$, usually $\mathrm{H} \alpha$, or [O III]. If the line-shape is adequately represented with a Gaussian function, we use the instrumental resolution $\Delta V$ and subtract it quadratically from the fitted FWHM, giving $\sigma$ as $\sigma=\sqrt{F W H M^{2}-\Delta V^{2}} /(2 \sqrt{2 \ln 2})$. If the line-shape is clearly asymmetric, we directly measure FWHM in the data. Errors on $\sigma$ are derived via the errorspectrum and propagated accordingly.

The physical line-width is well-resolved through the medium resolution of X-Shooter (typically $\Delta V \sim 35 \mathrm{~km} \mathrm{~s}^{-1}$ in the visual arm, or $\Delta V \sim 50 \mathrm{~km} \mathrm{~s}^{-1}$ in the NIR arm). The line-width measurements are provided in Table 4.

\subsection{New GRB redshifts}

To derive GRB redshifts from host galaxy data (Table 5), we use both the emission lines as detailed in Krühler et al. (2012b), spectral breaks in the stellar continuum (Jakobsson et al. 2012) as well as standard photo- $z$ techniques in LePhare (Arnouts et al. 1999; Ilbert et al. 2006) from supplementary multi-band photometry. For galaxies with more than one detected emission line, the redshift solution is accurate, unique, and robust. For the other, somewhat more ambiguous cases we provide more information in Appendix A.

In particular, we provide redshifts for six GRBs (GRB 050714B at $z=2.438$, GRB $060306^{9}$ at $z=1.56$ GRB $060805 \mathrm{~A}^{10}$ at $z=2.363$, GRB 060923B at $z=1.509$, GRB 070224 at $z=1.992$, and GRB 070328 at $z=2.063$ ) in the TOUGH sample (Hjorth et al. 2012). This brings the redshift completeness of TOUGH to $87 \%$ (60 out of 69 events), with a median/average redshift of $z_{\text {med }}=2.10 \pm 0.18$ and

9 The redshift of GRB 060306 has been discussed in Jakobsson et al. (2012) and Perley et al. (2013b), already suggesting $z=1.55$ as the likely redshift based on a VLT/FORS spectrum in the former, and extensive photometry in the latter case. The detection of multiple emission lines unambiguously confirms $z=1.56$ as the correct redshift.

10 The association of GRB 060805A is ambiguous because two galaxies are detected in the Swift XRT error-circle. Here, we provide the redshift for the fainter object. The brighter object has a redshift of $z=0.603$ (Jakobsson et al. 2012). 


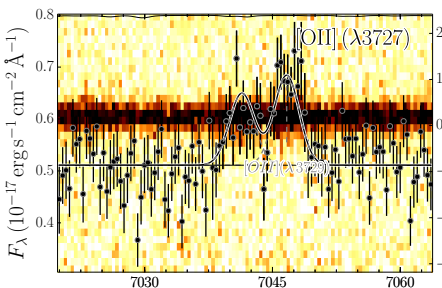

Observed wavelength $(\AA)$

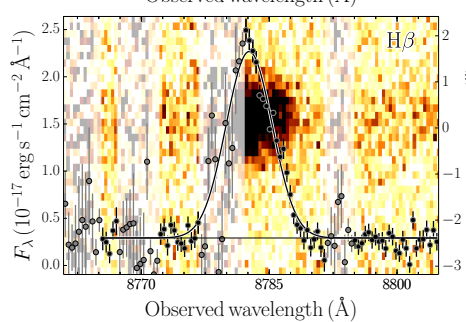

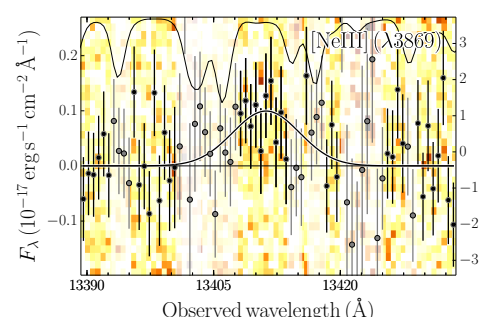

Observed wavelength $(\AA)$
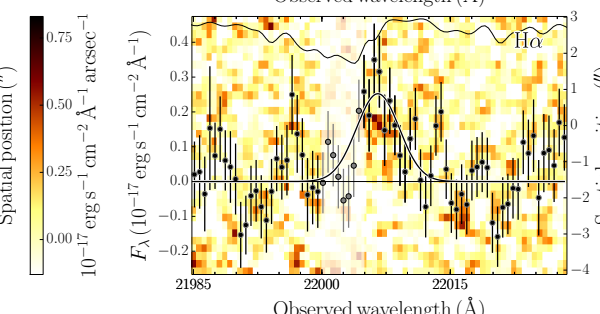
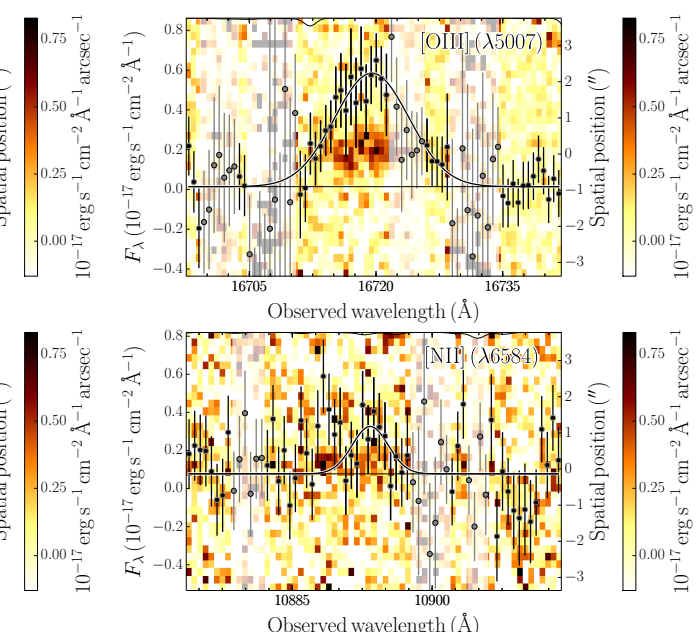

Fig. 6. Examples of line-flux measurements for nebular lines at different signal-to-noise level. In each panel, the background is the two-dimensional spectrum, where the spatial direction is plotted against the right $y$-axis. The color coding is indicated by the color bar. The one-dimensional spectrum with a Gaussian fit are shown in black points and line. Gray data points are excluded from the fit because of telluric or skyline contamination. A telluric transmission spectrum is plotted in a thin black line at the very top of each panel. From top left to bottom right: [O II] (GRB 140506A, with afterglow background), [Ne III] (GRB 100424A), [O III] (GRB 070129), H $\beta$ (GRB 051022), H $\alpha$ (GRB 070110), [N II] (GRB 050416A).

Table 5. New GRB redshifts.

\begin{tabular}{|c|c|c|}
\hline GRB host & Redshift & Spectral features \\
\hline GRB 050714B & 2.4383 & {$[\mathrm{O}$ III $](5007), \mathrm{H} \alpha$} \\
\hline GRB $060306^{a}$ & $1.5585 / 1.5597$ & {$[\mathrm{O}$ II $], \mathrm{H} \alpha$} \\
\hline GRB $060805 A^{b}$ & 2.3633 & $\mathrm{H} \beta,[\mathrm{O} \mathrm{III}](5007), \mathrm{H} \alpha$ \\
\hline GRB 060923B & 1.5094 & {$[\mathrm{O}$ III $](5007), \mathrm{H} \alpha$} \\
\hline GRB 070224 & 1.9922 & [O III] $(4959,5007)$ \\
\hline GRB 070328 & 2.0627 & {$[\mathrm{O} \mathrm{II}], \mathrm{H} \beta,[\mathrm{O} \mathrm{III}]$} \\
\hline GRB 070521 & 2.0865 & $\mathrm{H} \alpha$ \\
\hline GRB 080602 & 1.8204 & [O II], [O III], $\mathrm{H} \alpha$ \\
\hline GRB 090201 & 2.1000 & {$[\mathrm{O} \mathrm{II}], \mathrm{H} \beta,[\mathrm{O} \mathrm{III}], \mathrm{H} \alpha$} \\
\hline GRB 100508A & 0.5201 & [O II $], \mathrm{H} \beta,[\mathrm{O} \mathrm{III}], \mathrm{H} \alpha$ \\
\hline GRB 111129A & 1.0796 & [O II $]$ \\
\hline GRB 120211A & $2.4 \pm 0.1$ & Ly- $\alpha$ break \\
\hline GRB 120224A & $1.1 \pm 0.2$ & Balmer break \\
\hline GRB $120805 A$ & $3.1 \pm 0.1$ & Ly- $\alpha$ break \\
\hline GRB 121209A & $2.1 \pm 0.3$ & Balmer break \\
\hline GRB 140114A & $3.0 \pm 0.1$ & Ly- $\alpha$ break \\
\hline
\end{tabular}

Notes. The horizontal lines denote the separation between TOUGH GRBs (upper part), BAT6 (middle part) and others. ${ }^{(a)}$ Emission lines of the host of GRB 060306 have a double-peaked profile, and we thus provide two redshifts. ${ }^{(b)}$ Ambiguous association because two galaxies are present in the XRT error-circle. This is the redshift for the fainter of the two objects, for details see Jakobsson et al. (2012).

$z_{\mathrm{avg}}=2.20 \pm 0.17$, respectively. GRB 070328, GRB 070521 $(z=2.087)$, GRB $080602(z=1.820)$, and GRB 090201 $z=2.100$ are part of BAT6, so its completeness is now $97 \%$ (56 out of 58 events) with $z_{\text {med }}=1.67 \pm 0.15$ and $z_{\mathrm{avg}}=1.75 \pm 0.13$.

In addition, we measure or constrain the redshifts of seven GRBs based on emission lines or spectral breaks in the stellar continuum (Table 5).

\subsection{Scaling relations for star formation rates}

Since $\mathrm{H} \alpha$, the most reliable tracer of star formation in the rest-frame optical wavelength range, is not visible in all of

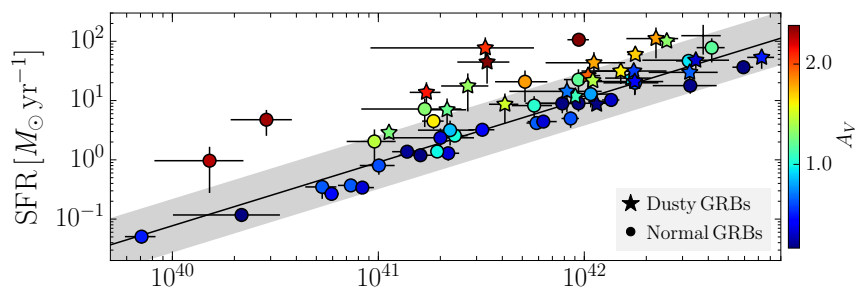

Uncorrected [OII] luminosity [ $\left.\operatorname{erg~s}^{-1}\right]$

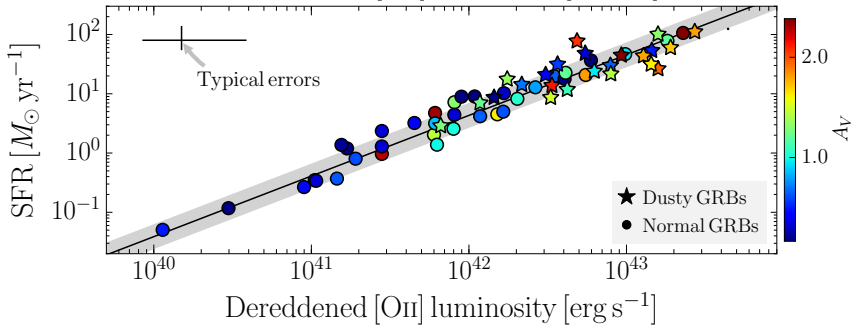

Fig. 7. Scaling relations between emission-line luminosity and SFR. Uncorrected/de-reddened [OII] luminosity against SFR in the upper and lower panel, respectively. Error-bars in the lower panel are omitted to enhance clarity (typical errors in the top left corner). The colorcoding of the individual measurements corresponds to $A_{V}$ as indicated by the color bar. The solid black lines show the best-fit scaling relations from Eq. (1). The gray-shaded area denotes the $1 \sigma$ scatter in the relation -0.45 dex in the upper and 0.19 dex in the lower panel.

the spectra, we use our sample to derive scaling relations between the luminosity of other strong nebular lines and the dustcorrected $\mathrm{SFR}_{\mathrm{H} \alpha}$. To establish the comparison sample, we avail of those galaxies for which both $\mathrm{H} \alpha$ and $E_{B-V}$ from the Balmer decrement (Sect. 5.3) can be constrained. We convert the dustcorrected $\mathrm{H} \alpha$ luminosity into a SFR based on the widely used Kennicutt (1998) relation adopting a Chabrier (2003) IMF. This provides us with a reference sample with reddening-corrected SFRs based on $\mathrm{H} \alpha$.

We then plot other strong emission lines against SFR (e.g., Fig. 7) and minimize the scatter of the data against scaling relations in the form of $\log \left(S F R_{\mathrm{H} \alpha}\right)=a+b \cdot \log \left(L_{42}\right)$. Here, $10^{a}$ is the SFR in $M_{\odot} \mathrm{yr}^{-1}$ for a benchmark line luminosity of $L_{42}=10^{42} \mathrm{erg} \mathrm{s}^{-1}$, while $b$ takes into account a possible 
evolution between SFR and line flux with galaxy properties. These scaling relations then constrain the SFR for hosts for which other nebular lines than $\mathrm{H} \alpha$ are available.

A slope of $b=1$ provides a direct proportionality between e.g., [O II] luminosity and SFR (Kennicutt 1998; Savaglio et al. 2009). $b$ significantly different from unity indicates that the relation between SFR and line flux changes with galaxy properties, for instance metallicities, dust attenuation or ionization (e.g., Kewley et al. 2004). One would thus ideally add a further dependence in the relation based on e.g., metallicity, stellar mass or luminosity for accurate SFRs (Kewley et al. 2004; Gilbank et al. 2010). In the absence of further information on the galaxy apart from line flux, however, all the physical changes in the SFR to line-flux relation are implicit in the empirical factor $b$.

[O II] for example, is strongly correlated with SFR (Fig. 7) and based on 60 hosts at $0.3<z<2.5$ we derive

$$
\begin{aligned}
& \log \left(S F R_{\mathrm{H} \alpha}\right)=1.02+1.07 \cdot \log \left(L[\mathrm{O} \text { II }]_{\text {,uncor, } 42}\right) \\
& \log \left(S F R_{\mathrm{H} \alpha}\right)=0.63+1.03 \cdot \log \left(L[\mathrm{O} \text { II }]_{\text {,dered,42 }}\right)
\end{aligned}
$$

where $L[\mathrm{O} \text { II }]_{\text {,uncor,42, }} L[\mathrm{O} \text { II }]_{\text {,dered,42 }}$ are the measured and dereddened [O II] luminosity in units of $10^{42} \mathrm{erg} \mathrm{s}^{-1}$, respectively. The $1 \sigma$ dispersions in these relations are 0.44 dex for the dustuncorrected and 0.19 dex for the dust-corrected luminosity. Even though the scatter is much lower in the latter case, the former is useful in most practical cases. For a reliable dust-correction, the measurement of at least two Balmer lines is required, which in itself provides a much more reliable SFR than through Eq. (1).

Figure 7 also illustrates the limitation of this procedure. Since $[\mathrm{O}$ II $](\lambda 3727 \AA)$ is strongly attenuated by dust, there is considerable scatter in the relation using the dust-uncorrected luminosity. Even though the calibration sample contains galaxies over nearly full range of metallicity probed by the observations $(8.2<12+\log (\mathrm{O} / \mathrm{H})<8.9)$, the uncorrected Eq. (1) will significantly under-predict the SFR for metal-rich $(12+\log (\mathrm{O} / \mathrm{H})>$ 8.7) galaxies because of their high dust content. If a reliable dustcorrection is available, the dust-corrected Eq. (1) then provides SFRs with an average dispersion of 0.2 dex over the full metallicity range probed.

For those cases where no dust correction can be performed, i.e., only one Balmer line is detected in the spectrum, we derive for $\mathrm{H} \alpha$ (based on 61 individual galaxies with $8.2<12+$ $\log (\mathrm{O} / \mathrm{H})<8.9)$

$$
\log \left(S F R_{\mathrm{H} \alpha}\right)=0.92+1.04 \cdot \log \left(L\left(\mathrm{H} \alpha_{\text {,uncor }, 42}\right)\right)
$$

with a dispersion of 0.25 dex. For $\mathrm{H} \beta$ the equivalent relation is

$$
\log \left(\operatorname{SFR} R_{\mathrm{H} \alpha}\right)=1.47+0.98 \cdot \log \left(L\left(\mathrm{H} \beta_{\text {,uncor, } 42}\right)\right)
$$

with a scatter of 0.36 dex from 57 galaxies. For the Balmer lines, dust-corrected relations are by construction equivalent to the one of Kennicutt (1998).

For the $[\mathrm{O}$ III $](\lambda 5007)$ line, the scaling relations are

$$
\begin{aligned}
& \log \left(S F R_{\mathrm{H} \alpha}\right)=0.98+0.81 \cdot \log \left(L[\mathrm{O} \text { III }]_{\text {,uncor,42 }}\right) \\
& \log \left(S F R_{\mathrm{H} \alpha}\right)=0.71+0.92 \cdot \log \left(L[\mathrm{O} \mathrm{III}]_{\text {,dered,42 }}\right)
\end{aligned}
$$

with a dispersion of 0.42 dex for the uncorrected, and 0.28 dex in the dereddened case (from 57 individual galaxies in total). The relatively large dispersion, even after removing the effect of dust reddening, illustrates that $[\mathrm{O} \mathrm{III}]$ is the least reliable SF-tracer considered in this study. In the following, we use the emissionline that yields the smallest uncertainty in SFR when propagating $1 \sigma$ dispersions accordingly when necessary (typically first

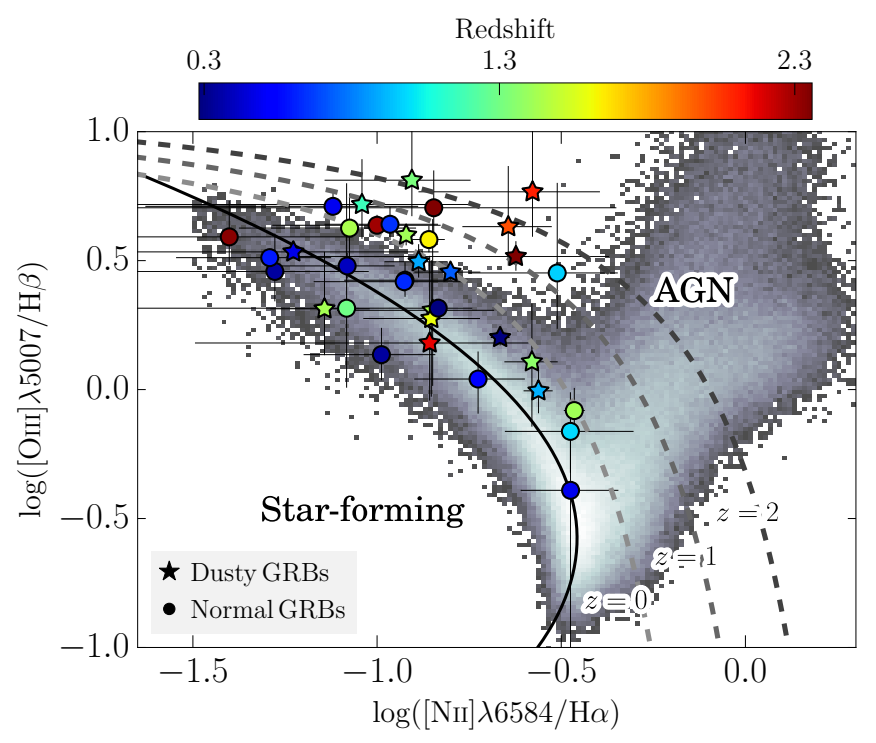

Fig. 8. Baldwin-Phillips-Terlevich (BPT) classification diagram for GRB hosts. The background sample is the logarithmic space density of local $(z \sim 0)$ SDSS galaxies with line-fluxes from the MPA/JHU cat$\operatorname{alog}^{11}$. Colored circles are 34 individual GRB-selected galaxies with redshifts indicated by the color bar. The increasingly darker dashed gray lines are the redshift-dependent differentiation lines between starforming galaxies and AGN of Kewley et al. (2013b) at the indicated redshifts. The black solid line is the ridge line, i.e., the line with the highest density of SDSS galaxies. Dusty/normal GRBs are indicated by stars/circles.

$\mathrm{H} \alpha$, then $\mathrm{H} \beta$, [O II] , and [O III] only if no other emission line is detected).

It is immediately clear that the scaling relations detailed above depend on the distribution of galaxy properties in the reference sample. Or, in other words, we consider these scaling relations most useful and accurate for low-mass, high-redshift, star-forming galaxies which have the average color excess of our sample $\left(A_{V} \sim 0.8 \mathrm{mag}\right.$ or $E_{B-V} \sim 0.25 \mathrm{mag}$, Sect. 5.3).

\section{Physical properties of the GRB host population}

\subsection{GRB hosts in the BPT diagram}

Figure 8 shows the GRB hosts in the Baldwin-Phillips-Terlevich (BPT) diagram (Baldwin et al. 1981) that allows emission-line objects to be separated into star-forming galaxies and AGNs based on the $[\mathrm{N} \mathrm{II}] / \mathrm{H} \alpha$ and $[\mathrm{O}$ III $] \lambda 5007 / \mathrm{H} \beta$ ratios (e.g., Kewley et al. 2001). In our sample, 34 GRB hosts have adequate constraints on the four emission-line fluxes to establish their position in the BPT diagram. As we are interested in line-flux ratios, we use here (and in the following whenever line-ratios are important) only the statistical errors from Tables 2 and 3 .

The redshift range of the GRB hosts extends from $z=0.3$ to $z=2.5$. As background sample, we plot the logarithmic space density of 330000 local $(z \sim 0)$ SDSS (Abazajian et al. 2009) galaxies from the MPA-JHU catalogue (e.g., Kauffmann et al. 2003), as well as the redshift-dependent classification lines between AGN and star-forming galaxies as gray dashed lines (Kewley et al. 2013b). The ridge line of SDSS galaxies (Brinchmann et al. 2008) is shown in black.

Our GRB hosts occupy a different phase-space than SDSS galaxies in the BPT diagram because they are mostly

11 http://www . mpa-garching .mpg.de/SDSS 


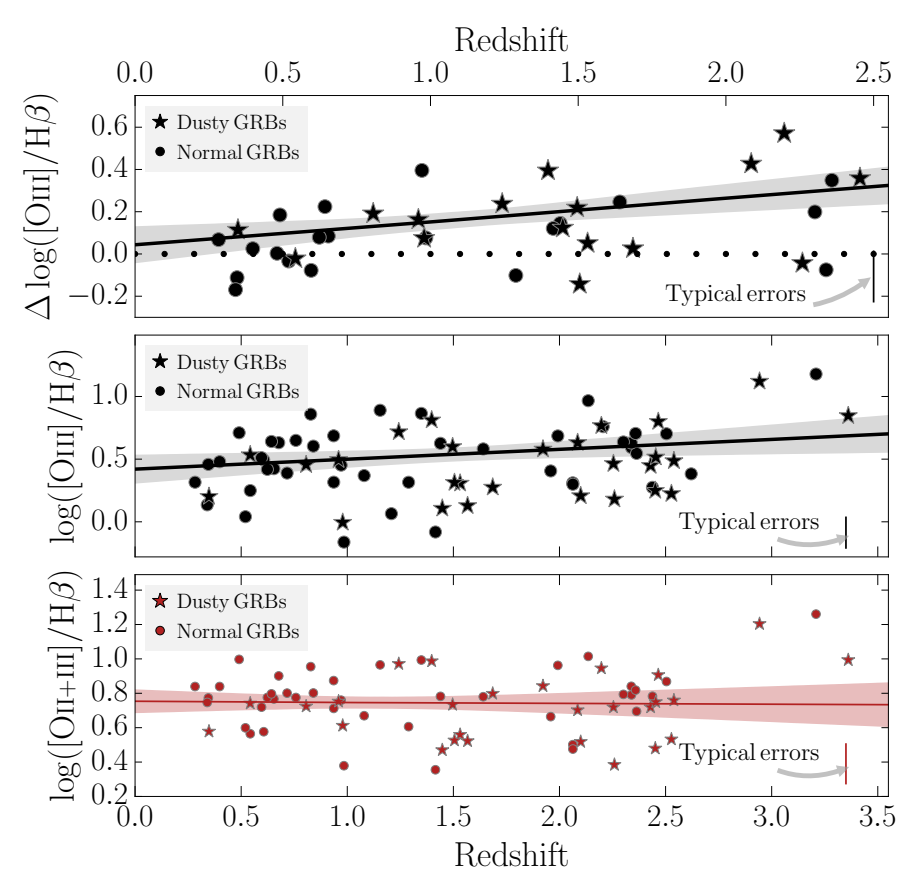

Fig. 9. Minimum difference of the GRB hosts from the ridge-line (illustrated by the dots) of $z \sim 0$ SDSS star-forming galaxies (top panel). We show $[\mathrm{O} \mathrm{III}] / \mathrm{H} \beta$ in black and $([\mathrm{O} \mathrm{III}]+[\mathrm{O} \mathrm{II}]) / \mathrm{H} \beta$ in red as a function of $z$ in the middle and bottom panel, respectively. In all panels, solid lines denote the most-likely linear regression, with regions of $68 \%$ probability in shaded areas. Dusty/normal GRBs are indicated by stars/circles. Error bars are omitted to enhance clarity and replaced by typical errors in the bottom-right corner.

above the ridge line which denotes the highest density of local star-forming galaxies. A similar offset is well-observed in many high redshift galaxy samples (e.g., Shapley et al. 2005; Kriek et al. 2007; Steidel et al. 2014) and also for galaxies hosting H-poor super-luminous supernovae (Leloudas et al. 2015). This offset is often attributed to harder ionization fields, higher ionization parameters or changes in the ISM properties (e.g., Brinchmann et al. 2008; Kewley et al. 2013a; Steidel et al. 2014).

There is some evidence of an evolution of the position of the targets in the BPT diagram with redshift. The top and middle panel of Fig. 9 show the minimum difference to the SDSSbased ridge line $(\Delta([\mathrm{O} \mathrm{III}] / \mathrm{H} \beta))$ as well as $[\mathrm{O} \mathrm{III}] / \mathrm{H} \beta$. On average $\Delta([\mathrm{O} \mathrm{III}] / \mathrm{H} \beta)$ and $[\mathrm{O} \mathrm{III}] / \mathrm{H} \beta$ increase by $0.11 \pm 0.02$ and $0.08 \pm 0.03$ dex per unit redshift. These values are derived as the median of all stochastic samples (Sect. 2.4) with errors representing the region of $68 \%$ likelihood of all trials. The fractions of samples producing no evolution in $[\mathrm{O} \mathrm{III}] / \mathrm{H} \beta$, and $\Delta([\mathrm{O} \mathrm{III}] / \mathrm{H} \beta)$ are $p=0.015$ and $p=0.0018$, respectively. A rising trend is not seen (bottom panel of Fig. 9) in the ratio of the summed oxygen line flux $([\mathrm{O} \mathrm{III}]+[\mathrm{O} \mathrm{II}])$ to $\mathrm{H} \beta(0.01 \pm 0.04$ dex per unit redshift).

Changes in the metallicity of the nebular gas are unlikely to play the dominant role in the displacement between highredshift and local galaxies as this would move galaxies primarily along the ridge line. Photoionization models from Steidel et al. (2014), for example, predict very little contribution from metallicity changes to the observed offset in the BPT diagram. In addition, the metallicity distributions of the sample are comparable at $z<1$ and $1<z<2$ (Sect. 5.6.2), indicating that the larger difference of galaxies at higher redshift is not driven by a decreasing gas-phase oxygen abundance.
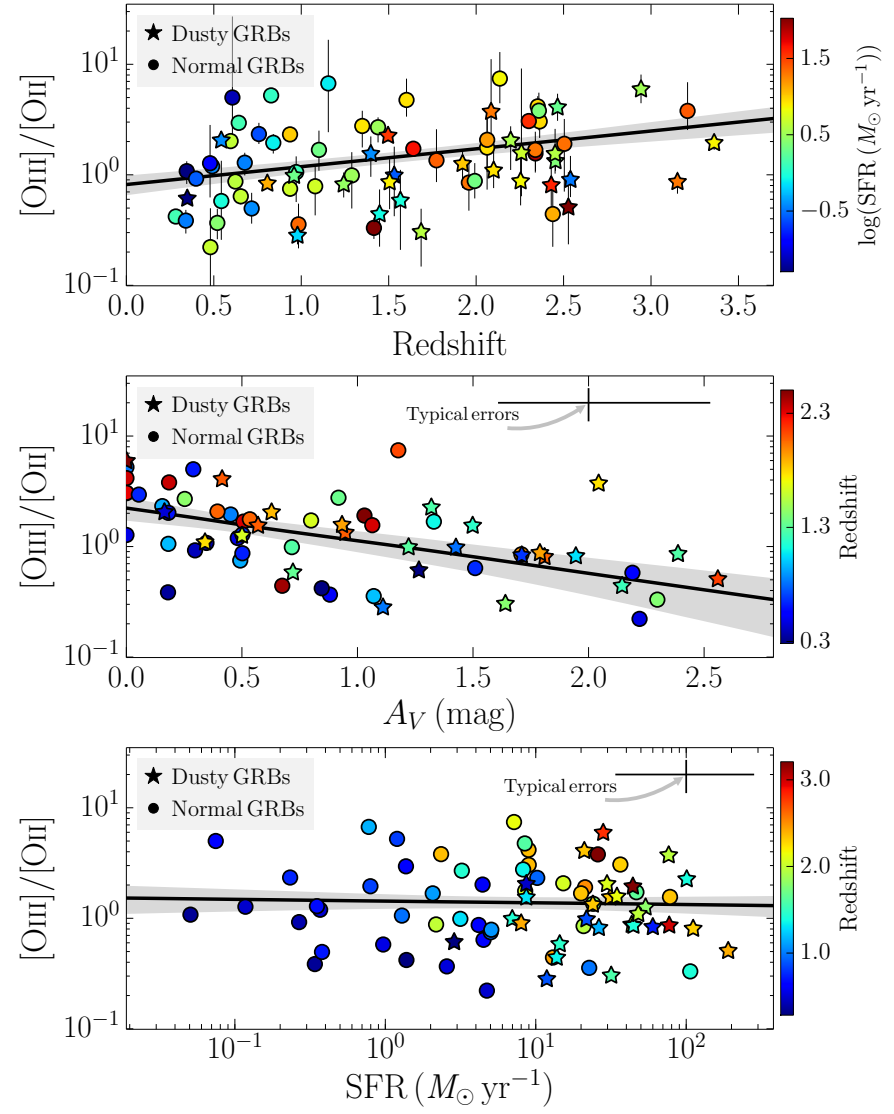

Fig. 10. $[\mathrm{O} \mathrm{III}] /[\mathrm{O} \mathrm{II}]$ as a function of (from top to bottom): redshift, galaxy $A_{V}$, and SFR. The color of individual data points are according to the color bars. In all panels, solid lines denote the most-likely linear regression, with regions of $68 \%$ probability in shaded areas. Dusty/normal GRBs are indicated by stars/circles.

Our observations are thus consistent with the hypothesis that the difference in the location in the BPT diagram between GRB hosts and $z \sim 0$ star-forming galaxies is caused by an increase in the ionization fraction, i.e., for a given metallicity a larger percentage of the total oxygen abundance is present in higher ionization states at higher redshifts. This could be caused by a harder ionization field originating for example from hot O-type stars (Steidel et al. 2014) that emit a large number of photons capable of ionizing oxygen into [O III].

\subsection{Ionization evolution}

The ratio of $[\mathrm{O} \mathrm{III}] /[\mathrm{O}$ II $]$, which we plot in Fig. 10 against redshift, $A_{V}$, and SFR, is often used as a proxy for the ionization parameter. A rising $[\mathrm{O} \mathrm{III}] /[\mathrm{O} \mathrm{II}]$ ratio with redshift is evident from 79 events with adequate wavelength coverage to constrain both [O III] and [O II]. [O III]/[O II] increases from 0.9 at $z \sim 0.3$ by a factor of 3 to $\sim 3$ at $z \sim 3.3$. The fraction of iterations returning no correlation between the $[\mathrm{O} \mathrm{III}] /[\mathrm{O} \mathrm{II}]$ ratio and redshift is $p=0.0018$ for the full sample and $p=0.0039$ when restricting the redshift range to $z<2.7$.

The predicted $[\mathrm{O} \mathrm{III}] /[\mathrm{O} \mathrm{II}]$ ratio at $z=3.3$ is remarkably similar to the ratio of the composite LBG spectrum at $z \sim 3.5$ (Maiolino et al. 2008; Troncoso et al. 2014). The evolution in [O III]/[O II] is again unlikely to be caused by metallicity effects (lower metallicity galaxies have higher [O III $] /[\mathrm{O}$ II $]$ ratios) 
because there is no statistically significant difference seen in metallicity between the $z<1$ and $1<z<2$ sample (Sect. 5.6).

Even though both the ionization parameter and SFR are correlated with redshift, the bottom panel in Fig. 10 shows no obvious relation between $[\mathrm{O} \mathrm{III}] /[\mathrm{O}$ II] and SFR (Sect. 5.4). It does show (middle panel in Fig. 10) an anti-correlation with dust content $\left(A_{V}=0.35-0.90 \cdot \log ([\mathrm{O} \mathrm{III}] /[\mathrm{O}\right.$ II $])$, with $\left.p=0.037\right)$. The dust content ${ }^{12}$ as parametrized through a color-excess $E_{B-V}$ or visual attenuation $A_{V}$ of a galaxy (Sect. 5.3) correlates with stellar mass $M_{\star}$, where $A_{V}=1 \mathrm{mag}$ corresponds roughly to $M_{\star} \sim 10^{10} M_{\odot}$ (e.g., Zahid et al. 2014).

The $[\mathrm{O}$ III $] /[\mathrm{O}$ II] ratio thus increases with redshift, whereas it decreases with $A_{V}$ (or stellar mass), plausibly an effect of the efficient absorption of ionizing photons on dust. The net effect makes $[\mathrm{O} \mathrm{III}] /[\mathrm{O} \mathrm{II}]$ independent of SFR in our sample.

\subsection{Dust properties - distribution and redshift evolution of galaxy $A_{V}$}

We measure the rest-frame attenuation of light from dust through comparing the observed Balmer decrement $r_{\text {obs }}$ with the theoretical expectation $r_{\text {theo }}$ for a given electron temperature. To exploit the broad wavelength coverage and detection of more than two Balmer lines, we simultaneously minimize the various expected ratios against $A_{V}$ for the four strongest Balmer lines. At an electron temperature of $10^{4} \mathrm{~K}$ and density of $10^{2} \mathrm{~cm}^{-3}$ to $10^{4} \mathrm{~cm}^{-3}$ these ratios are (Osterbrock 1989) $\mathrm{H} \alpha / \mathrm{H} \beta=2.87$, $\mathrm{H} \alpha / \mathrm{H} \gamma=6.17$, and $\mathrm{H} \alpha / \mathrm{H} \delta=11.24$. In a Milky-Way-type extinction law ${ }^{13}$ (Pei 1992) with $R_{V}=3.08$, the wavelengths of the four lines correspond to $A_{\mathrm{H} \alpha}=0.79 \mathrm{mag}, A_{\mathrm{H} \beta}=1.16 \mathrm{mag}$, $A_{\mathrm{H} \gamma}=1.31 \mathrm{mag}, A_{\mathrm{H} \delta}=1.381 \mathrm{mag}$ for $A_{V}=1 \mathrm{mag}$. For the Balmer decrement of $\mathrm{H} \alpha$ over $\mathrm{H} \beta$ follows

$A_{V}=\frac{-2.5 \cdot \log \left(r_{\mathrm{obs}} / r_{\text {theo }}\right)}{A_{\mathrm{H} \alpha}-A_{\mathrm{H} \beta}}$,

and similarly for the other ratios. In the calculation of $A_{V}$, we use a prior of $A_{V} \geq 0 \mathrm{mag}$, and provide the median of the a-posteriori probability and $1 \sigma$ equivalent parameter range as error to represent its distribution. In total, we constrain $A_{V}$ for 71 events in the sample (Table 4), the distribution of which is shown in Fig. 11.

After applying the statistical analysis and cuts of Sects. 2.3.3 and 2.4, the median $A_{V}$ of our sample is $A_{V}^{\text {med }}=0.8_{-0.3}^{+0.4} \mathrm{mag}$ and seems to be somewhat evolving with redshift (Fig. 11). At redshifts lower than $z<1$, there is a lower number of $A_{V}>1.5 \mathrm{mag}$ hosts when compared to the $z>1$ sample, and we measure $A_{V}^{\text {med }}=0.55_{-0.18}^{+0.24} \mathrm{mag}$. This value is fully consistent with the value reported in Savaglio et al. (2009). $A_{V}^{\text {med }}$ increases to $A_{V}^{\text {med }}=1.2 \pm 0.3 \mathrm{mag}$ at $1<z<2$. It is $A_{V}^{\text {med }}=0.7_{-0.2}^{+0.3}$ mag at $z>2$, but we note that the galaxies are significantly brighter than comparison samples of GRB hosts in this redshift range.

The significance of this evolution in $A_{V}$ with redshift however is relatively modest: comparing the two distributions centered around $z \sim 0.6(z<1)$ and $z \sim 1.5(1<z<2)$ with each other (Fig. 11), the null-hypothesis that the two distributions are drawn from the same parent sample can be rejected with a significance of $2 \sigma$. At even higher redshifts further selection effects

\footnotetext{
${ }^{12}$ Here, and in the following, $E_{B-V}$ or $A_{V}$ always refers to the rest-frame color excess/visual attenuation at the redshift of the target galaxy.

${ }^{13}$ We choose a MW-type extinction law to compare directly with other studies, and note that the difference to extinction laws from the SMC or LMC is small in the wavelength range of $\mathrm{H} \delta$ to $\mathrm{H} \alpha$.
}

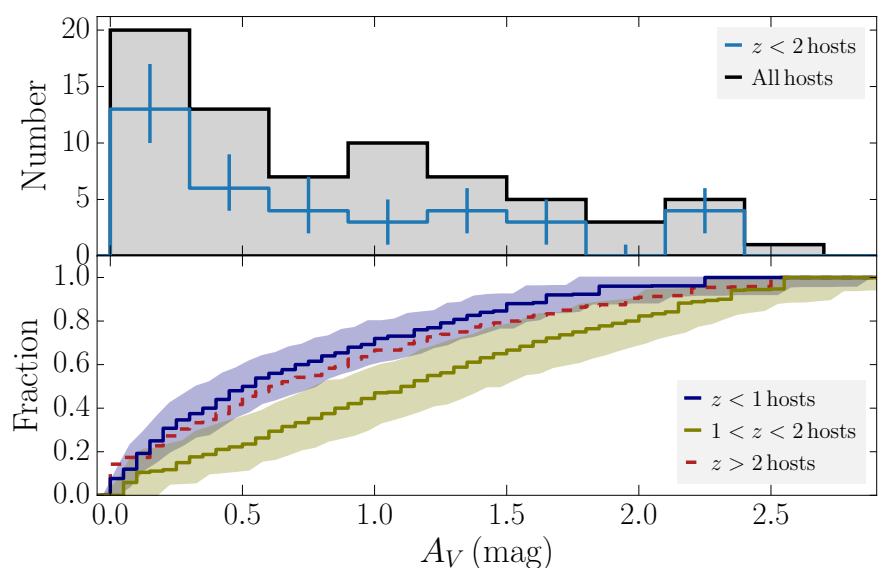

Fig. 11. Blue solid histogram and error-bars in the top panel $A_{V}$ distribution for $z<2$ GRB from the statistical analysis (Sect. 2.3.3 and Sect. 2.4). The black histogram represents all raw measurements without corrections. Lower panel: the cumulative distribution of $A_{V}$ and its uncertainty from $z<1$ hosts (dark blue) and $1<z<2$ hosts (olive). The red dashed line shows $z>2$ hosts (error region not shown).

play an important role as well: high $A_{V}$ hosts are not appearing because of the decreasing sensitivity SFR at high $A_{V}$ (Fig. 5).

The relative sparsity of high $A_{V}$ events at low redshift is however not easily explained by selection effects. At the average mass of the SF-weighted galaxy population of $M_{\star} \sim 10^{10} M_{\odot}$ at $z \sim 0.6$ (Perley et al. 2013b; Hunt et al. 2014), the average reddening for galaxies is $E_{B-V} \sim 0.35 \mathrm{mag}$ (Zahid et al. 2014, and references therein). For GRBs to trace star formation representatively, half of the sample would be expected above this value and half below, while we observe $(82 \pm 8) \%$ of all hosts below $E_{B-V}=0.35$ mag at $z<1$.

Although there are indications of an increasing dust-content in GRB hosts with redshift, the low significance prevents us from reaching stronger conclusions. Interestingly, however, a very similar trend - highest visual extinctions at $z \sim 1.5-2-$ exists in the complete sample of $A_{V}$ values derived from afterglow data (Covino et al. 2013) and in the evolution of the far UV attenuation in field galaxies (Cucciati et al. 2012). It is also consistent with the somewhat higher fraction of dusty GRBs at higher redshift (Sect. 2.3.3).

\subsection{Star formation rates}

As introduced in Sect. 4.4, we can use the flux of nebular lines to derive constraints on the current star formation rate (SFR). $\mathrm{H} \alpha$, in particular, is a common tracer of SF on short timescales of a few million years, and its dependence on the uncertainty in the dust-obscuration is only modest.

All SFRs are given in Table 4 and are corrected for the measured $A_{V}$ if available (Sect. 5.3), or derived from scaling relations that intrinsically include the effect of dust (Sect. 4.4). Here we also add the systematic uncertainty of the flux calibration (Tables 2 and 3). In the majority of all cases, however, the error budget is dominated by the uncertainty in the dust correction.

Generally, all of the hosts in the sample are forming stars at a level of at least $0.02 M_{\odot} \mathrm{yr}^{-1}$. Even though we are sensitive to galaxies with a very low SFR at $z<0.5$, we detect none (but see Rossi et al. 2014, for a quiescent galaxy at the position of GRB 050219A). Figure 12 shows the distribution of SFRs for 89 hosts, out of which 68 are based on $\mathrm{H} \alpha$, ten on $\mathrm{H} \beta$, eight on [O II], and three on [O III]. The distribution is very broad, 


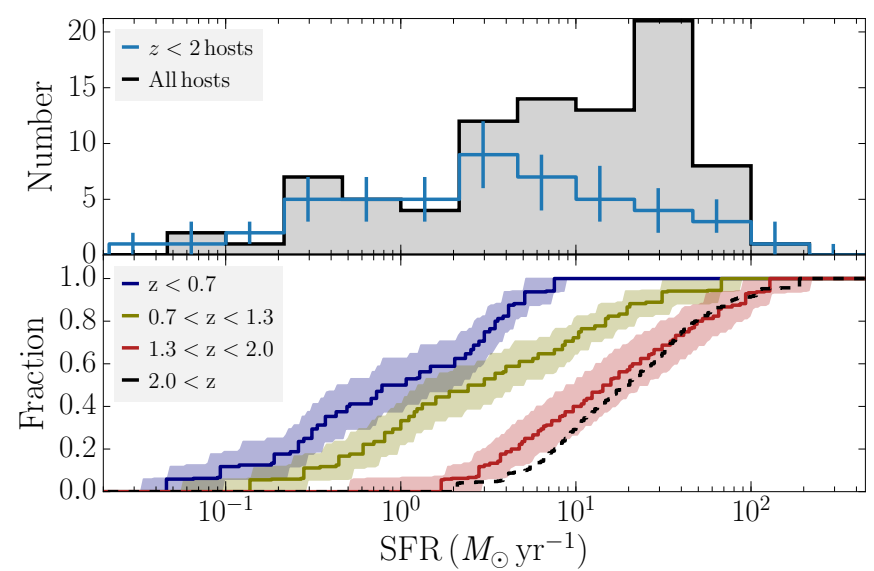

Fig. 12. Blue solid histogram with error bars in the top panel: SFR distribution and its uncertainty of $z<2$ GRBs, where we have applied the correction for the over-proportionality of dusty GRBs in the sample (Sects. 2.3.3 and 2.4). Black data show all raw measurements without corrections. Lower panel: cumulative distributions of SFR and its uncertainty of $z<0.7$ hosts (dark blue), $0.7<z<1.3$ hosts (olive) and, $1.3<z<2.0$ hosts (red), in the same way corrected as the blue histogram. The black dashed line denotes hosts at $z>2$.

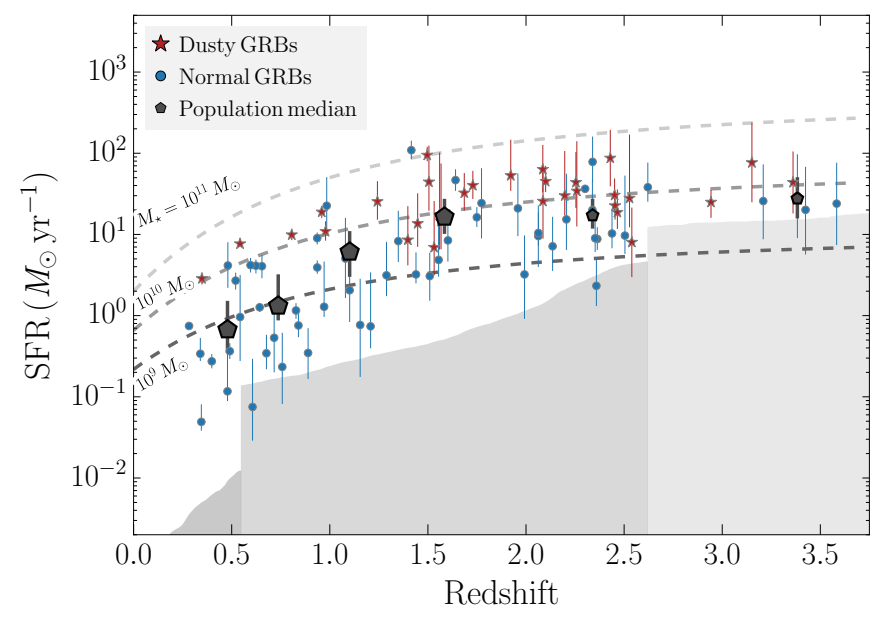

Fig. 13. SFR as a function of redshift. Blue circles and red stars represent individual galaxies. Large or small pentagons are the median values binned in redshift with bootstrapped errors at $z<2$ and $z>2$, respectively. Here, we have applied the correction for the over-proportionality of dusty GRBs in the sample (Sects. 2.3.3 and 2.4). The gray shaded areas represent the average sensitivity as detailed in Sect. 3.4 using $A_{V}=1 \mathrm{mag}$ (Sect. 5.3). The three dashed lines show typical SFRs for differently massive galaxies from the main sequence (Speagle et al. 2014) for a qualitative estimate of the evolution of field galaxies.

spanning nearly four orders of magnitude between $0.05 M_{\odot} \mathrm{yr}^{-1}$ up to around $200 M_{\odot} \mathrm{yr}^{-1}$ and peaks at $20 M_{\odot} \mathrm{yr}^{-1}$. A large number of galaxies with high SFRs either host dusty GRBs or are at $z>2$ (where selection effects are important). The peak of the corrected SFR distribution for $z<2$ GRBs (blue lines in Fig. 12) is therefore an order of magnitude lower $\left(2 M_{\odot} \mathrm{yr}^{-1}\right)$.

\subsubsection{Redshift evolution of star formation rates}

As shown in Figs. 12 and 13, there is clear evolution of the typical SFR of GRB hosts with redshift. While at $z \sim 0.5$, the median SFR of a GRB host is $\sim 0.6 M_{\odot} \mathrm{yr}^{-1}$ (see also Savaglio et al. 2009 ), it rapidly increases by a factor of 25 to $\sim 15 M_{\odot} \mathrm{yr}^{-1}$ at $z \sim 2$. Above $z>2$, no further strong rise is evident in the data, and the median SFR levels out at around $\sim 20 M_{\odot} \mathrm{yr}^{-1}$.

This behavior changes only slightly if we take into account that our selection might miss the faintest $\sim 20 \%$ of hosts (total of $\sim 5$ ) at $1<z<2$ (Sect. 2.3 and Fig. 2), or that we have initially excluded four spectra of galaxies that could potentially be in this redshift range (Sect. 2.1).

The $30-40 \%$ quantile of SFRs at $z>1.3$ is still an order of magnitude larger than the median SFR at low redshifts (Fig. 12, lower panel). To be consistent at the $90 \%$ confidence level with no evolution in SFR, we would need to have missed by chance more than 40 GRBs (i.e., twice the sample size in the $1.3<z<$ 2.0 range) that are hosted by galaxies with $S F R \lesssim 2 M_{\odot} \mathrm{yr}^{-1}$.

At least at $z>2.5$ there is an obvious effect of sensitivity (shaded area in Fig. 13): only the most star-forming hosts $\left(>20 M_{\odot} \mathrm{yr}^{-1}\right.$ ) have emission lines bright enough to be picked up by X-Shooter. We hence sample only the brightest end of the SFR distribution, and the comparison to the $z<2$ data becomes less meaningful. It is however clear that there is no further order-of-magnitude increase in the median SFR of GRBselected galaxies above $z>2$.

The strong evolution in the SFR is not particularly surprising: indeed it is similar to the redshift dependence of the UV luminosity of normal galaxies parametrized with e.g., the normalization of the main sequence (e.g., Wuyts et al. 2011; Whitaker et al. 2012). To illustrate the evolution of field galaxies, we also plot redshift tracks for galaxies on the main sequence with stellar masses between $M_{\star}=10^{9} M_{\odot}$ and $10^{11} M_{\odot}$ in dashed lines in Fig. 13. They indicate qualitative (but not quantitative) agreement in the relative rise in SFR with redshift between the different samples. There are significant differences, in particular because of a relative lack of galaxies in the high SFR regime at low redshift.

\subsubsection{Sparsity of high SFR GRB hosts at $z<1$}

We illustrate the sparsity of high SFR galaxies hosting GRBs at low redshifts in Fig. 14 using the GRB host sample between $z=$ 0.55 and $z=1.05(\langle z\rangle=0.81)$ and the dust-corrected luminosity function (LF) of $\mathrm{H} \alpha$ from Ly et al. (2011) at a similar redshift (Schechter parameters $\alpha=1.6$ and $\left.\log \left(L_{*} /\left(\mathrm{erg} \mathrm{s}^{-1}\right)\right)=43.0\right)$. We multiply the LF by the SFR (or, in this case, $\mathrm{H} \alpha$ luminosity) since GRBs are expected to follow total SFR and not galaxy numbers.

The SFR-weighted $\mathrm{H} \alpha-\mathrm{LF}$ then represents the distribution that would be obtained from GRB hosts if they directly traced all star formation. Figure 14 shows, however, a strong discrepancy between both distributions (see also Boissier et al. 2013) with the GRB hosts peaking at around an order of magnitude lower SFRs $\left(\sim 1 M_{\odot} \mathrm{yr}^{-1}\right)$ than the SFR-weighted $\mathrm{H} \alpha-\mathrm{LF}\left(\sim 10 M_{\odot} \mathrm{yr}^{-1}\right)$. There is no observational bias in the sample that could explain this discrepancy.

\subsection{Line broadening}

The broadening of the nebular lines traces the turbulent and rotational motion of the hot gas within the gravitational potential of the host galaxy. The line-widths are well resolved in all but a few low-redshift cases because of the medium resolving power (4000 $\lesssim R 10000$ ) of X-Shooter. The line-broadening from long-slit spectroscopy correlates reasonably well with velocity dispersions measured in spatially resolved data (Richard et al. 2011), so we directly use the line-width $\sigma$ as a proxy 


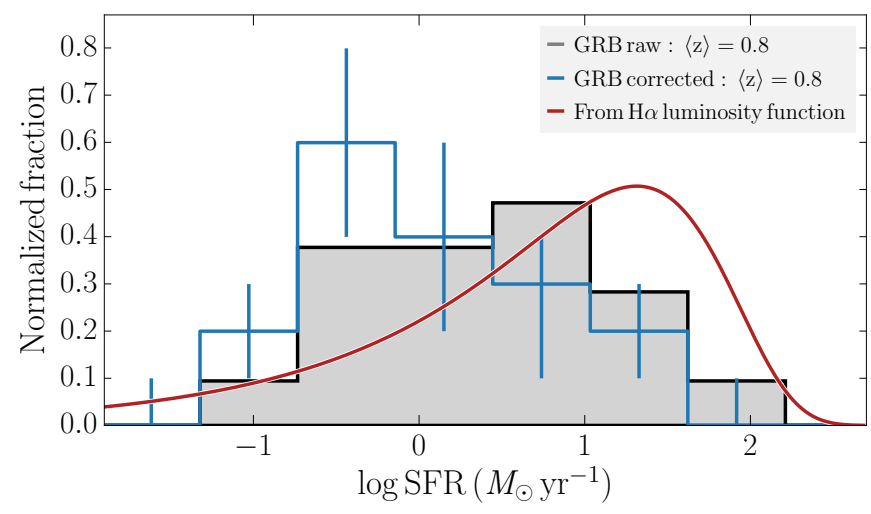

Fig. 14. SFR-weighted $\mathrm{H} \alpha$-luminosity function at $z=0.8$ (Ly et al. 2011) in red compared to the histogram of SFRs from GRB hosts. The blue-solid histogram with error bars shows the SFR distribution and its uncertainty of our $0.55<z<1.05$ GRBs, where we have applied the correction for the over-proportionality of dusty GRBs in the sample (Sects. 2.3.3 and 2.4). Black data are raw measurements in the respective redshift range without corrections. Shown curves are normalized to an area of 1 .

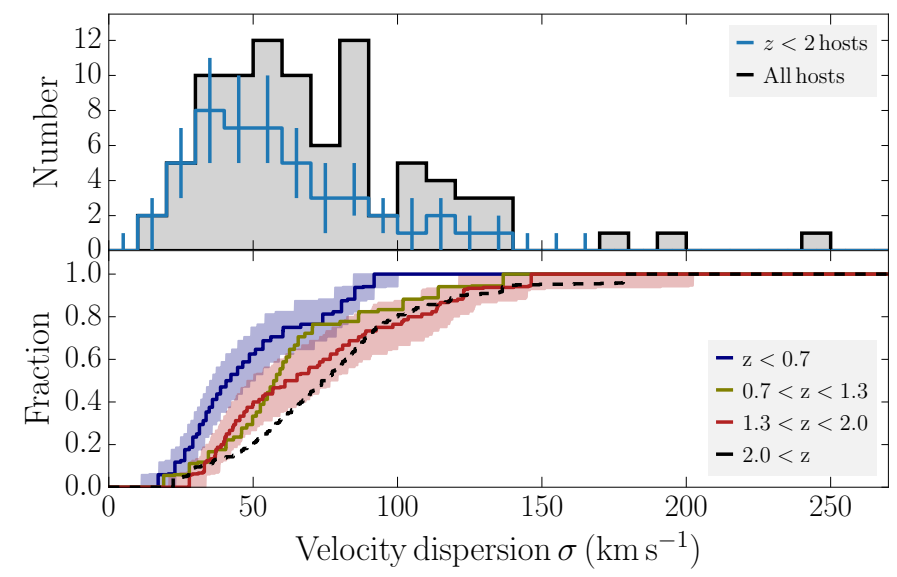

Fig. 15. Blue histogram with error bars in the top panel: distribution of line broadenings $(\sigma)$ and its uncertainty of $z<2$ GRBs (Sects. 2.3.3 and 2.4). The black histogram shows raw measurements without corrections. Bottom panel: plot cumulative distributions of $\sigma$, where black/blue/red colors denote redshift intervals centered around of $z \sim 0.5, z \sim 1.1$, and $z \sim 1.6$, respectively. To enhance clarity, the error regions around the $z \sim 1.1$ and $z>2$ histogram are not shown.

for the gas velocity dispersion $\sigma_{\mathrm{d}}$. For the bulk of our targets, no evidence for rotation is seen in the spectra, but we caution that the rotational component contributes to the measured line-broadening, in particular at higher stellar masses (e.g., Förster Schreiber et al. 2009).

The virial or dynamical mass $M_{\mathrm{d}}$ of a galaxy is then a function of galaxy geometry, velocity dispersion $\sigma_{\mathrm{d}}$, and typical physical extent, or half-light radius $r_{\mathrm{e}}$ of the galaxy via the virial theorem (see, e.g., Pettini et al. 2001) as $M_{\mathrm{d}}=C \sigma_{\mathrm{d}}^{2}\left(r_{\mathrm{e}} / G\right)$. $C$ is related to the galaxy's geometry, density, and velocity anisotropy, with $C=5$ in the case of an isotropic sphere (Pettini et al. 2001), or $C \sim 3.4$ for a disk-like geometry (Erb et al. 2006b).

No measurements of the effective radius for the bulk of the sample is available to us because they are spatially unresolved from the ground. In addition, a systematic uncertainty is present at high redshift because the emission line shape is dominated by luminous H II regions, probing only a part of the gravitational
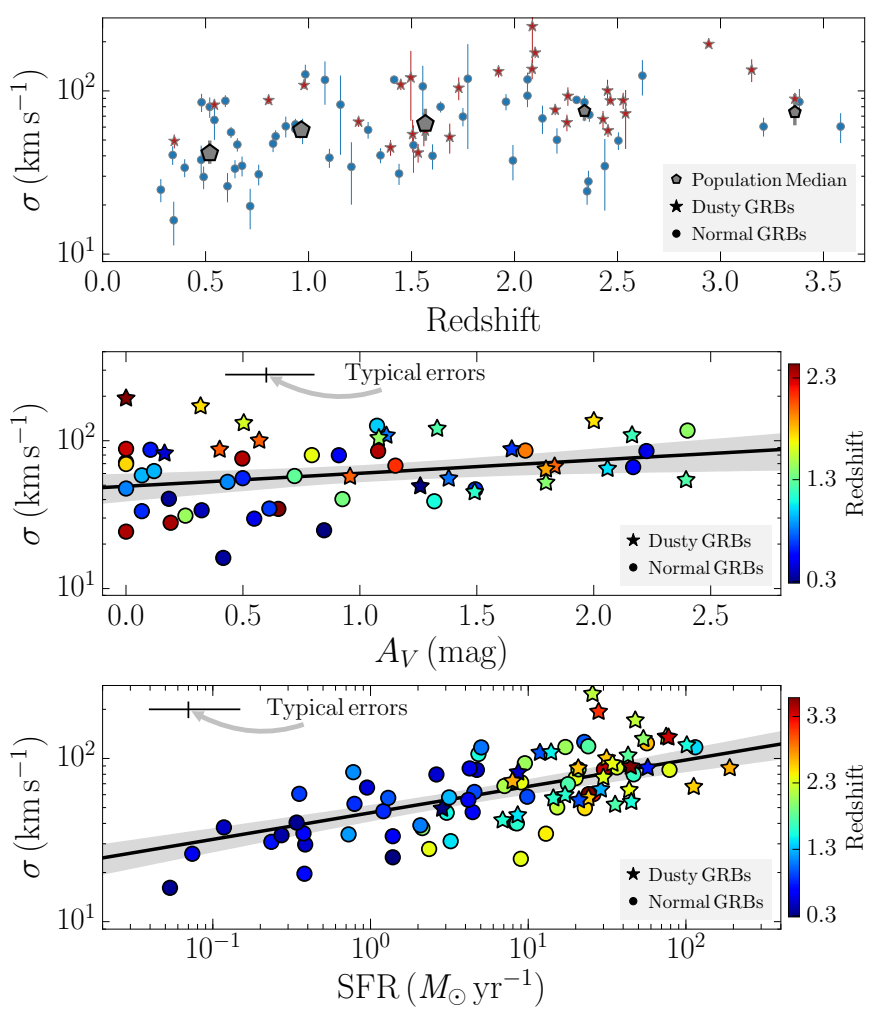

Fig. 16. Line broadening $\sigma$ as a function of (from top to bottom): redshift, $A_{V}$, and $\sigma$. In the top panel, large/small pentagons show the median values binned in redshift with bootstrapped errors at $z<2$ and $z>2$, respectively. The color coding of events corresponds to redshift, with the scale given by the color bar. In the lowest two panels, solid lines denote the most-likely linear regression, with error-regions $(68 \%$ probability) in shaded areas.

potential. Despite all these uncertainties, the simple velocity broadening is an important tracer of galaxy properties as demonstrated in Figs. 16 and 20.

The peak of the line-width distribution (Fig. 15) at $40 \mathrm{~km} \mathrm{~s}^{-1}$ suggests a dynamical mass on the order of $M_{\mathrm{d}} \sim 10^{9.4} M_{\odot}$, the lowest measured values $\left(20 \mathrm{~km} \mathrm{~s}^{-1}\right)$ imply $M_{\mathrm{d}} \sim 10^{8.7} M_{\odot}$. At the high $\sigma$ end, there are only three hosts above $\sigma=150 \mathrm{~km} \mathrm{~s}^{-1}$, namely GRB $070521(z \sim 2$ and with a large error on $\sigma)$, GRB $090201(z \sim 2)$, and GRB 120118B $(z \sim 3)$ which indicate high dynamical masses $\left(M_{\mathrm{d}} \sim 10^{11} M_{\odot}\right)$. These three galaxies are selected through dust-extinguished GRBs and their $\sigma$ is comparable to those of the most luminous galaxies at the respective redshift interval (e.g., Erb et al. 2006b).

With respect to redshift (Fig. 16), there are indications for an evolution such that lower redshift GRB hosts have - on average - a smaller line-broadening. In particular, there is a lack of high $\sigma$ galaxies at $z \lesssim 1$ (Fig. 15, lower panel), while there is no obvious bias that would prevent us from selecting or observing these galaxies in the first place. The average $\sigma$ increases qualitatively similar to the evolution of $A_{V}$ in Sect. 5.3 - from $\sigma_{\text {med }}=40 \mathrm{~km} \mathrm{~s}^{-1}$ at $z=0.5$ to $60 \mathrm{~km} \mathrm{~s}^{-1} \lesssim \sigma \lesssim 70 \mathrm{~km} \mathrm{~s}^{-1}$ at $z>1.0$. A mild correlation $(p=0.02)$ exists also between $A_{V}$ and $\sigma$, two quantities which relate to the total mass of a galaxy.

More obvious in Fig. 16 is the strong correlation between SFR and $\sigma\left(<10^{-6}\right.$ of all samples have no correlation $)$. A similar relation between luminosity and velocity dispersion $\left(L \propto \sigma_{\mathrm{d}}^{4}\right)$ is well-established in the local Universe. It was first observed in ellipticals by Faber \& Jackson (1976), and later also in $z \sim 2$ star-forming galaxies (Erb et al. 2006b). Murray et al. (2005) 
describe this correlation as a consequence of moderation of star formation activity through momentum-driven winds from supernovae together with radiation pressure. Star formation would drive-out gas above a critical luminosity $L_{\mathrm{M}} \propto 4 f_{\mathrm{g}} c / G \cdot \sigma_{\mathrm{d}}^{4}$, where $f_{\mathrm{g}}$ is the gas fraction. At the bolometric luminosity $L_{\mathrm{M}}$ a star-forming galaxy then self-regulates its SFR and reaches its maximum luminosity.

The correlation between SFR and $\sigma$ in Fig. 16 is consistent with $S F R \propto \sigma^{4}$, but there is considerable dispersion in the data. To test for a possible redshift evolution, we fit galaxies below and above $z=1$ with a fixed proportionality of $\sigma^{4}$. This returns within errors similar normalizations with minor evidence $(1.5 \sigma)$ for the higher-redshift galaxies shifted towards higher SFRs for a given $\sigma$. This would indicate higher gas-fractions in the model of Murray et al. (2005).

\subsection{Metallicity}

\subsubsection{Metallicity measurements from strong-line diagnostics}

One of the most fundamental - but also most difficult to measure - characteristic of galaxies is their metal abundance. While for GRB afterglows, metallicity can be probed directly and accurately in absorption at $z>1.8$ through their DLAs (e.g., Vreeswijk et al. 2004; Savaglio 2006; Prochaska et al. 2007; Ledoux et al. 2009), for faint (GRB-selected) galaxies it is unavoidable to resort to diagnostic ratios of strong emission lines (e.g., Erb et al. 2006a; Maiolino et al. 2008). These strong line diagnostics use metallicity-dependent ratios of e.g., [O II] and [O III] to H $\beta$ (e.g., Pagel et al. 1979; McGaugh 1991; Zaritsky et al. 1994), [N II] to $\mathrm{H} \alpha$ (Alloin et al. 1979; Pettini \& Pagel 2004), or [N II] to [O II] (e.g., Kewley \& Dopita 2002) to derive an oxygen abundance $12+\log (\mathrm{O} / \mathrm{H})$. For an extensive discussion on the strong line ratios, we refer to Kewley \& Dopita (2002).

In particular at high redshift, a reliable determination of the metal content in the hot gas phase becomes challenging through these line ratios (e.g., Maiolino et al. 2008). As we have seen in the previous paragraphs, the physical conditions, e.g., ionization parameter, SFR, possibly dust content, and gas-fractions are different from those of low-redshift galaxies on which most of the various strong-line diagnostics are calibrated. Currently, only very few directly measured abundances from high-redshift galaxies are available (e.g., Christensen et al. 2012) and none of the strong-line ratios are thus validated at $z \sim 2$. Any metallicity that is derived from these strong-line ratios must thus be taken cautiously (e.g., Steidel et al. 2014).

In addition, we are faced here with the problem that our sample spans a broad range of redshifts, broad range of SFRs and host masses, and a different set of lines is detected for every host. Because of the different calibrations inherent in different strong-line diagnostics, any comparison of them needs thorough consideration (e.g., Kewley \& Ellison 2008).

Given the aforementioned constraints, we decide to base our metallicity measurements on the single set of calibrators from Nagao et al. (2006) and Maiolino et al. (2008), which provide a homogeneous set of line diagnostics for different line ratios. Specifically, we use the five diagnostics based on $R_{23},[\mathrm{O}$ II] and [Ne III], [O III] and [N II], $\mathrm{H} \alpha$ and [N II], [N II] and [O II] and simultaneously minimize $12+\log (\mathrm{O} / \mathrm{H})$ against the available data. Via the line-flux measurements and errors (i.e., likelihood distributions of fluxes), we construct probability density functions (PDFs) for each diagnostic ratio, as well as one PDF for

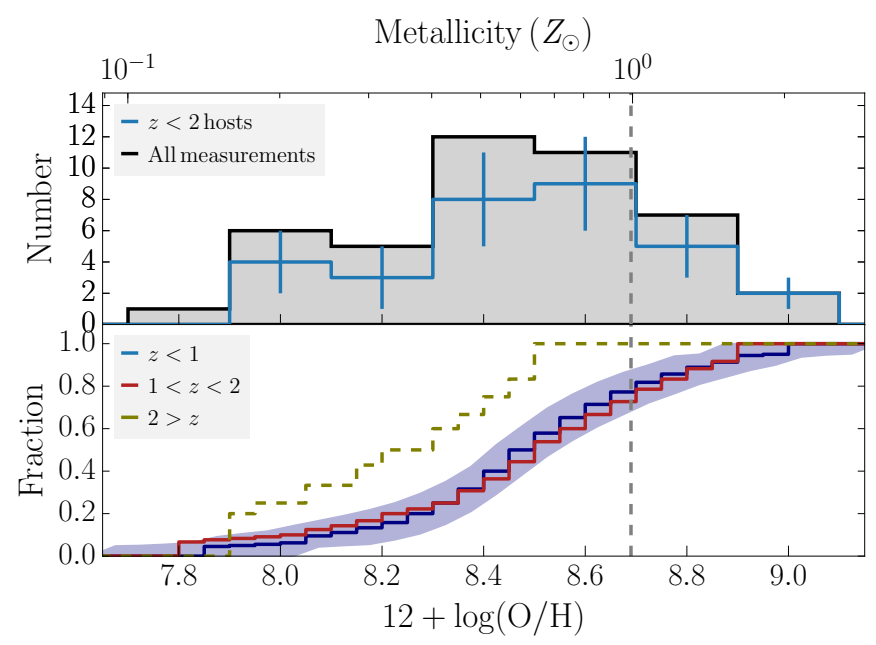

Fig. 17. Top panel: histogram of measured oxygen abundances $(12+$ $\log (\mathrm{O} / \mathrm{H}))$ derived through strong-line diagnostic ratios in blue following Sects. 2.3.3 and 2.4 and in black for all data. Bottom panel: cumulative distributions of the GRB host metallicities in different redshift intervals, where blue/red/olive colors denote redshift intervals below $z=1$ $\left(z_{\text {med }}=0.6\right), 1<z<2\left(z_{\text {med }}=1.4\right)$, and $2<z\left(z_{\text {med }}=2.4\right)$, respectively. $Z_{\odot}$ is indicated by the vertical, gray dashed line. Error-regions of the $1<z<2$ sample have a similar size to the one for $z<1$, but are not shown to enhance clarity.

the combination of them. Section B and Fig. B.1 provide more details on the procedure and individual events.

We also provide all line-flux measurements and errors in Tables 2 and 3, so oxygen abundances can be reproduced and recalculated on any given metallicity scale with the preferred strong-line diagnostic.

\subsubsection{Sample properties}

In total, we measure well-constrained gas-phase metallicities for 44 events (Table 4) in a redshift range between $z=0.3$ and $z=3.4$ with a median of $z_{\text {med }}=1.0$. The oxygen abundances are distributed between $12+\log (\mathrm{O} / \mathrm{H})=7.9(\mathrm{GRB} 120118 \mathrm{~B})$ and $12+\log (\mathrm{O} / \mathrm{H})=9.0(\mathrm{GRB} 051117 \mathrm{~B})$ with a peak of the distribution at $12+\log (\mathrm{O} / \mathrm{H}) \sim 8.5$ (Fig. 17).

Intriguingly, we detect a non-negligible fraction of GRB hosts at high metallicities $\left(Z \gtrsim Z_{\odot}\right.$, Fig. 17 , see also Levesque et al. 2010c; Elliott et al. 2013). It is thus obvious that there is no strict cut-off in host metallicities at least up to the solar value. We thus directly confirm the predictions from previous studies which indicated that GRBs form in various types of star-forming galaxies, even metal rich ones (Berger et al. 2007; Krühler et al. 2011; Perley et al. 2013b, 2015c).

The number of hosts with a metallicity above solar, however, is relatively small. From the sub-sample of galaxies with metallicity measurements, we derive fractions of $24 \pm 10 \%, 26 \pm 13 \%$, $7_{-4}^{+10} \%$ for $z<1,1<z<2$, and $2<z$. Under the assumption that the emission-line width $\sigma$ traces oxygen abundance as shown in Sect. 5.6.5, we can estimate metallicities also for those galaxies for which the strong-line diagnostics can not be applied. For instance, the host of GRB 050525A at $z=0.6$ is faint $(R=25.7 \mathrm{mag}$, Hjorth et al. 2012), has a narrow line width and low SFR, and is thus likely metal-poor (the correlations from Sect. 5.6.5 indicate $12+\log (\mathrm{O} / \mathrm{H}) \sim 8.2)$. When including all galaxies of this work, the fractions of super-solar metallicity hosts become $16 \pm 7 \%, 25 \pm 9 \%, 4_{-2}^{+5} \%$ at $z<1$, $1<z<2$, and $2<z$, respectively. 
At the highest redshifts $z \gtrsim 3$, emission-line metallicities are traditionally very expensive and challenging to obtain. We derive through observations of [Ne III] for GRBs 110818A $(z=3.36)$, $111123 \mathrm{~A}(z=3.15)$, and 120118B $(z=2.94)$ metallicities of $12+\log (\mathrm{O} / \mathrm{H})=8.25_{-0.25}^{+0.17}, 12+\log (\mathrm{O} / \mathrm{H})=8.01 \pm 0.28$, and $12+\log (\mathrm{O} / \mathrm{H})=7.89_{-0.17}^{+0.23}$, respectively. Comparing this with results on galaxy metallicities at $z \sim 3.5$ and above (Maiolino et al. 2008; Laskar et al. 2011), we see that those three events are probing the highest measured metallicities of LBGs at the given redshift. This is consistent with the significant line broadening and high SFRs (Sects. 5.5 and 5.4).

\subsubsection{Evolution with redshift}

To test for an evolution of the metal content in the gas-phase with redshift, we divide the galaxies into low $\left(z<1, z_{\text {med }}=0.6\right)$, medium $\left(1<z<2, z_{\text {med }}=1.4\right)$, and high redshift $(z>2$, $\left.z_{\text {med }}=2.4\right)$ samples and plot histograms of $12+\log (\mathrm{O} / \mathrm{H})$ in the bottom panel of Fig. 17. Remarkably, the cumulative distributions appear similar in the two low-redshift intervals. In contrast, the $z>2$ sample is somewhat shifted towards lower metallicities.

Cosmic-chemical evolution arguably proceeds most rapidly at higher redshifts. Between $z \sim 0$ and $z \sim 0.7$ the decrease in $12+\log (\mathrm{O} / \mathrm{H})$ for a galaxy of a given mass seems relatively modest at around 0.1-0.2 dex (Tremonti et al. 2004; Savaglio et al. 2005), even though this difference in redshift corresponds to over $5 \mathrm{Gyr}$ in the evolution of the Universe. The displacement of the mass-metallicity relation at high redshift is likely much larger than this, reaching $\sim 0.5 \mathrm{dex}$ at the high mass $\left(10^{11} M_{\odot}\right)$ end, and $1 \mathrm{dex}$ at $10^{9} M_{\odot}$ at $z \sim 3.5$ (Maiolino et al. 2008; Troncoso et al. 2014). This leads to relatively low gas-phase metallicities in the sample although we are only sensitive to the more luminous GRB hosts at $z>2$.

\subsubsection{Metallicity dependence of GRB hosts}

To understand the effects of metallicity on GRB progenitors and host galaxies and the implications for their ability to trace star formation, we summarize the primary results from the previous sections: first, GRB hosts with oxygen abundances of $12+\log (\mathrm{O} / \mathrm{H}) \sim 8.5$ are common in our sample. Second, the $z<1$ and $1<z<2$ GRB host metallicity distributions appear to be very similar. Third, there is a modest fraction $(\sim 20 \%$ at $z<1)$ of GRBs in $Z>Z_{\odot}$ galaxies. And last, the maximum metallicity of the most luminous GRB hosts at $z \sim 3$ is $12+\log (\mathrm{O} / \mathrm{H}) \sim 8.4$.

In particular the relatively low fraction of GRB hosts with very high oxygen abundances points strongly towards a metallicity dependence in low- $z$ GRBs. In Fig. 18, we compare the metallicity distribution derived from $290.3<z<1$ GRB hosts $(\langle z\rangle=0.6)$ with the expectation from the contribution of field galaxies to the global SFR density at a similar redshift.

As no sufficiently complete sample of field-galaxy metallicities is available in the literature at $z \sim 0.6$, this comparison is based on the stellar-mass function from deep photometric surveys. To derive the red lines in Fig. 18, we start with the double Schechter parameterization of the galaxy-mass function for starforming galaxies at $0.5<z<0.75$ from Tomczak et al. $(2014)^{14}$. Passively evolving galaxies are hence implicitly excluded from this comparison. Using the mean-value of the SFR-stellar mass

\footnotetext{
14 Only subtle differences are introduced in the result when using the mass-function of star-forming galaxies from Ilbert et al. (2013) in a similar redshift interval.
}

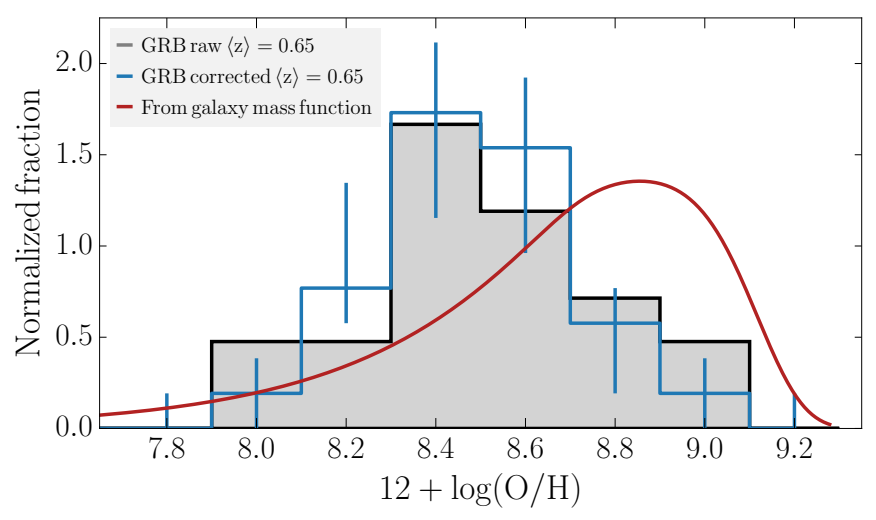

Fig. 18. Blue-solid histogram with error bars: metallicity distribution and its uncertainty of $290.3<z<1$ GRB hosts $(\langle z\rangle=0.65)$. We applied a correction for the over-proportionality of dusty GRBs in the sample (Sects. 2.3.3 and 2.4) and for completeness from the line broadening. Black data show raw measurements. The red line is the expectation from the stellar-mass function at a similar redshift (Tomczak et al. 2014) if GRBs traced star formation without metallicity dependence. Shown curves are normalized to an area of 1 .

relation and its dispersion ${ }^{15}$, we then calculate the $50 \%$ quartile of SFR for a given stellar mass.

Finally, we use the parametrization of Mannucci et al. (2010) to convert the SFR, stellar mass pairs into a metallicity in the same scale as our GRB measurements and weight the resulting galaxy-metallicity function with the SFR ${ }^{16}$.

The fraction of star formation in galaxies above $Z_{\odot}$ can then be obtained via the integral under the red curve of Fig. 18, and is around $50 \%{ }^{17}$. Similarly, Campisi et al. (2011) use N-body simulations with semi-analytical galaxy-formation models to predict around $\sim 50 \%$ of GRBs in galaxies of super-solar metallicity in cases where GRBs traced all star formation equally (their Fig. 4).

Our results therefore directly confirm studies that at low redshift, GRB host properties are affected by a tendency of GRBs to occur in environments with a metallicity below the solar value (e.g., Le Floc'h et al. 2006; Kocevski et al. 2009; Levesque et al. 2010b; Graham \& Fruchter 2013; Perley et al. 2013b, 2015c; Boissier et al. 2013; Vergani et al. 2015; Schulze et al. 2015). The scarcity of high $A_{V}$ and high SFR galaxies as discussed in previous sections further strengthens this interpretation.

The physical driver behind the environmental bias in the GRB production efficiency could in principle relate to either (or both), metallicity or a high stellar mass. Because of the wellstudied impact that metallicity has on stellar evolution, and the lack of an obvious link between GRB formation and a galaxy's stellar mass, we consider a metallicity effect as the most logical explanation for our observations. This interpretation is further

${ }^{15}$ Here, we assume an intrinsic dispersion of 0.3 dex (Speagle et al. 2014) and a slope of 0.8 in the stellar-mass range below $10^{10} M_{\odot}$ (Dunne et al. 2009; Santini et al. 2009; Sobral et al. 2014) and 0.55 above (Whitaker et al. 2012).

${ }^{16}$ The figure and results remain conceptually unchanged if we use the host's $12+\log (\mathrm{O} / \mathrm{H})$ and SFR to derive a stellar mass via Mannucci et al. (2010) to compare it with the SFR-weighted stellar-mass function. 17 Similar values are inferred through the analysis of Perley et al. (2013b), Hunt et al. (2014) and Sobral et al. (2014), which use deep photometric surveys (Kajisawa et al. 2009; Ilbert et al. 2013) to calculate a galaxy stellar mass of $10^{9.7-10.2} M_{\odot}$ at $z \sim 0.6$ above or below which half of all star formation occurs. This implies a metallicity between $Z=0.9 Z_{\odot}$ and $Z=1.4 Z_{\odot}$ in our oxygen-abundance scale (Mannucci et al. 2010). 

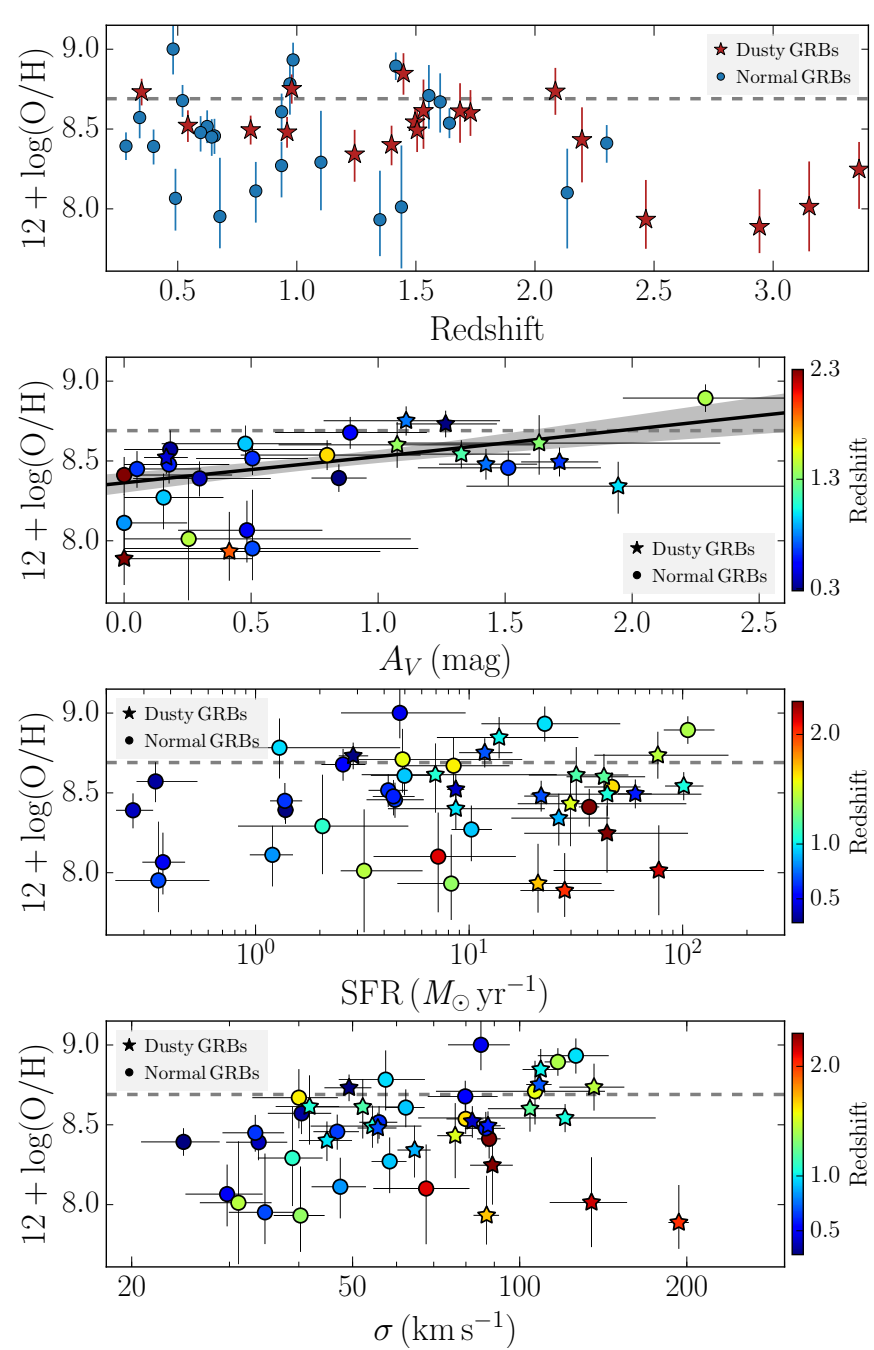

Fig. 19. Gas-phase metallicity as a function of (from top to bottom): redshift, $A_{V}$, SFR, and $\sigma$. Black data and error bars represent individual galaxies. The color coding corresponds to redshift, with the scale given by the color bar. $Z_{\odot}$ is indicated by the horizontal, gray dashed line.

supported by the increasing stellar mass of GRB hosts with increasing redshift (Perley et al. 2015c).

The observed metallicity dependence is unlikely to play a dominant role at $z \gtrsim 3$. Already $z>2$, the metallicity distribution is somewhat shifted towards lower metallicities although the selection biases against low-luminosity hosts become more severe. At $z \sim 3$ galaxies of solar metallicity are scarce even among the most luminous galaxies (Troncoso et al. 2014). Because the metallicity of star-forming galaxies in general is decreasing, GRBs are expected to form also more frequently in the most luminous galaxies. Therefore, we expect GRB hosts at high redshift to represent star-forming galaxies much better than at $z<1$ (see also Greiner et al. 2015a; Perley et al. 2015c).

There is already evidence that the implications of the metallicity dependence are weaker at $z \sim 2$ (Perley et al. 2013b; Schulze et al. 2015), than in $z<1$ GRB-selected galaxies.

\subsubsection{Correlations of metallicity with other host properties}

Figure 19 shows the dependence of host metallicity with redshift, dust reddening, SFR, and emission-line width. A correlation exist ( $p=0.0007$ of all iterations return no dependence)
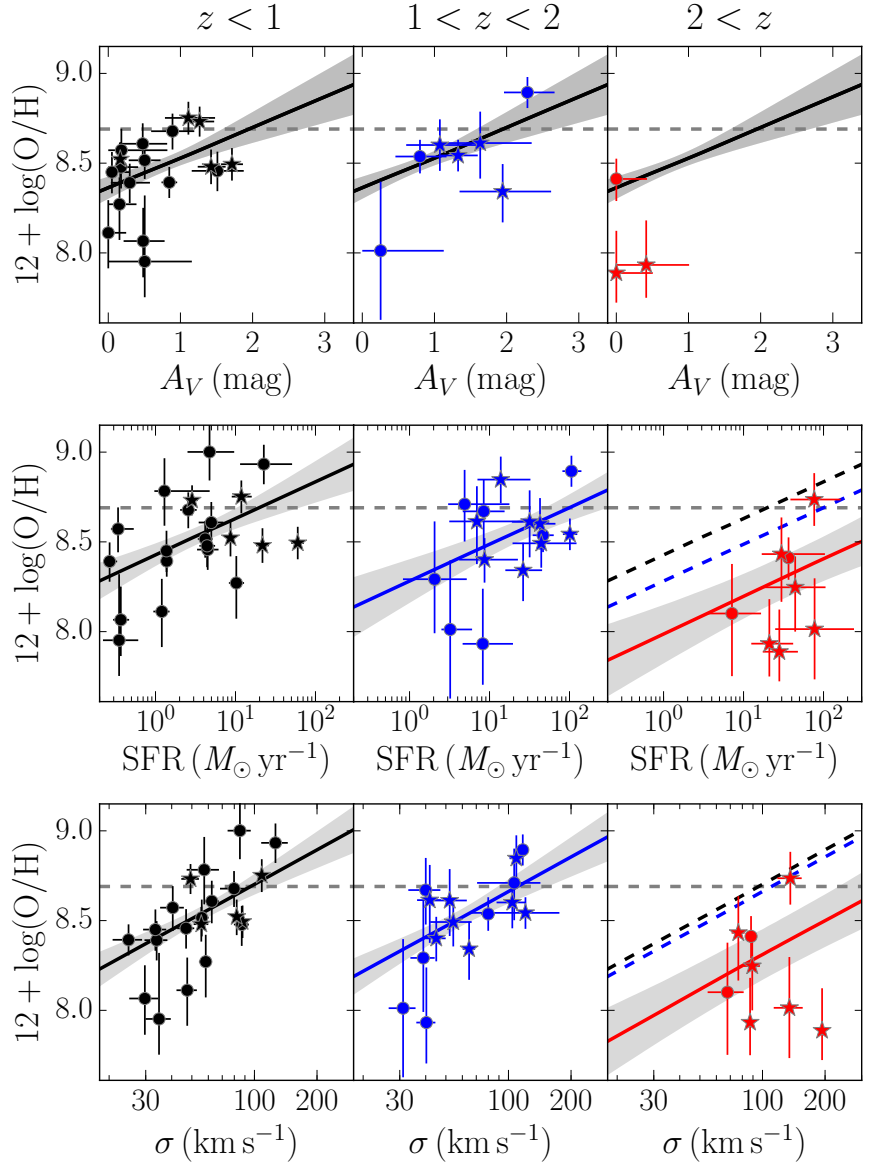

Fig. 20. Gas-phase metallicity as a function of $z$ and (from top to bottom): $A_{V}$, SFR, and $\sigma$ as well as $z<1,1<z<2$, and $2<z$ from left to right. Solid lines denote the best linear regression, with regions of $68 \%$ probability in shaded areas. The last row shows also the relations from the lower-redshift panels in dashed lines. $Z_{\odot}$ is indicated by the horizontal, gray dashed line.

between $A_{V}$ and oxygen abundance similar to $12+\log (\mathrm{O} / \mathrm{H})=$ $8.36+0.17 \cdot A_{V} /(\mathrm{mag})$. This relation is driven by $z<2$ events, as only a few $z>2$ hosts have sufficient data to constrain both $A_{V}$ and $12+\log (\mathrm{O} / \mathrm{H})$ at the same time. At somewhat higher redshift, a correlation between metallicity and dust content is also seen directly along GRB sight-lines as traced by the GRB afterglow and its DLA (Zafar \& Watson 2013).

There are no strong correlations between SFR or $\sigma$ and $12+\log (\mathrm{O} / \mathrm{H})$ in the full host sample, but this is the result of the large redshift range present in Fig. 19. In Fig. 20 we plot oxygen abundance versus $A_{V}$, SFR, and $\sigma$ divided into three redshift bins, $z<1$ with $z_{\text {med }} \sim 0.6,1<z<2$ with $z_{\text {med }} \sim 1.4$, and $2<z$ with $z_{\text {med }} \sim 2.4$. A mild correlation exists between SFR and $12+\log (\mathrm{O} / \mathrm{H})$ similar to $12+\log (\mathrm{O} / \mathrm{H}) \propto \mathrm{SFR}^{0.20 \pm 0.06}$ $(p=0.004)$. Using a common slope, but a different normalization in the three redshift ranges, we fit all data simultaneously and derive

$$
\begin{aligned}
& 12+\log (\mathrm{O} / \mathrm{H})_{z<1}=8.63+0.20 \cdot \log \left(S F R / 10 M_{\odot} \mathrm{yr}^{-1}\right) \\
& 12+\log (\mathrm{O} / \mathrm{H})_{1<z<2}=8.49+0.20 \cdot \log \left(S F R / 10 M_{\odot} \mathrm{yr}^{-1}\right) \\
& 12+\log (\mathrm{O} / \mathrm{H})_{2<z}=8.20+0.20 \cdot \log \left(\mathrm{SFR} / 10 \mathrm{M}_{\odot} \mathrm{yr}^{-1}\right)
\end{aligned}
$$


with uncertainties of 0.05 in the intercept in the earlier two, and 0.11 in the $z>2$ case. The root mean square (rms) dispersion in the $z<1$ and $1<z<2$ redshift range is 0.25 dex each.

The decrease in $\log (\mathrm{O} / \mathrm{H})$ for a given SFR between $z \sim 0.6$ and $z \sim 1.4$ is $0.14 \pm 0.08$ dex, but $0.4 \pm 0.1$ dex for the $z>$ 2 sample (Fig. 20). This is consistent with the relatively slow evolution of the mass-metallicity relation at lower redshift, as well as a more rapid change at $z>1.5$.

The tightest and most significant correlation $(p<0.0001)$ is present between $12+\log (\mathrm{O} / \mathrm{H})$ and $\sigma$ as $12+\log (\mathrm{O} / \mathrm{H}) \propto$ $\sigma^{0.64 \pm 0.16}$ at $z<2$. We again fit all data together with a common slope and free normalization in the three redshift intervals and obtain for $\sigma$

$$
\begin{aligned}
& 12+\log (\mathrm{O} / \mathrm{H})_{z<1}=8.70+0.64 \cdot \log \left(\sigma / 100 \mathrm{~km} \mathrm{~s}^{-1}\right) \\
& 12+\log (\mathrm{O} / \mathrm{H})_{1<z<2}=8.66+0.64 \cdot \log \left(\sigma / 100 \mathrm{~km} \mathrm{~s}^{-1}\right) \\
& 12+\log (\mathrm{O} / \mathrm{H})_{2<z}=8.31+0.64 \cdot \log \left(\sigma / 100 \mathrm{~km} \mathrm{~s}^{-1}\right)
\end{aligned}
$$

with uncertainties of $0.05 / 0.06$ in the intercept in the earlier two, and 0.12 in the $z>2$ case. The behavior is qualitatively very similar to the relation between SFR and oxygen abundance but with a lower scatter, i.e., a tighter correlation.

Low- and medium-redshift samples return comparable bestfit relations. In both cases $\sigma=100 \mathrm{~km} \mathrm{~s}^{-1}$ roughly corresponds to a metallicity of $Z \approx Z_{\odot}$. At $z>2$, this value shifts downward by $0.4 \pm 0.1$ dex to an oxygen abundance of $12+\log (\mathrm{O} / \mathrm{H}) \approx 8.3$, but is affected by large measurement uncertainties. The rms dispersion in the $z<1$ and $1<z<2$ redshift range is only 0.19 dex and $0.20 \mathrm{dex}$, respectively. Given that the typical error in the metallicity measurement via the strong-line diagnostic is already $0.11 / 0.16 \mathrm{dex}$, this implies that the very simple measurement of $\sigma$ provides already a good estimate of the gas-phase metallicity.

Taken at face value, there is no statistically significant correlation in the highest-redshift bin $(z>2)$ between SFR and $12+\log (\mathrm{O} / \mathrm{H})$ as well as $\sigma$ and $12+\log (\mathrm{O} / \mathrm{H})$. Because of the large measurement uncertainties however, the data are consistent within errors with the lower-redshift slope, but, as argued above, a lower normalization.

\section{Summary}

We present data and initial results from X-Shooter emissionline spectroscopy of star-forming galaxies selected through long GRBs in a redshift range $0.1<z<3$.6. In total, we discuss observations of 96 individual targets which allow us to trace the physical properties of GRB hosts through cosmic time. The galaxies have a median redshift of $z_{\text {med }}=1.6$ and most of them are in the range $0.5<z<1.5(35 \%)$ and $1.5<z<2.5(41 \%)$. We carefully test for selection effects in the sample, and statistically correct for a somewhat higher fraction of dusty GRBs $(35 \pm 5 \%)$ when compared to the one of representative GRB samples $(20-30 \%)$.

Our observation yield 16 new GRB redshifts, which increase the redshift completeness of previous samples to $87 \%$ (TOUGH) and $97 \%$ (BAT6), with median redshifts of $z_{\mathrm{med}}=2.06 \pm 0.18$ and $z_{\text {med }}=1.67 \pm 0.15$, respectively.

After excluding a very nearby event (GRB 100316D), a possibly short GRB (GRB 100816A), and five galaxies without clear emission lines we arrive at a total number of 89 emissionline spectra from galaxies selected by GRBs. Based on these data, we provide new scaling relations to derive SFRs from emission lines of [O II] $\mathrm{H} \beta$, and [O III] even in the absence of a reliable dust correction (but with a large scatter). We measure galaxy-integrated star formation rates (SFRs), visual dustattenuations $\left(A_{V}\right)$, line broadening $(\sigma)$, and oxygen abundances $(12+\log (\mathrm{O} / \mathrm{H}))$ for our targets (Table 4$)$. This yields the largest and most comprehensive sample of emission-line spectroscopy of GRB hosts available to date and allows us to study the distribution of the physical parameters and their evolution with redshift.

Our main results can be summarized as follows:

1. The distribution of $A_{V}$ for long GRB hosts is broad with the bulk of the targets at $A_{V}<1.5 \mathrm{mag}$ and median $A_{V}^{\text {med }} \sim$ $0.75 \mathrm{mag}$ at $z<2$. We observe a mild trend with redshift such that GRB hosts at $z=1.5$ tend to have larger dust content $\left(A_{V}^{\text {med }} \sim 1.2 \mathrm{mag}\right)$ and line broadening $\left(\sigma_{\text {med }} \sim 70 \mathrm{kms}\right)$ on average than low-redshift hosts $\left(A_{V}^{\text {med }} \sim 0.6 \mathrm{mag}\right.$, and $\left.\sigma_{\text {med }} \sim 40 \mathrm{kms}\right)$. The significance of each of these results is only around $2 \sigma$, but the evolution in $A_{V}$ is remarkably consistent with a similar behavior observed for GRB afterglows (Covino et al. 2013).

2. There is a strong evolution of the average GRB host properties with redshift. The median SFR evolves from $S F R_{\text {med }}=$ $0.6 M_{\odot} \mathrm{yr}^{-1}$ at $z \sim 0.6$ up to $S F R_{\text {med }}=15 M_{\odot} \mathrm{yr}^{-1}$ at $z \sim 2$ above which it does not increase significantly any further.

3. The ratio of $[\mathrm{O} \mathrm{III}] /[\mathrm{O} \mathrm{II}]$ of the galaxies increases with redshift as well, which moves the star-forming galaxies away from the location of local galaxies in the BPT diagram. The value changes from $[\mathrm{O} \mathrm{III]} /[\mathrm{O} \mathrm{II}] \sim 0.9$ at $z \sim 0.3$ by a factor of three to $[\mathrm{O} \mathrm{III}] /[\mathrm{O}$ II] $\sim 3$ at $z \sim 3$. While roughly similar at $z \sim 0.6$ and $z \sim 1.4$, the metallicity distribution of GRB hosts starts to shift to lower metallicities above $z=2$.

4. We find a strong correlation between $\sigma$ and SFR broadly consistent with $S F R \propto \sigma^{4}$ as known from the local FaberJackson relation. There is no statistically significant evidence in the data that this proportionality evolves with redshift.

5. Further correlations exist between oxygen abundance and visual attenuation $\left(12+\log (\mathrm{O} / \mathrm{H}) \propto 0.17 \cdot A_{V}\right)$, SFR $(12+$ $\left.\log (\mathrm{O} / \mathrm{H}) \propto S F R^{0.2}\right)$, and $\sigma\left(12+\log (\mathrm{O} / \mathrm{H}) \propto \sigma^{0.6}\right)$. In the last two cases, we observe a relatively similar behavior at $z<1$ and $1<z<2$, but an evolution towards lower metallicities at $z>2$ by $\sim 0.4$ dex for a given SFR or $\sigma$.

6. We detect several hosts with high oxygen abundances which rule out a strict metallicity cutoff for GRB hosts below the solar values (in the adopted scale). At the same time, however, the fraction of GRBs hosted in $Z>Z_{\odot}$ galaxies at $z<1$ is smaller $(18 \pm 7 \%)$ than what would be expected from the contribution of similarly metal-rich galaxies to the total cosmic SFR ( $\gtrsim 50 \%)$. A mechanisms is thus at place that quenches GRB formation at the highest metallicities.

At $z=3$, most of the star formation takes place in galaxies of $Z \lesssim 0.5 Z_{\odot}$. At the same time GRB hosts with similar metallicities are relatively common in our sample at lower redshift. Under the conditions that the probability of producing GRBs below a certain metallicity is constant (and not a strong function of metallicity itself) then by $z \sim 3$ GRBs host will probe a large fraction of the total star formation. In absence of further secondary environmental factors, GRB hosts would then provide an extensive picture of high-redshift, star-forming galaxies. This conclusion is similar to the one reached through studying metallicities of GRB-DLAs (Fynbo et al. 2008; Arabsalmani et al. 2015), the UV-luminosity function at a similar redshift (Jakobsson et al. 2005; Schulze et al. 2015; Greiner et al. 2015a), cosmological simulations (Chisari et al. 2010) or modeling the mass distribution of GRB hosts (Kocevski et al. 2009). 
Our results can be understood in the cosmological context and a picture drawn by recent studies (Modjaz et al. 2008; Kocevski et al. 2009; Boissier et al. 2013; Perley et al. 2013b; Vergani et al. 2015; Wang \& Dai 2014; Trenti et al. 2015; Perley et al. 2015c; Schulze et al. 2015) in which the GRB host properties at lower redshift $(z<1-2)$ are driven by the GRB's tendency to occur in lower-metallicity galaxies without fully avoiding metal-rich ones. The scarcity of high metallicity, high $A_{V}$, and high SFR galaxies at low redshift strongly supports this interpretation.

Acknowledgements. We are grateful to the referee and the editor, Rubina Kotak, for comments and for providing an extensive and constructive report, which has helped to increase the quality and strength of the manuscript significantly. We thank the Swift team for building and operating such an excellent facility. We also acknowledge the astronomical and technical support at Paranal observatory without which none of the presented spectra would have been taken. The Dark Cosmology Centre is funded by the Danish National Research Foundation. The research leading to these results has received funding from the European Research Council under the European Union's Seventh Framework Program (FP7/2007-2013)/ERC Grant agreement No. EGGS-278202. Part of the funding for GROND (both hardware as well as personnel) was generously granted from the Leibniz-Prize to Prof. G. Hasinger (DFG grant HA 1850/281). The data presented here were obtained in part with ALFOSC, which is provided by the Instituto de Astrofisica de Andalucia (IAA) under a joint agreement with the University of Copenhagen and NOTSA. T.K. thanks A. Müller, L. Guzman, and L. Watson for providing the vibrant scientific atmosphere in Vitacura where some of this work was carried out. D.M. acknowledges the Instrument center for Danish astrophysics (IDA) for support. S.Sch. acknowledges support from CONICYT-Chile FONDECYT 3140534, Basal- CATA PFB06/2007, and Project IC120009 "Millennium Institute of Astrophysics (MAS)" of Iniciativa Cientifica Milenio del Ministerio de Economia, Fomento y Turismo. A.R. acknowledges support from PRIN-INAF 2012/13. Support for DAP was provided by NASA through Hubble Fellowship grant HST-HF-51296.01-A awarded by the Space Telescope Science Institute (STScI), which is operated by the Association of Universities for Research in Astronomy (AURA), Inc., for NASA, under contract NAS 5-26555, and by an award issued by JPL/Caltech. E.P. acknowledges support from grants ASI-INAF I/088/06/0 and PRIN INAF 2011. P.S. acknowledges support through the Sofja Kovalevskaja Award from the Alexander von Humboldt Foundation of Germany. J.F.G. acknowledges support through the Sofja Kovalevskaja Award to P. Schady from the Alexander von Humboldt Foundation of Germany. S.K. and A.N.G. acknowledge support by DFG grant K1 766/16-1. S.Sav. acknowledges support from the Bundesministerium für Wirtschaft and Technologie through DLR (Deutsches Zentrum für Luft- und Raumfahrt e.V.) FKZ 50 OR 1211. Based in part on observations collected with the $2.2 \mathrm{~m}$ MPG telescope on La Silla as part of the program CN2014B-102.

\section{References}

Abazajian, K. N., Adelman-McCarthy, J. K., Agüeros, M. A., et al. 2009, ApJS, 182,543

Ackermann, M., Ajello, M., Asano, K., et al. 2014, Science, 343, 42

Aihara, H., Allende Prieto, C., An, D., et al. 2011, ApJS, 193, 29

Alloin, D., Collin-Souffrin, S., Joly, M., \& Vigroux, L. 1979, A\&A, 78, 200

Appenzeller, I., Fricke, K., Fürtig, W., et al. 1998, The Messenger, 94, 1

Arabsalmani, M., Møller, P., Fynbo, J. P. U., et al. 2015, MNRAS, 446, 990

Arnouts, S., Cristiani, S., Moscardini, L., et al. 1999, MNRAS, 310, 540

Asplund, M., Grevesse, N., Sauval, A. J., \& Scott, P. 2009, ARA\&A, 47, 481

Baldwin, J. A., Phillips, M. M., \& Terlevich, R. 1981, PASP, 93, 5

Berger, E., Fox, D. B., Kulkarni, S. R., Frail, D. A., \& Djorgovski, S. G. 2007,

ApJ, 660, 504

Bloom, J. S., Kulkarni, S. R., \& Djorgovski, S. G. 2002, AJ, 123, 111

Boissier, S., Salvaterra, R., Le Floc'h, E., et al. 2013, A\&A, 557, A34

Brinchmann, J., Pettini, M., \& Charlot, S. 2008, MNRAS, 385, 769

Bufano, F., Pian, E., Sollerman, J., et al. 2012, ApJ, 753, 67

Burrows, D. N., Hill, J. E., Nousek, J. A., et al. 2005, Space Sci. Rev., 120, 165

Butler, N., Watson, A. M., Kutyrev, A., et al. 2014, GCN, 15732

Campisi, M. A., Tapparello, C., Salvaterra, R., Mannucci, F., \& Colpi, M. 2011,

MNRAS, 417, 1013

Cano, Z., Xu, D., Malesani, D., Jakobsson, P., \& Pursimo, T. 2014, GCN, 15743

Capak, P., Cowie, L. L., Hu, E. M., et al. 2004, AJ, 127, 180

Cenko, S. B., Kelemen, J., Harrison, F. A., et al. 2009, ApJ, 693, 1484

Chabrier, G. 2003, PASP, 115, 763

Chary, R., Berger, E., \& Cowie, L. 2007, ApJ, 671, 272

Chen, H.-W. 2012, MNRAS, 419, 3039
Chen, H., Perley, D. A., Pollack, L. K., et al. 2009, ApJ, 691, 152 Chisari, N. E., Tissera, P. B., \& Pellizza, L. J. 2010, MNRAS, 408, 647 Christensen, L., Hjorth, J., \& Gorosabel, J. 2004, A\&A, 425, 913 Christensen, L., Laursen, P., Richard, J., et al. 2012, MNRAS, 427, 1973 Covino, S., Melandri, A., Salvaterra, R., et al. 2013, MNRAS, 432, 1231 Cucchiara, A., Fumagalli, M., Rafelski, M., et al. 2015, ApJ, 804, 51 Cucciati, O., Tresse, L., Ilbert, O., et al. 2012, A\&A, 539, A31 de Ugarte Postigo, A., Thöne, C. C., Goldoni, P., Fynbo, J. P. U., \& X-Shooter GRB Collaboration 2011, Astron. Nachr., 332, 297

de Ugarte Postigo, A., Campana, S., Thöne, C. C., et al. 2013, A\&A, 557, L18 de Ugarte Postigo, A., Thöne, C. C., Rowlinson, A., et al. 2014, A\&A, 563, A62 Dunne, L., Ivison, R. J., Maddox, S., et al. 2009, MNRAS, 394, 3 Elíasdóttir, Á., Fynbo, J. P. U., Hjorth, J., et al. 2009, ApJ, 697, 1725 Elliott, J., Greiner, J., Khochfar, S., et al. 2012, A\&A, 539, A113 Elliott, J., Krühler, T., Greiner, J., et al. 2013, A\&A, 556, A23 Erb, D. K., Shapley, A. E., Pettini, M., et al. 2006a, ApJ, 644, 813 Erb, D. K., Steidel, C. C., Shapley, A. E., et al. 2006b, ApJ, 646, 107 Faber, S. M., \& Jackson, R. E. 1976, ApJ, 204, 668

Filgas, R., Krühler, T., Greiner, J., et al. 2011, A\&A, 526, A113 Förster Schreiber, N. M., Genzel, R., Bouché, N., et al. 2009, ApJ, 706, 1364 Friis, M., De Cia, A., Krühler, T., et al. 2015, MNRAS, 451, 167 Fruchter, A. S., Levan, A. J., Strolger, L., et al. 2006, Nature, 441, 463 Fynbo, J. P. U., Jakobsson, P., Möller, P., et al. 2003, A\&A, 406, L63 Fynbo, J. P. U., Starling, R. L. C., Ledoux, C., et al. 2006, A\&A, 451, L47 Fynbo, J. P. U., Prochaska, J. X., Sommer-Larsen, J., Dessauges-Zavadsky, M., \& Møller, P. 2008, ApJ, 683, 321

Fynbo, J. P. U., Krühler, T., Leighly, K., et al. 2014, A\&A, 572, A12 Galama, T. J., Vreeswijk, P. M., van Paradijs, J., et al. 1998, Nature, 395, 670 Gawiser, E., van Dokkum, P. G., Herrera, D., et al. 2006, ApJS, 162, 1 Gehrels, N., Chincarini, G., Giommi, P., et al. 2004, ApJ, 611, 1005 Gehrels, N., Ramirez-Ruiz, E., \& Fox, D. B. 2009, ARA\&A, 47, 567 Gendre, B., Stratta, G., Atteia, J. L., et al. 2013, ApJ, 766, 30 Gil de Paz, A., Madore, B. F., \& Pevunova, O. 2003, ApJS, 147, 29 Gilbank, D. G., Baldry, I. K., Balogh, M. L., Glazebrook, K., \& Bower, R. G. 2010, MNRAS, 405, 2594

Goad, M. R., Osborne, J. P., Beardmore, A. P., \& Evans, P. A. 2012, GCN, 12922 Goldoni, P., Royer, F., François, P., et al. 2006, in SPIE Conf. Ser., 6269 Gorosabel, J., Pérez-Ramírez, D., Sollerman, J., et al. 2005, A\&A, 444, 711 Gorosabel, J., Huelamo, N., Sanchez-Ramirez, R., et al. 2012, GCN, 13591 Graham, J. F., \& Fruchter, A. S. 2013, ApJ, 774, 119

Greiner, J., Bornemann, W., Clemens, C., et al. 2008, PASP, 120, 405 Greiner, J., Krühler, T., Klose, S., et al. 2011, A\&A, 526, A30 Greiner, J., Krühler, T., Nardini, M., et al. 2013, A\&A, 560, A70 Greiner, J., Yu, H.-F., Krühler, T., et al. 2014, A\&A, 568, A75 Greiner, J., Fox, D. B., Schady, P., et al. 2015a, ApJ, 809, 76 Greiner, J., Mazzali, P. A., Kann, D. A., et al. 2015b, Nature, 523, 189 Groot, P. J., Galama, T. J., van Paradijs, J., et al. 1998, ApJ, 493, L27

Guetta, D., \& Della Valle, M. 2007, ApJ, 657, L73 Guidorzi, C., \& Mundell, C. G. 2012, GCN, 13651 Hirschi, R., Meynet, G., \& Maeder, A. 2005, A\&A, 443, 581

Hjorth, J., Sollerman, J., Møller, P., et al. 2003, Nature, 423, 847 Hjorth, J., Malesani, D., Jakobsson, P., et al. 2012, ApJ, 756, 187 Hunt, L., Palazzi, E., Rossi, A., et al. 2011, ApJ, 736, L36

Hunt, L. K., Palazzi, E., Michałowski, M. J., et al. 2014, A\&A, 565, A112 Ilbert, O., Arnouts, S., McCracken, H. J., et al. 2006, A\&A, 457, 841

Ilbert, O., McCracken, H. J., Le Fèvre, O., et al. 2013, A\&A, 556, A55 Jakobsson, P., Hjorth, J., Fynbo, J. P. U., et al. 2004, ApJ, 617, L21 Jakobsson, P., Björnsson, G., Fynbo, J. P. U., et al. 2005, MNRAS, 362, 245

Jakobsson, P., Hjorth, J., Malesani, D., et al. 2012, ApJ, 752, 62

Jaunsen, A. O., Rol, E., Watson, D. J., et al. 2008, ApJ, 681, 453

Kajisawa, M., Ichikawa, T., Tanaka, I., et al. 2009, ApJ, 702, 1393

Kann, D. A., Schady, P., Olivares E., F., et al. 2015, A\&A, submitted

Kauffmann, G., Heckman, T. M., Tremonti, C., et al. 2003, MNRAS, 346, 1055 Kelly, P. L., Filippenko, A. V., Modjaz, M., \& Kocevski, D. 2014, ApJ, 789, 23 Kennicutt, Jr., R. C. 1998, ARA\&A, 36, 189

Kewley, L. J., \& Dopita, M. A. 2002, ApJS, 142, 35

Kewley, L. J., \& Ellison, S. L. 2008, ApJ, 681, 1183

Kewley, L. J., Dopita, M. A., Sutherland, R. S., Heisler, C. A., \& Trevena, J. 2001, ApJ, 556, 121

Kewley, L. J., Geller, M. J., \& Jansen, R. A. 2004, AJ, 127, 2002

Kewley, L. J., Dopita, M. A., Leitherer, C., et al. 2013a, ApJ, 774, 100

Kewley, L. J., Maier, C., Yabe, K., et al. 2013b, ApJ, 774, L10

Kissler-Patig, M., Pirard, J.-F., Casali, M., et al. 2008, A\&A, 491, 941

Kistler, M. D., Yüksel, H., Beacom, J. F., Hopkins, A. M., \& Wyithe, J. S. B. 2009, ApJ, 705, L104

Kocevski, D., \& West, A. A. 2011, ApJ, 735, L8

Kocevski, D., West, A. A., \& Modjaz, M. 2009, ApJ, 702, 377

Kriek, M., van Dokkum, P. G., Franx, M., et al. 2007, ApJ, 669, 776 
Krühler, T., Küpcü Yoldas, A., Greiner, J., et al. 2008, ApJ, 685, 376 Krühler, T., Greiner, J., Schady, P., et al. 2011, A\&A, 534, A108 Krühler, T., Fynbo, J. P. U., Geier, S., et al. 2012a, A\&A, 546, A8 Krühler, T., Malesani, D., Milvang-Jensen, B., et al. 2012b, ApJ, 758, 46 Krühler, T., Nicuesa Guelbenzu, A., \& Greiner, J. 2012c, GCN, 14049 Krühler, T., Ledoux, C., Fynbo, J. P. U., et al. 2013, A\&A, 557, A18 Kumar, P., \& Zhang, B. 2015, Phys. Rep., 561, 1 Lamb, D. Q., \& Reichart, D. E. 2000, ApJ, 536, 1

Laskar, T., Berger, E., \& Chary, R.-R. 2011, ApJ, 739, Le Floc'h, E., Duc, P.-A., Mirabel, I. F., et al. 2003, A\&A, 400, 499 Le Floc'h, E., Charmandaris, V., Forrest, W. J., et al. 2006, ApJ, 642, 636 Ledoux, C., Vreeswijk, P. M., Smette, A., et al. 2009, A\&A, 506, 661 Leloudas, G., Schulze, S., Krühler, T., et al. 2015, MNRAS, 449, 917 Levan, A., Fruchter, A., Rhoads, J., et al. 2006, ApJ, 647, 471 Levan, A. J., Tanvir, N. R., Fruchter, A. S., et al. 2014a, ApJ, 792, 115 Levan, A. J., Tanvir, N. R., Starling, R. L. C., et al. 2014b, ApJ, 781, 13 Levesque, E. M. 2014, PASP, 126, 1

Levesque, E. M., Berger, E., Kewley, L. J., \& Bagley, M. M. 2010a, AJ, 139, 694

Levesque, E. M., Kewley, L. J., Berger, E., \& Zahid, H. J. 2010b, AJ, 140, 1557 Levesque, E. M., Kewley, L. J., Graham, J. F., \& Fruchter, A. S. 2010c, ApJ, 712, L26

Levesque, E. M., Berger, E., Soderberg, A. M., \& Chornock, R. 2011, ApJ, 739, 23

Littlejohns, O. M., Butler, N. R., Cucchiara, A., et al. 2015, MNRAS, 449, 2919 Ly, C., Lee, J. C., Dale, D. A., et al. 2011, ApJ, 726, 109 MacFadyen, A. I., \& Woosley, S. E. 1999, ApJ, 524, 262

Maiolino, R., Nagao, T., Grazian, A., et al. 2008, A\&A, 488, 463 Malesani, D., Krühler, T., de Ugarte Postigo, A., et al. 2012, GCN, 13639

Mannucci, F., Cresci, G., Maiolino, R., Marconi, A., \& Gnerucci, A. 2010, MNRAS, 408, 2115

Mannucci, F., Salvaterra, R., \& Campisi, M. A. 2011, MNRAS, 414, 1263

Maselli, A., Barthelmy, S. D., Baumgartner, W. H., et al. 2012, GCN, 14045

McBreen, S., Krühler, T., Rau, A., et al. 2010, A\&A, 516, A71

McGaugh, S. S. 1991, ApJ, 380, 140

Melandri, A., Sbarufatti, B., D’ Avanzo, P., et al. 2012, MNRAS, 421, 1265

Melandri, A., Pian, E., D’Elia, V., et al. 2014, A\&A, 567, A29

Michałowski, M. J., Kamble, A., Hjorth, J., et al. 2012, ApJ, 755, 85

Milvang-Jensen, B., Fynbo, J. P. U., Malesani, D., et al. 2012, ApJ, 756, 25

Modigliani, A., Goldoni, P., Royer, F., et al. 2010, in SPIE Conf. Ser., 7737

Modjaz, M., Kewley, L., Kirshner, R. P., et al. 2008, AJ, 135, 1136

Morgan, A. N., Perley, D. A., Cenko, S. B., et al. 2014, MNRAS, 440, 1810

Murray, N., Quataert, E., \& Thompson, T. A. 2005, ApJ, 618, 569

Nagao, T., Maiolino, R., \& Marconi, A. 2006, A\&A, 459, 85

Nakauchi, D., Kashiyama, K., Suwa, Y., \& Nakamura, T. 2013, ApJ, 778, 67

Nicuesa, A., Kruehler, T., \& Greiner, J. 2010, GCN, 10835

Niino, Y., Hashimoto, T., Aoki, K., et al. 2012, PASJ, 64, 115

Noll, S., Kausch, W., Barden, M., et al. 2012, A\&A, 543, A92

Oates, S. R., Markwardt, C. B., Norris, J., Evans, P. A., \& Littlejohns, O. 2010 GCN Report, 300

Oke, J. B., Cohen, J. G., Carr, M., et al. 1995, PASP, 107, 375

Osterbrock, D. E. 1989, Astrophysics of gaseous nebulae and active galactic nuclei (University Science Books)

Ovaldsen, J.-E., Jaunsen, A. O., Fynbo, J. P. U., et al. 2007, ApJ, 662, 294

Pagel, B. E. J., Edmunds, M. G., Blackwell, D. E., Chun, M. S., \& Smith, G. 1979, MNRAS, 189, 95

Pei, Y. C. 1992, ApJ, 395, 130

Perley, D. A., Cenko, S. B., Bloom, J. S., et al. 2009, AJ, 138, 1690

Perley, D. A., Cenko, S. B., Morgan, A. N., \& Krühler, T. 2012, GCN, 14056

Perley, D. A., Bloom, J. S., \& Prochaska, J. X. 2013a, in EAS Publ. Ser. 61, eds. A. J. Castro-Tirado, J. Gorosabel, \& I. H. Park, 391

Perley, D. A., Levan, A. J., Tanvir, N. R., et al. 2013b, ApJ, 778, 128

Perley, D. A., Cenko, S. B., Corsi, A., et al. 2014, ApJ, 781, 37

Perley, D. A., Krühler, T., Schulze, S., et al. 2015a, ApJ, submitted [arXiv: 1504.02482]

Perley, D. A., Perley, R. A., Hjorth, J., et al. 2015b, ApJ, 801, 102

Perley, D. A., Tanvir, N. R., Hjorth, J., et al. 2015c, ApJ, submitted [arXiv: 1504.02479]

Pettini, M., \& Pagel, B. E. J. 2004, MNRAS, 348, L59

Pettini, M., Shapley, A. E., Steidel, C. C., et al. 2001, ApJ, 554, 981

Pian, E., Mazzali, P. A., Masetti, N., et al. 2006, Nature, 442, 1011

Piranomonte, S., Vergani, S. D., Malesani, D., et al. 2011, GCN, 12164

Piranomonte, T., Japelj, J., Vergani, S.-D., et al. 2015, MNRAS, 452, 3293

Planck Collaboration XVI. 2014, A\&A, 571, A16

Podsiadlowski, P., Mazzali, P. A., Nomoto, K., Lazzati, D., \& Cappellaro, E. 2004, ApJ, 607, L17

Prochaska, J. X., Bloom, J. S., Chen, H.-W., et al. 2004, ApJ, 611, 200
Prochaska, J. X., Chen, H.-W., Dessauges-Zavadsky, M., \& Bloom, J. S. 2007, ApJ, 666, 267

Prochaska, J. X., Sheffer, Y., Perley, D. A., et al. 2009, ApJ, 691, L27

Racusin, J. L., Barthelmy, S. D., Beardmore, A. P., et al. 2011, GCN, 12600

Reddy, N. A., \& Steidel, C. C. 2009, ApJ, 692, 778

Richard, J., Jones, T., Ellis, R., et al. 2011, MNRAS, 413, 643

Robertson, B. E., \& Ellis, R. S. 2012, ApJ, 744, 95

Rossi, A., Nicuesa Guelbenzu, A., \& Greiner, J. 2011, GCN, 12605

Rossi, A., Klose, S., Ferrero, P., et al. 2012, A\&A, 545, A77

Rossi, A., Piranomonte, S., Savaglio, S., et al. 2014, A\&A, 572, A47

Salvaterra, R., Campana, S., Vergani, S. D., et al. 2012, ApJ, 749, 68

Santini, P., Fontana, A., Grazian, A., et al. 2009, A\&A, 504, 751

Savaglio, S. 2006, New J. Phys., 8, 195

Savaglio, S., Glazebrook, K., Le Borgne, D., et al. 2005, ApJ, 635, 260

Savaglio, S., Glazebrook, K., \& Le Borgne, D. 2009, ApJ, 691, 182

Saxton, C. J., Cummings, J. R., Kuin, N. P. M., et al. 2012, GCN, 12980

Schady, P., Savaglio, S., Müller, T., et al. 2014, A\&A, 570, A52

Schady, P., Kruehler, T., Greiner, J., et al. 2015, A\&A, 579, 126

Schlafly, E. F., \& Finkbeiner, D. P. 2011, ApJ, 737, 103

Schulze, S., Malesani, D., Cucchiara, A., et al. 2014, A\&A, 566, A102

Schulze, S., Chapman, R., Hjorth, J., et al. 2015, ApJ, 808, 73

Shapley, A. E., Coil, A. L., Ma, C.-P., \& Bundy, K. 2005, ApJ, 635, 1006

Sobral, D., Best, P. N., Smail, I., et al. 2014, MNRAS, 437, 3516

Soderberg, A. M., Nakar, E., Cenko, S. B., et al. 2007, ApJ, 661, 982

Sollerman, J., Östlin, G., Fynbo, J. P. U., et al. 2005, New Astron., 11, 103

Sonbas, E., Baumgartner, W. H., Burrows, D. N., et al. 2012, GCN, 12920

Sparre, M., Sollerman, J., Fynbo, J. P. U., et al. 2011, ApJ, 735, L24

Speagle, J. S., Steinhardt, C. L., Capak, P. L., \& Silverman, J. D. 2014, ApJS, 214,15

Stanek, K. Z., Gnedin, O. Y., Beacom, J. F., et al. 2006, Acta Astron., 56, 333

Starling, R. L. C. Wiersema, K., Levan, A. J., et al. 2011, MNRAS, 411, 2792

Steidel, C. C., Giavalisco, M., Pettini, M., Dickinson, M., \& Adelberger, K. L. 1996, ApJ, 462, L17

Steidel, C. C., Rudie, G. C., Strom, A. L., et al. 2014, ApJ, 795, 165

Storey, P. J., \& Zeippen, C. J. 2000, MNRAS, 312, 813

Svensson, K. M., Levan, A. J., Tanvir, N. R., Fruchter, A. S., \& Strolger, L.-G. 2010, MNRAS, 405, 57

Svensson, K. M., Levan, A. J., Tanvir, N. R., et al. 2012, MNRAS, 421, 25

Tanvir, N. R., Barnard, V. E., Blain, A. W., et al. 2004, MNRAS, 352, 1073

Tanvir, N. R., Levan, A. J., Rol, E., et al. 2008, MNRAS, 388, 1743

Tanvir, N. R., Levan, A. J., Fruchter, A. S., et al. 2012, ApJ, 754, 46

Thöne, C. C., Fynbo, J. P. U., Goldoni, P., et al. 2013, MNRAS, 428, 3590

Tody, D. 1993, in Astronomical Data Analysis Software and Systems II, ASP Conf. Ser., 52, 173

Tomczak, A. R., Quadri, R. F., Tran, K.-V. H., et al. 2014, ApJ, 783, 85

Tremonti, C. A., Heckman, T. M., Kauffmann, G., et al. 2004, ApJ, 613, 898

Trenti, M., Perna, R., Levesque, E. M., Shull, J. M., \& Stocke, J. T. 2012, ApJ, 749, L38

Trenti, M., Perna, R., \& Jimenez, R. 2015, ApJ, 802, 103

Troja, E., Barthelmy, S. D., Beardmore, A. P., et al. 2012, GCN, 13588

Troja, E., Beardmore, A. P., Bernardini, M. G., et al. 2014, GCN, 15728

Troncoso, P., Maiolino, R., Sommariva, V., et al. 2014, A\&A, 563, A58

van der Horst, A. J., Kouveliotou, C., Gehrels, N., et al. 2009, ApJ, 699, 1087

van der Horst, A. J., Paragi, Z., de Bruyn, A. G., et al. 2014, MNRAS, 444, 3151

Vergani, S. D., Flores, H., Covino, S., et al. 2011, A\&A, 535, A127

Vergani, S. D., Salvaterra, R., Japelj, J., et al. 2015, A\&A, 581, A102

Vernet, J., Dekker, H., D'Odorico, S., et al. 2011, A\&A, 536, A105

Virgili, F. J., Mundell, C. G., Pal'shin, V., et al. 2013, ApJ, 778, 54

Vreeswijk, P. M., Fruchter, A., Kaper, L., et al. 2001, ApJ, 546, 672

Vreeswijk, P. M., Ellison, S. L., Ledoux, C., et al. 2004, A\&A, 419, 927

Vreeswijk, P. M., Ledoux, C., Smette, A., et al. 2007, A\&A, 468, 83

Wang, F. Y., \& Dai, Z. G. 2014, ApJS, 213, 15

Whitaker, K. E., van Dokkum, P. G., Brammer, G., \& Franx, M. 2012, ApJ, 754 L29

Wiersema, K. 2011, MNRAS, 414, 2793

Wiersema, K., Curran, P. A., Krühler, T., et al. 2012a, MNRAS, 426, 2

Wiersema, K., Goldoni, P., Fynbo, J. P. U., et al. 2012b, GCN, 12991

Wijers, R. A. M. J., Bloom, J. S., Bagla, J. S., \& Natarajan, P. 1998, MNRAS, 294, L13

Wolfe, A. M., Gawiser, E., \& Prochaska, J. X. 2005, ARA\&A, 43, 861

Woosley, S. E. 1993, ApJ, 405, 273

Wuyts, S., Förster Schreiber, N. M., van der Wel, A., et al. 2011, ApJ, 742, 96

Xu, D., de Ugarte Postigo, A., Leloudas, G., et al. 2013, ApJ, 776, 98

Yoon, S.-C., Langer, N., \& Norman, C. 2006, A\&A, 460, 199

Zafar, T., \& Watson, D. 2013, A\&A, 560, A26

Zafar, T., Watson, D., Fynbo, J. P. U., et al. 2011, A\&A, 532, A143

Zahid, H. J., Kewley, L. J., \& Bresolin, F. 2011, ApJ, 730, 137

Zahid, H. J., Kashino, D., Silverman, J. D., et al. 2014, ApJ, 792, 75

Zaritsky, D., Kennicutt, Jr., R. C., \& Huchra, J. P. 1994, ApJ, 420, 87 
1 European Southern Observatory, 3107 Alonso de Córdova, Vitacura, Casilla 19001, Santiago 19, Chile

e-mail: t.kruehler@gmail.com

2 Dark Cosmology Centre, Niels Bohr Institute, University of Copenhagen, Juliane Maries Vej 30, 2100 København Ø, Denmark

3 Astronomical Institute Anton Pannekoek, University of Amsterdam, Science Park 904, 1098 XH Amsterdam, The Netherlands

${ }^{4}$ Centre for Astrophysics and Cosmology, Science Institute, University of Iceland, Dunhagi 5, 107 Reykjavik, Iceland

5 Department of Astronomy, California Institute of Technology, MC 249-17, 1200 East California Blvd, Pasadena, CA 91125, USA

6 INAF-IASF Bologna, Area della Ricerca CNR, via Gobetti 101, 40129 Bologna, Italy

7 Thüringer Landessternwarte Tautenburg, Sternwarte 5, 07778 Tautenburg, Germany

${ }^{8}$ Max-Planck-Institut für extraterrestrische Physik, Giessenbachstraße, 85748 Garching, Germany

9 Instituto de Astrofísica, Facultad de Física, Pontificia Universidad Católica de Chile, Vicuña Mackenna 4860, 7820436 Macul, Santiago, Chile

10 Millennium Institute of Astrophysics, Vicuña Mackenna 4860, 7820436 Macul, Santiago, Chile

11 Department of Physics and Astronomy, University of Leicester, University Road, Leicester, LE1 7RH, UK

12 Laboratoire Galaxies Étoiles Physique et Instrumentation, Observatoire de Paris, 5 place Jules Janssen, 92195 Meudon, France

13 INAF, Osservatorio Astronomico di Brera, via E. Bianchi 46, 23807 Merate (LC), Italy
14 American River College, Physics and Astronomy Dpt., 4700 College Oak Drive, Sacramento, CA 95841, USA

15 INAF, Osservatorio Astronomico di Roma, via Frascati 33, 00040 Monteporzio Catone, Italy

16 ASI-Science Data Centre, via Galileo Galilei, 00044 Frascati, Italy

17 Instituto de Astrofísica de Andalucía (IAA-CSIC), Glorieta de la Astronomía s/n, 18008 Granada, Spain

18 Institute of Experimental and Applied Physics, Czech Technical University in Prague, Horska 3a/22, 12800 Prague 2, Czech Republic

19 APC, Univ. Paris Diderot, CNRS/IN2P3, CEA/Irfu, Obs. de Paris, Sorbonne Paris Cité, 75205 Paris Cedex 13, France

${ }^{20}$ Faculty of Mathematics and Physics, University of Ljubljana, Jadranska ulica 19, 1000 Ljubljana, Slovenia

${ }^{21}$ Department of Physics, University of Warwick, Coventry CV4 7AL, UK

22 Department of Particle Physics \& Astrophysics, Weizmann Institute of Science, 76100 Rehovot, Israel

23 INAF, Osservatorio Astronomico di Trieste, via G.B. Tiepolo 11, 34143 Trieste, Italy

${ }^{24}$ European Southern Observatory, Karl-Schwarzschild-Strasse 2, 85748 Garching bei München, Germany

25 Scuola Normale Superiore, Piazza dei Cavalieri 7, 56126 Pisa, Italy

26 Unidad Asociada Grupo Ciencias Planetarias (UPV/EHU, IAACSIC), Departamento de Física Aplicada I, E.T.S. Ingeniería, Universidad del País Vasco (UPV/EHU), Alameda de Urquijo s/n, 48013 Bilbao, Spain

27 Ikerbasque, Basque Foundation for Science, Alameda de Urquijo 36-5, 48008 Bilbao, Spain

28 Physics Department, University of Calabria, via P. Bucci, 87036 Arcavacata di Rende, Italy 
Table 1. X-Shooter spectroscopic observations.

\begin{tabular}{|c|c|c|c|c|c|c|c|c|c|}
\hline \multirow[t]{2}{*}{ GRB host } & \multirow{2}{*}{$\begin{array}{c}\text { Gal. } E_{B-V} \\
\text { (mag) }\end{array}$} & \multicolumn{3}{|c|}{ Exposure time (s) } & \multicolumn{3}{|c|}{ Slit width } & \multirow[t]{2}{*}{ Obs. date } & \multirow[t]{2}{*}{ References } \\
\hline & & UVB & VIS & NIR & UVB & VIS & NIR & & \\
\hline GRB 050416A & 0.026 & $4 \times 900$ & $4 \times 900$ & $12 \times 300$ & $1{ }^{\prime \prime} 0$ & 0.9 & $0 ! 9$ & 2011-Jan.-19 & $(1),(2)$ \\
\hline \multirow[t]{2}{*}{ GRB $050525 \mathrm{~A}$} & 0.082 & $4 \times 1050$ & $4 \times 1050$ & $12 \times 350$ & 1.0 & 0.9 & $0.9 J H$ & 2012-Sep.-18 & (1) \\
\hline & & $4 \times 630$ & $4 \times 664$ & $4 \times 695$ & 1.0 & 0.9 & $0.9 \mathrm{JH}$ & 2012-Sep.-21 & \\
\hline GRB 050714B & 0.048 & $12 \times 940$ & $12 \times 900$ & $36 \times 325$ & $0 .{ }^{\prime} 8$ & 0.7 & 0.'6 & 2013-Mar.-19 & (1) \\
\hline GRB 050819 & 0.100 & $2 \times 900$ & $2 \times 900$ & $4 \times 450$ & 1.0 & 0.9 & 0.9 & 2010-Oct.-29 & $(1),(3)$ \\
\hline GRB 050824 & 0.030 & $4 \times 900$ & $4 \times 900$ & $8 \times 450$ & 1..0 & 0.9 & $0.9 \mathrm{JH}$ & 2011-Sep.-06 & (1) \\
\hline GRB 050915A & 0.022 & $6 \times 1200$ & $6 \times 1200$ & $12 \times 600$ & $1 . .0$ & 0.9 & $0.9 J H$ & 2011-Sep.-06 & $(1),(3),(4),(5)$ \\
\hline GRB 051001 & 0.013 & $2 \times 1800$ & $2 \times 1800$ & $4 \times 900$ & 1.0 & 0.9 & $0.9 \mathrm{JH}$ & 2010-Oct.-30 & (1), (3) \\
\hline GRB 051016B & 0.035 & $2 \times 600$ & $2 \times 600$ & $4 \times 300$ & 1.0 & 0.9 & 0.9 & 2009-Dec.-23 & (1) \\
\hline GRB 051022A & 0.052 & $2 \times 1200$ & $2 \times 1200$ & $4 \times 600$ & 1..6 & 1..5 & $0.9 \mathrm{JH}$ & 2012-Nov.-13 & (6) \\
\hline GRB 051117B & 0.048 & $1 \times 720$ & $1 \times 720$ & $2 \times 360$ & $1^{\prime \prime} 0$ & 0.9 & 0.9 & 2009-Dec.-23 & (1) \\
\hline GRB 060204B & 0.015 & $4 \times 900$ & $4 \times 900$ & $9 \times 300$ & $1 . .0$ & 0.9 & 0.9 & 2015-Mar.-05 & \\
\hline GRB 060306 & 0.030 & $4 \times 1800$ & $4 \times 1800$ & $12 \times 600$ & $1 . .3$ & 1 1.2 & 1..2 & 2011-Sep.-01 & $(1),(3),(5)$ \\
\hline GRB 060604 & 0.037 & $4 \times 1800$ & $4 \times 1800$ & $8 \times 900$ & 1..0 & 0.9 & 0.9 & 2010-Oct.-30 & $(1),(3)$ \\
\hline GRB 060707 & 0.019 & $4 \times 860$ & $4 \times 850$ & $12 \times 300$ & $1 . .3$ & 1.22 & $1^{\prime \prime} 2$ & 2009-Oct.-22 & $(1),(7)$ \\
\hline GRB 060719 & 0.007 & $4 \times 1500$ & $4 \times 1500$ & $12 \times 500$ & 1..0 & 0.9 & 0.9 & 2011-Oct.-20 & $(1),(3),(5)$ \\
\hline \multirow[t]{2}{*}{ GRB 060729} & 0.048 & $4 \times 810$ & $4 \times 844$ & $4 \times 875$ & 1..0 & 0.9 & $0.9 J H$ & 2012-Sep.-21 & (1) \\
\hline & & $4 \times 520$ & $4 \times 554$ & $4 \times 585$ & $1^{\prime \prime} 0$ & 0.9 & $0.9 \mathrm{JH}$ & 2012-Sep.-21 & \\
\hline GRB 060805A & 0.021 & $10 \times 940$ & $10 \times 900$ & $30 \times 320$ & $0 . ' 8$ & 0.7 & 0.6 & 2013-Mar.-19 & $(1),(3)$ \\
\hline GRB 060814 & 0.034 & $2 \times 1800$ & $2 \times 1800$ & $6 \times 600$ & 1.0 & 0.9 & 0.9 & 2010-Feb.-17 & $(1),(3),(5)$ \\
\hline GRB 060912A & 0.045 & $2 \times 900$ & $2 \times 934$ & $2 \times 965$ & 1.. 3 & 1..2 & 1."2 & 2012-Sep.-21 & (1) \\
\hline GRB 060923B & 0.129 & $2 \times 1800$ & $2 \times 1800$ & $6 \times 600$ & $1 . .0$ & 0.9 & $0.9 J H$ & 2013-Mar.-20 & (1) \\
\hline GRB 060926 & 0.137 & $4 \times 1800$ & $4 \times 1800$ & $12 \times 600$ & 1.0 & 0.9 & 0.9 & 2011-Apr.-24 & (18) \\
\hline GRB 061021 & 0.049 & $12 \times 940$ & $12 \times 900$ & $36 \times 320$ & 1..0 & 0.9 & $0.9 J H$ & 2013-Mar.-21 & (1) \\
\hline GRB 061110A & 0.076 & $4 \times 1800$ & $4 \times 1800$ & $12 \times 600$ & $1^{\prime \prime} 0$ & 0.9 & $0.9 \mathrm{JH}$ & 2011-Oct.-20 & (1) \\
\hline GRB 061202 & 0.131 & $4 \times 900$ & $4 \times 900$ & $9 \times 300$ & 1.0 & 0.9 & 0.9 & 2014-Nov.-29 & \\
\hline GRB 070103 & 0.058 & $2 \times 900$ & $2 \times 900$ & $4 \times 450$ & 1..0 & 0.9 & 0.9 & 2010-Oct.-29 & $(1),(3)$ \\
\hline GRB 070110 & 0.013 & $4 \times 1680$ & $4 \times 1680$ & $8 \times 840$ & $1 . .0$ & 0.9 & 0.9 & 2010-Oct.-29 & $(1),(7)$ \\
\hline GRB 070129 & 0.120 & $4 \times 900$ & $4 \times 900$ & $12 \times 300$ & 1.0 & 0.9 & 0.9 & 2009-Dec.-23 & (1) \\
\hline \multirow[t]{2}{*}{ GRB 070224} & 0.049 & $8 \times 940$ & $8 \times 900$ & $24 \times 300$ & 1.0 & 0.9 & 0.9 & 2013-Mar.-20 & (1), (3) \\
\hline & & $4 \times 900$ & $4 \times 920$ & $12 \times 320$ & 1..0 & 0.9 & 0.9 & 2013-Mar.-20 & \\
\hline GRB 070306 & 0.024 & $6 \times 900$ & $6 \times 900$ & $6 \times 900$ & 1.0 & 0.9 & 0.9 & 2010-Dec.-09 & $(1),(5),(8),(9)$ \\
\hline GRB 070318 & 0.015 & $4 \times 910$ & $4 \times 885$ & $12 \times 300$ & 1.0 & 0.9 & 0.9 & 2010-Oct.-06 & (1) \\
\hline \multirow[t]{2}{*}{ GRB 070328} & 0.031 & $7 \times 844$ & $7 \times 810$ & $8 \times 875$ & 1..0 & 0.9 & $0.9 J H$ & 2012-Sep.-21 & (1) \\
\hline & & $1 \times 589$ & $1 \times 585$ & & $1 . .0$ & 0.9 & $0.9 J H$ & 2012-Sep.-21 & \\
\hline GRB 070419B & 0.076 & $4 \times 1800$ & $4 \times 1800$ & $12 \times 600$ & $1^{\prime \prime} 0$ & 0.9 & 0.9 & 2011-Oct.-20 & (1) \\
\hline \multirow[t]{2}{*}{ GRB 070521} & 0.023 & $4 \times 900$ & $4 \times 900$ & $12 \times 300$ & 1.0 & 0.9 & 0.9 & 2013-Apr.-03 & $(5),(10)$ \\
\hline & & $4 \times 900$ & $4 \times 900$ & $12 \times 300$ & 1..0 & 0.9 & 0.9 & 2013-May-07 & \\
\hline GRB 070802 & 0.023 & $4 \times 1800$ & $4 \times 1800$ & $8 \times 900$ & 1.0 & 0.9 & 0.9 & 2010-Oct.-30 & $(1),(5),(9),(11)$ \\
\hline GRB 071021 & 0.057 & $4 \times 1800$ & $4 \times 1800$ & $12 \times 600$ & 1.0 & 0.9 & 0.9 & 2011-Sep.-06 & (3), (5) \\
\hline GRB 080207 & 0.020 & $4 \times 1800$ & $4 \times 1800$ & $12 \times 600$ & 1..0 & 0.9 & 0.9 & 2011-Apr.-24 & $(3),(5),(10)$ \\
\hline GRB 080413B & 0.032 & $3 \times 1800$ & $3 \times 1800$ & $6 \times 900$ & 1..0 & 0.9 & 0.9 & 2010-Aug.-15 & (23) \\
\hline \multirow[t]{2}{*}{ GRB 080602} & 0.025 & $4 \times 900$ & $4 \times 900$ & $12 \times 300$ & $1^{\prime \prime} 0$ & 0.9 & 0.9 & 2013-Aug.-18 & (10) \\
\hline & & $4 \times 900$ & $4 \times 900$ & $12 \times 300$ & 1.0 & 0.9 & 0.9 & 2013-Aug.-19 & \\
\hline GRB 080605 & 0.118 & $4 \times 885$ & $4 \times 910$ & $12 \times 300$ & 1.0 & 0.9 & 0.9 & 2011-Apr.-26 & $(9),(12)$ \\
\hline GRB 080804 & 0.014 & $4 \times 1350$ & $4 \times 1350$ & $12 \times 450$ & 1.0 & 0.9 & 0.9 & 2012-Apr.-19 & \\
\hline GRB 080805 & 0.037 & $4 \times 885$ & $4 \times 910$ & $12 \times 300$ & 1.0 & 0.9 & 0.9 & 2011-Мay-13 & (9) \\
\hline \multirow[t]{2}{*}{ GRB 081109} & 0.017 & $2 \times 1200$ & $2 \times 1200$ & $4 \times 600$ & 1.. 6 & 1.. 5 & $0.9 J H$ & 2012-Oct.-13 & $(5),(9)$ \\
\hline & & $2 \times 1200$ & $2 \times 1200$ & $4 \times 600$ & 1.' 6 & 1..5 & $0.9 J H$ & 2012-Nov.-14 & \\
\hline GRB 081210 & 0.066 & $4 \times 900$ & $4 \times 900$ & $12 \times 300$ & 1.0 & $1 . .0$ & $0 ! 9$ & 2014-Oct.-19 & \\
\hline GRB 081221 & 0.019 & $4 \times 1800$ & $4 \times 1800$ & $12 \times 600$ & $1 . .3$ & 1 1.2 & $1 " .2$ & 2011-Sep.-01 & $(5),(14)$ \\
\hline GRB 090113 & 0.072 & $2 \times 1800$ & $2 \times 1800$ & $4 \times 900$ & 1..0 & 0.9 & $0.9 J H$ & 2011-Sep.-06 & (3) \\
\hline \multirow[t]{2}{*}{ GRB 090201} & 0.059 & $2 \times 1200$ & $2 \times 1200$ & $8 \times 300$ & $1^{\prime \prime} 0$ & 0.9 & 0.9 & 2012-Dec.-25 & \\
\hline & & $2 \times 1200$ & $2 \times 1200$ & $8 \times 300$ & 1..0 & 0.9 & 0.9 & 2012-Dec.-31 & \\
\hline GRB 090323 & 0.021 & $3 \times 1560$ & $3 \times 1560$ & $9 \times 520$ & 1.0 & 0.9 & 0.9 & 2011-Mar.-11 & (15) \\
\hline GRB 090407 & 0.059 & $4 \times 1800$ & $4 \times 1800$ & $8 \times 900$ & $0 . ' 8$ & 0.7 & 0.6 & 2010-Oct.-30 & $(3),(5)$ \\
\hline GRB 090926B & 0.020 & $2 \times 1200$ & $2 \times 1200$ & $8 \times 300$ & 1.0 & 0.9 & $0.9 \mathrm{JH}$ & 2012-Nov.-14 & (9) \\
\hline & & $2 \times 1200$ & $2 \times 1200$ & $8 \times 300$ & 1..0 & 0.9 & $0.9 \mathrm{JH}$ & 2012-Dec.-17 & \\
\hline
\end{tabular}

References. (1) Hjorth et al. (2012); (2) Soderberg et al. (2007); (3) Krühler et al. (2012b); (4) Ovaldsen et al. (2007); (5) Perley et al. (2013b); (6) Levesque et al. (2010a); (7) Milvang-Jensen et al. (2012); (8) Jaunsen et al. (2008); (9) Krühler et al. (2011); (10) Rossi et al. (2012); (11) Elíasdóttir et al. (2009); (12) Filgas et al. (2011); (13) Krühler et al. (2012a); (14) Salvaterra et al. (2012); (15) McBreen et al. (2010); (16) Wiersema et al. (2012a); (17) Vergani et al. (2011); (18) Starling et al. (2011); (19) Levesque et al. (2011); (20) Niino et al. (2012); (21) de Ugarte Postigo et al. (2011); (22) Greiner et al. (2013); (23) Elliott et al. (2013); (24) Levan et al. (2014b); (25) Schulze et al. (2014); (26) de Ugarte Postigo et al. (2013); (27) Krühler et al. (2013); (28) Friis et al. (2015); (29) Xu et al. (2013); (30) Greiner et al. (2014); (31) Schady et al. (2015); (32) Fynbo et al. (2014). 
Table 1. continued.

\begin{tabular}{|c|c|c|c|c|c|c|c|c|c|}
\hline \multirow{2}{*}{ GRB host } & \multirow{2}{*}{$\begin{array}{l}\text { Gal. } E_{B-V} \\
\text { (mag) }\end{array}$} & \multicolumn{3}{|c|}{ Exposure time (s) } & \multicolumn{3}{|c|}{ Slit width } & \multirow[t]{2}{*}{ Obs. date } & \multirow[t]{2}{*}{ References } \\
\hline & & UVB & VIS & NIR & UVB & VIS & NIR & & \\
\hline GRB 091018 & 0.025 & $4 \times 600$ & $4 \times 600$ & $4 \times 600$ & $\begin{array}{l}1^{\prime \prime} 0 \\
\end{array}$ & 0.9 & $0 ! 9$ & 2009-Oct.-19 & (16) \\
\hline GRB 091127 & 0.033 & $4 \times 1500$ & $4 \times 1500$ & $8 \times 750$ & 1.”0 & 0.9 & 0.9 & 2009-Dec.-02 & (17) \\
\hline GRB 100316D & 0.101 & $4 \times 1200$ & $4 \times 1200$ & $12 \times 400$ & $0 . ' 8$ & 0.7 & 0.9 & 2011-Apr.-03 & (18), (19) \\
\hline GRB 100418A & 0.063 & $4 \times 1200$ & $4 \times 1200$ & $8 \times 600$ & 1.0 & 0.9 & 0.9 & 2010-Apr.-21 & (20), (21) \\
\hline GRB 100424A & 0.029 & $8 \times 600$ & $8 \times 600$ & $8 \times 600$ & $1 . \prime 0$ & 0.9 & 0.9 & 2013-Mar.-11 & \\
\hline GRB 100508A & 0.024 & $2 \times 1200$ & $2 \times 1200$ & $8 \times 300$ & 1.”0 & 0.9 & $0.9 \mathrm{JH}$ & 2012-Dec.-25 & \\
\hline \multirow[t]{2}{*}{ GRB 100606A } & 0.023 & $4 \times 900$ & $4 \times 900$ & $9 \times 300$ & 1.”0 & 0.9 & 0.9 & 2014-Nov.-18 & \\
\hline & & $4 \times 900$ & $4 \times 900$ & $9 \times 300$ & 1.0 & 0.9 & 0.9 & 2014-Nov.-28 & \\
\hline GRB 100615A & 0.039 & $4 \times 1200$ & $4 \times 1200$ & $16 \times 300$ & 1.0 & 0.9 & $0.9 J H$ & 2013-Mar.-05 & \\
\hline GRB 100621A & 0.027 & $2 \times 1200$ & $2 \times 1200$ & $8 \times 300$ & 1.”0 & 0.9 & $0.9 \mathrm{JH}$ & 2012-Oct.-16 & $(9),(22)$ \\
\hline GRB 100724A & 0.037 & $4 \times 1200$ & $4 \times 1200$ & $12 \times 400$ & $0 . ' 8$ & 0.7 & 0.9 & 2011-Apr.-03 & \\
\hline GRB 100728A & 0.151 & $2 \times 1200$ & $2 \times 1200$ & $8 \times 300$ & $1 . .0$ & 0.9 & 0.9 & 2012-Nov.-15 & \\
\hline GRB 100814A & 0.017 & $4 \times 1200$ & $4 \times 1200$ & $8 \times 600$ & 1."0 & 0.9 & 0.9 & 2010-Aug.-18 & \\
\hline GRB 100816A & 0.074 & $4 \times 1200$ & $4 \times 1200$ & $8 \times 600$ & 1.'0 & 0.9 & 0.9 & 2010-Aug.-17 & \\
\hline GRB $110808 \mathrm{~A}$ & 0.009 & $4 \times 600$ & $4 \times 600$ & $4 \times 600$ & 1."0 & 0.9 & 0.9 & 2011-Aug.-08 & \\
\hline \multirow[t]{3}{*}{ GRB 110818A } & 0.030 & $4 \times 600$ & $4 \times 600$ & $4 \times 600$ & 1."0 & 0.9 & 0.9 & 2013-Мay-08 & \\
\hline & & $4 \times 600$ & $4 \times 600$ & $4 \times 600$ & 1.”0 & 0.9 & 0.9 & 2013-Мау-10 & \\
\hline & & $8 \times 600$ & $8 \times 600$ & $8 \times 600$ & $1 . .0$ & 0.9 & 0.9 & 2013-Jul.-04 & \\
\hline \multirow{3}{*}{ GRB 110918A } & 0.017 & $2 \times 1200$ & $2 \times 1200$ & $4 \times 600$ & 1.'6 & 1..5 & $0.9 \mathrm{JH}$ & 2012-Dec.-17 & (23) \\
\hline & & $2 \times 1200$ & $2 \times 1200$ & $4 \times 600$ & 1." 6 & 1..5 & $0.9 \mathrm{JH}$ & 2013-Jan.-07 & \\
\hline & & $2 \times 1200$ & $2 \times 1200$ & $4 \times 600$ & 1.'6 & 1..5 & $0.9 \mathrm{JH}$ & 2013-Jan.-16 & \\
\hline GRB 111123A & 0.047 & $4 \times 600$ & $4 \times 600$ & $4 \times 600$ & 1."0 & 0.9 & 0.9 & 2013-Apr.-07 & \\
\hline GRB 111129A & 0.036 & $4 \times 1200$ & $4 \times 1200$ & $16 \times 300$ & 1."0 & 0.9 & 0.9 & 2011-Apr.-29 & \\
\hline GRB 111209A & 0.015 & $4 \times 2400$ & $4 \times 2400$ & $16 \times 600$ & $1 . \prime 0$ & 0.9 & 0.9 & 2011-Dec.-29 & (24) \\
\hline GRB 111211A & 0.041 & $4 \times 600$ & $4 \times 600$ & $4 \times 600$ & 1..0 & 0.9 & 0.9 & 2011-Dec.-13 & \\
\hline GRB 111228A & 0.028 & $4 \times 600$ & $4 \times 600$ & $4 \times 600$ & 1.”0 & 0.9 & 0.9 & 2011-Dec.-29 & \\
\hline GRB 120118B & 0.124 & $6 \times 600$ & $6 \times 600$ & $6 \times 600$ & 1.”0 & 1..0 & $0.9 \mathrm{JH}$ & 2013-Feb.-13 & \\
\hline GRB 120119A & 0.096 & $6 \times 600$ & $6 \times 600$ & $6 \times 600$ & 1.”0 & 1.0 & $0 . ' 6 J H$ & 2013-Feb.-26 & \\
\hline GRB 120211A & 0.026 & $4 \times 1200$ & $4 \times 1200$ & $16 \times 300$ & 1.”0 & 0.9 & 0.9 & 2013-Feb.-17 & \\
\hline GRB 120224A & 0.032 & $4 \times 600$ & $4 \times 600$ & $4 \times 600$ & 1..0 & 0.9 & 0.9 & 2012-Feb.-25 & \\
\hline GRB 120422A & 0.030 & $4 \times 1200$ & $4 \times 1200$ & $16 \times 300$ & 1.0 & 0.9 & 0.9 & 2012-Apr.-22 & (25) \\
\hline \multirow[t]{2}{*}{ GRB 120624B } & 0.048 & $1 \times 1200$ & $1 \times 1200$ & $4 \times 300$ & 1.'0 & 0.9 & 0.9 & 2012-Jul.-12 & (26) \\
\hline & & $3 \times 1200$ & $3 \times 1200$ & $12 \times 300$ & 1."0 & 0.9 & 0.9 & 2012-Jul.-13 & \\
\hline GRB 120714B & 0.008 & $4 \times 1200$ & $4 \times 1200$ & $16 \times 300$ & 1.”0 & 0.9 & 0.9 & 2012-Jul.-15 & \\
\hline GRB 120722A & 0.042 & $2 \times 2400$ & $2 \times 2400$ & $8 \times 600$ & 1."0 & 0.9 & 0.9 & 2012-Jul.-23 & \\
\hline GRB 120805A & 0.027 & $4 \times 900$ & $4 \times 900$ & $4 \times 900$ & $1 . \prime 0$ & 1.0 & $0.9 J H$ & 2012-Sep.-15 & \\
\hline \multirow{3}{*}{ GRB 120815A } & 0.099 & $4 \times 600$ & $4 \times 600$ & $4 \times 600$ & 1."0 & 0.9 & 0.9 & 2013-Apr.-14 & (27) \\
\hline & & $4 \times 600$ & $4 \times 600$ & $4 \times 600$ & 1.”0 & 0.9 & 0.9 & 2013-Apr.-16 & \\
\hline & & $4 \times 600$ & $4 \times 600$ & $4 \times 600$ & 1."0 & 0.9 & 0.9 & 2013-Apr.-21 & \\
\hline GRB 121024A & 0.088 & $4 \times 600$ & $4 \times 600$ & $4 \times 600$ & 1."0 & 0.9 & 0.9 & 2012-Oct.-24 & (28) \\
\hline GRB 121027A & 0.017 & $14 \times 600$ & $14 \times 600$ & $14 \times 600$ & 1..0 0 & 0.9 & 0.9 & 2012-Nov.-30 & (23) \\
\hline GRB 121201A & 0.008 & $4 \times 1200$ & $4 \times 1200$ & $16 \times 300$ & 1.0 & 0.9 & $0.9 \mathrm{JH}$ & 2012-Dec.-02 & \\
\hline GRB 121209A & 0.039 & $2 \times 600$ & $2 \times 600$ & $2 \times 600$ & $1 . .0$ & 0.9 & 0.9 & 2012-Nov.-13 & \\
\hline GRB 130131B & 0.029 & $8 \times 900$ & $8 \times 900$ & $24 \times 300$ & 1..0 & 0.9 & $0.9 \mathrm{JH}$ & 2013-Mar.-09 & \\
\hline GRB 130427A & 0.017 & $2 \times 600$ & $2 \times 600$ & $2 \times 600$ & 1..0 & 0.9 & $0.9 \mathrm{JH}$ & 2013-Apr.-28 & (29) \\
\hline GRB 130701A & 0.073 & $2 \times 600$ & $2 \times 600$ & $2 \times 600$ & 1.0 & 0.9 & $0.9 \mathrm{JH}$ & 2013-Aug.-01 & \\
\hline GRB 130925A & 0.018 & $4 \times 1470$ & $4 \times 1500$ & $20 \times 300$ & 1."0 & 0.9 & $0.9 \mathrm{JH}$ & 2013-Oct.-25 & $(30),(31)$ \\
\hline GRB 131103A & 0.009 & $4 \times 600$ & $4 \times 600$ & $4 \times 600$ & 1.”0 & 0.9 & $0.9 \mathrm{JH}$ & 2013-Nov.-05 & \\
\hline GRB 131105A & 0.030 & $8 \times 600$ & $8 \times 600$ & $8 \times 600$ & $1 . \prime 0$ & 0.9 & 0.9 & 2013-Nov.-05 & \\
\hline GRB 131231A & 0.022 & $4 \times 600$ & $4 \times 600$ & $4 \times 600$ & $1 . .0$ & 0.9 & $0.9 J H$ & 2014-Feb.-01 & \\
\hline GRB 140114A & 0.014 & $6 \times 900$ & $6 \times 900$ & $18 \times 300$ & 1..0 & 0.9 & $0.9 \mathrm{JH}$ & 2014-Mar.-28 & \\
\hline GRB 140213A & 0.131 & $2 \times 600$ & $2 \times 600$ & $2 \times 600$ & $1 . .0$ & 0.9 & $0.9 \mathrm{JH}$ & 2014-Feb.-14 & \\
\hline GRB 140301A & 0.026 & $12 \times 600$ & $12 \times 600$ & $12 \times 600$ & 1..0 & 0.9 & $0.9 \mathrm{JH}$ & 2014-Mar.-02 & \\
\hline GRB 140430A & 0.117 & $2 \times 600$ & $2 \times 600$ & $2 \times 600$ & $1 . .0$ & 0.9 & 0.9 & 2014-Apr.-30 & \\
\hline \multirow{2}{*}{ GRB 140506A } & 0.082 & $8 \times 600$ & $8 \times 600$ & $8 \times 600$ & $1^{\prime \prime} 0$ & 0.9 & 0.9 & 2014-Мay-07 & $(32)$ \\
\hline & & $8 \times 600$ & $8 \times 600$ & $8 \times 600$ & 1.0 & 0.9 & 0.9 & 2014-Jun.-08 & \\
\hline
\end{tabular}


T. Krühler et al.: VLT/X-Shooter spectroscopy of GRB hosts

Table 2. Fluxes of Balmer lines for GRB hosts.

\begin{tabular}{|c|c|c|c|c|c|}
\hline GRB host & Redshift & $\mathrm{H} \delta$ & $\mathrm{H} \gamma$ & $\mathrm{H} \beta$ & $\mathrm{H} \alpha$ \\
\hline GRB 050416A & 0.6542 & $0.3 \pm 0.3 \pm 0.1$ & $1.4 \pm 0.3 \pm 0.2$ & $2.9 \pm 0.4 \pm 0.3$ & $16.0 \pm 2.0 \pm 1.6$ \\
\hline GRB $050525 \mathrm{~A}$ & 0.6063 & $0.1 \pm 0.1 \pm 0.1$ & $0.1 \pm 0.1 \pm 0.1$ & $0.3 \pm 0.2 \pm 0.1$ & $0.7 \pm 0.2 \pm 0.3$ \\
\hline GRB 050714B & 2.4383 & $\ldots$ & $0.6 \pm 0.3 \pm 0.2$ & $0.5 \pm 0.2 \pm 0.2$ & $3.4 \pm 0.6 \pm 0.8$ \\
\hline GRB 050819A & 2.5042 & $\cdots$ & $0.6 \pm 0.3 \pm 0.1$ & $1.0 \pm 0.3 \pm 0.2$ & $\ldots$ \\
\hline GRB 050824 & 0.8277 & $0.7 \pm 0.1 \pm 0.1$ & $1.1 \pm 0.1 \pm 0.2$ & $2.6 \pm 0.4 \pm 0.5$ & $6.8 \pm 0.6 \pm 1.3$ \\
\hline GRB 050915A & 2.5275 & $\ldots$ & $0.5 \pm 0.2 \pm 0.1$ & $1.8 \pm 0.2 \pm 0.4$ & \\
\hline GRB 051001 & 2.4295 & $\ldots$ & $1.3 \pm 0.6 \pm 0.3$ & $1.9 \pm 0.3 \pm 0.4$ & $13 \pm 4 \pm 2$ \\
\hline GRB 051016B & 0.9358 & $2.4 \pm 0.3 \pm 0.2$ & $6.3 \pm 0.4 \pm 0.5$ & $10.9 \pm 1.2 \pm 1.1$ & $40 \pm 6 \pm 4$ \\
\hline GRB 051022A & 0.8061 & $2.8 \pm 0.5 \pm 0.3$ & $8.1 \pm 0.8 \pm 0.7$ & $22.1 \pm 1.3 \pm 1.7$ & $110 \pm 4 \pm 15$ \\
\hline GRB 051117B & 0.4805 & $-1.4 \pm 0.8 \pm 0.1$ & $1.1 \pm 1.0 \pm 0.1$ & $3.0 \pm 1.0 \pm 0.2$ & $21.1 \pm 4.1 \pm 1.3$ \\
\hline GRB 060204B & 2.3393 & $\ldots$ & $\ldots$ & $4.1 \pm 1.1 \pm 0.6$ & $16.9 \pm 1.4 \pm 2.0$ \\
\hline GRB 060306 & 1.5597 & $\cdots$ & $\cdots$ & $1.4 \pm 2.0 \pm 0.4$ & $8.9 \pm 3.4 \pm 1.4$ \\
\hline GRB 060604 & 2.1355 & $\cdots$ & $\cdots$ & $0.4 \pm 0.1 \pm 0.1$ & $1.8 \pm 0.3 \pm 0.5$ \\
\hline GRB 060707 & 3.4246 & $\cdots$ & $\cdots$ & $1.1 \pm 1.8 \pm 0.8$ & \\
\hline GRB 060719 & 1.5318 & $\cdots$ & $0.1 \pm 0.5 \pm 0.1$ & $0.8 \pm 0.5 \pm 0.2$ & $3.8 \pm 0.4 \pm 0.7$ \\
\hline GRB 060729 & 0.5429 & $\cdots$ & $0.0 \pm 0.3 \pm 0.1$ & $0.6 \pm 0.2 \pm 0.1$ & $3.7 \pm 1.7 \pm 0.5$ \\
\hline GRB $060805 \mathrm{~A}$ & 2.3633 & $\cdots$ & $0.5 \pm 0.4 \pm 0.2$ & $1.4 \pm 0.3 \pm 0.4$ & $3.7 \pm 0.5 \pm 0.9$ \\
\hline GRB 060814 & 1.9223 & $\cdots$ & $\ldots$ & $8.3 \pm 3.0 \pm 1.5$ & $28 \pm 4 \pm 4$ \\
\hline GRB 060912A & 0.9362 & $0.5 \pm 0.3 \pm 0.1$ & $2.5 \pm 0.3 \pm 0.2$ & $5.9 \pm 0.5 \pm 0.5$ & $15.8 \pm 3.8 \pm 1.6$ \\
\hline GRB 060923B & 1.5094 & $\ldots$ & $\ldots$ & $-1.6 \pm 0.6 \pm 0.1$ & $2.6 \pm 0.8 \pm 0.3$ \\
\hline GRB 060926 & 3.2090 & $0.1 \pm 0.1 \pm 0.1$ & $\cdots$ & $0.3 \pm 0.2 \pm 0.2$ & 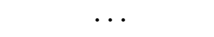 \\
\hline GRB 061021 & 0.3453 & $0.2 \pm 0.2 \pm 0.1$ & $0.2 \pm 0.2 \pm 0.1$ & $0.5 \pm 0.1 \pm 0.1$ & $1.9 \pm 0.1 \pm 0.2$ \\
\hline GRB 061110A & 0.7578 & $0.0 \pm 0.1 \pm 0.1$ & $\ldots$ & $0.3 \pm 0.1 \pm 0.1$ & 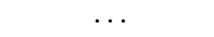 \\
\hline GRB 061202 & 2.2543 & $\ldots$ & $\cdots$ & $1.2 \pm 0.4 \pm 0.3$ & $6.1 \pm 0.8 \pm 1.3$ \\
\hline GRB 070103 & 2.6208 & $1.1 \pm 1.9 \pm 0.6$ & $3.9 \pm 1.6 \pm 1.2$ & $4.3 \pm 0.8 \pm 1.1$ & 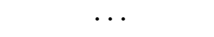 \\
\hline GRB 070110 & 2.3523 & $\ldots$ & $0.6 \pm 0.4 \pm 0.2$ & $\ldots$ & $3.5 \pm 0.6 \pm 0.8$ \\
\hline GRB 070129 & 2.3384 & $\cdots$ & $0.7 \pm 0.6 \pm 0.2$ & $1.5 \pm 0.9 \pm 0.4$ & $6.5 \pm 1.1 \pm 1.1$ \\
\hline GRB 070224 & 1.9922 & $\ldots$ & $0.1 \pm 0.1 \pm 0.1$ & $0.2 \pm 0.1 \pm 0.2$ & \\
\hline GRB 070306 & 1.4965 & $1.7 \pm 1.3 \pm 0.1$ & $7.7 \pm 3.6 \pm 0.8$ & $11.6 \pm 1.2 \pm 0.7$ & $53.5 \pm 1.4 \pm 3.7$ \\
\hline GRB 070318 & 0.8401 & $\ldots$ & $0.3 \pm 0.1 \pm 0.1$ & $1.2 \pm 0.2 \pm 0.2$ & $3.3 \pm 0.4 \pm 0.5$ \\
\hline GRB 070328 & 2.0627 & $0.3 \pm 0.2 \pm 0.1$ & $0.1 \pm 0.3 \pm 0.1$ & $1.1 \pm 0.2 \pm 0.2$ & \\
\hline GRB 070419B & 1.9586 & $\ldots$ & $\ldots$ & $0.9 \pm 0.3 \pm 0.3$ & $4.4 \pm 0.6 \pm 1.1$ \\
\hline GRB 070521 & 2.0865 & $\cdots$ & $\ldots$ & $-0.2 \pm 0.9 \pm 0.2$ & $10 \pm 5 \pm 3$ \\
\hline GRB 070802 & 2.4538 & $\cdots$ & $0.4 \pm 0.2 \pm 0.1$ & $1.4 \pm 0.2 \pm 0.3$ & $5.1 \pm 0.7 \pm 1.0$ \\
\hline GRB 071021 & 2.4515 & $\cdots$ & $0.7 \pm 0.5 \pm 0.2$ & $2.8 \pm 0.3 \pm 0.5$ & $8.9 \pm 1.8 \pm 1.8$ \\
\hline GRB 080207 & 2.0856 & $\cdots$ & $1.0 \pm 0.6 \pm 0.3$ & $1.3 \pm 0.5 \pm 0.3$ & $10.9 \pm 1.9 \pm 2.2$ \\
\hline GRB 080413B & 1.1012 & $\ldots$ & $0.2 \pm 0.1 \pm 0.1$ & $\ldots$ & $2.6 \pm 1.0 \pm 0.8$ \\
\hline GRB 080602 & 1.8204 & $1.6 \pm 0.9 \pm 0.3$ & $2.1 \pm 0.7 \pm 0.3$ & $\cdots$ & $30 \pm 8 \pm 4$ \\
\hline GRB 080605 & 1.6408 & $\ldots$ & $\ldots$ & $7.7 \pm 0.8 \pm 1.3$ & $29.1 \pm 1.4 \pm 4.3$ \\
\hline GRB 080804 & 2.2059 & $\cdots$ & $\cdots$ & $0.9 \pm 0.4 \pm 0.3$ & $3.7 \pm 0.8 \pm 1.2$ \\
\hline GRB 080805 & 1.5052 & $\cdots$ & $\cdots$ & $1.7 \pm 0.6 \pm 0.5$ & $11.0 \pm 1.6 \pm 2.7$ \\
\hline GRB 081109 & 0.9785 & . & $1.8 \pm 0.4 \pm 0.3$ & $5.5 \pm 0.5 \pm 0.8$ & $20.9 \pm 0.9 \pm 2.7$ \\
\hline GRB 081210 & 2.0631 & $0.3 \pm 0.3 \pm 0.1$ & $1.1 \pm 0.4 \pm 0.3$ & $2.1 \pm 0.3 \pm 0.5$ & $\ldots$ \\
\hline GRB 081221 & 2.2590 & $\ldots$ & $\ldots$ & $2.4 \pm 1.3 \pm 1.7$ & $9.5 \pm 1.9 \pm 5.3$ \\
\hline GRB 090113 & 1.7494 & $\cdots$ & $3.6 \pm 1.2 \pm 0.7$ & $3.3 \pm 0.9 \pm 0.6$ & $15 \pm 2 \pm 3$ \\
\hline GRB 090201 & 2.1000 & $\cdots$ & $3.8 \pm 1.8 \pm 1.1$ & $6.0 \pm 1.4 \pm 1.4$ & $23 \pm 2 \pm 4$ \\
\hline GRB 090323 & 3.5832 & $\cdots$ & $-0.3 \pm 0.4 \pm 0.1$ & $0.0 \pm 0.5 \pm 0.1$ & . \\
\hline GRB 090407 & 1.4478 & $-0.2 \pm 0.3 \pm 0.1$ & $-0.1 \pm 0.3 \pm 0.1$ & $0.7 \pm 0.2 \pm 0.1$ & $4.4 \pm 0.4 \pm 0.6$ \\
\hline GRB 090926B & 1.2427 & $0.5 \pm 0.2 \pm 0.1$ & $0.7 \pm 0.4 \pm 0.2$ & $2.7 \pm 0.9 \pm 0.5$ & $14.2 \pm 2.5 \pm 2.4$ \\
\hline GRB 091018 & 0.9710 & $\cdots$ & $0.0 \pm 0.2 \pm 0.1$ & $1.5 \pm 0.8 \pm 0.1$ & $4.2 \pm 0.6 \pm 0.3$ \\
\hline GRB 091127 & 0.4904 & $0.7 \pm 0.2 \pm 0.1$ & $0.7 \pm 0.2 \pm 0.1$ & $1.6 \pm 0.2 \pm 0.1$ & $5.5 \pm 0.3 \pm 0.5$ \\
\hline GRB $100316 D^{a}$ & 0.0592 & $14.0 \pm 0.7$ & $27.8 \pm 1.4$ & $62 \pm 3$ & $212 \pm 11$ \\
\hline
\end{tabular}

Notes. Measurements are in units of $10^{-17} \mathrm{erg} \mathrm{cm}^{-2} \mathrm{~s}^{-1}$, and are corrected for the Galactic foreground reddening. A correction for slit-loss based on broad-band photometry has been applied to the measurements as described in the text. These values thus represent host-integrated measurements except for GRB 100316D (see below). Line fluxes are not corrected for host-intrinsic extinction. The first error represents the statistical error due to photon statistics and line-flux measurement. The second error is the systematic error in the absolute flux calibration due to slit-loss and scaling to photometry. Redshifts are given in a heliocentric reference frame. No data means that either the wavelength range of the respective line is not covered, all data in that wavelength range have been excluded from the automated fitting procedure, or no meaningful constraints could be obtained for the given line. ${ }^{(a)}$ GRB $100316 \mathrm{D}$ has the lowest redshift in the sample $(z=0.0592)$. The fraction of the host that is covered by the slit is too small to derive representative host-integrated measurements. These values are thus as derived from the observed spectrum and not scaled by photometry. No systematic error on the line fluxes is given in this case. 
Table 2. continued.

\begin{tabular}{|c|c|c|c|c|c|}
\hline GRB host & Redshift & $\mathrm{H} \delta$ & $\mathrm{H} \gamma$ & $\mathrm{H} \beta$ & $\mathrm{H} \alpha$ \\
\hline GRB 100418A & 0.6235 & $1.6 \pm 1.3 \pm 0.3$ & $4.5 \pm 0.5 \pm 0.5$ & $10.0 \pm 0.7 \pm 1.0$ & $35 \pm 3 \pm 4$ \\
\hline GRB $100424 A$ & 2.4656 & $\ldots$ & $0.8 \pm 0.4 \pm 0.3$ & $2.0 \pm 0.2 \pm 0.5$ & $6.5 \pm 1.6 \pm 1.9$ \\
\hline GRB 100508A & 0.5201 & $\ldots$ & $1.0 \pm 0.9 \pm 0.1$ & $7.2 \pm 0.6 \pm 0.5$ & $24.9 \pm 1.8 \pm 1.6$ \\
\hline GRB 100606A & 1.5545 & $\ldots$ & $\ldots$ & $1.7 \pm 1.0 \pm 0.7$ & $4.7 \pm 0.5 \pm 1.4$ \\
\hline GRB 100615A & 1.3978 & $\cdots$ & $0.2 \pm 0.4 \pm 0.1$ & $1.0 \pm 0.4 \pm 0.2$ & $4.8 \pm 0.5 \pm 0.7$ \\
\hline GRB 100621A & 0.5426 & $\ldots$ & $18.8 \pm 0.8 \pm 1.1$ & $43.8 \pm 1.0 \pm 2.4$ & $128 \pm 5 \pm 7$ \\
\hline GRB 100724A & 1.2890 & $\ldots$ & $0.2 \pm 0.3 \pm 0.1$ & $1.1 \pm 0.7 \pm 0.5$ & $3.8 \pm 0.5 \pm 1.1$ \\
\hline GRB 100728A & 1.5670 & $\ldots$ & $1.2 \pm 2.5 \pm 1.4$ & $2.5 \pm 2.5 \pm 1.9$ & $10.6 \pm 1.7 \pm 4.7$ \\
\hline GRB 100814A & 1.4392 & $\cdots$ & $\ldots$ & $1.3 \pm 0.3 \pm 0.2$ & $4.0 \pm 0.3 \pm 0.4$ \\
\hline GRB 100816A & 0.8048 & $\ldots$ & $\ldots$ & $1.7 \pm 0.4 \pm 0.2$ & $19 \pm 2 \pm 3$ \\
\hline GRB 110808A & 1.3490 & $\cdots$ & $\cdots$ & $2.0 \pm 0.6 \pm 0.4$ & $7.6 \pm 0.5 \pm 1.1$ \\
\hline GRB 110818A & 3.3609 & $\cdots$ & $\cdots$ & $1.6 \pm 0.4 \pm 0.3$ & $\ldots$ \\
\hline GRB 110918A & 0.9843 & $\cdots$ & $-0.1 \pm 1.6 \pm 0.1$ & $10.3 \pm 2.4 \pm 0.6$ & $42 \pm 11 \pm 4$ \\
\hline GRB $111123 \mathrm{~A}$ & 3.1513 & $\ldots$ & $\ldots$ & $\ldots$ & $\ldots$ \\
\hline GRB 111129A & 1.0796 & $\ldots$ & $1.1 \pm 0.5 \pm 0.1$ & $3.3 \pm 1.9 \pm 0.4$ & $\cdots$ \\
\hline GRB 111209A & 0.6770 & $0.2 \pm 0.1 \pm 0.1$ & $0.3 \pm 0.1 \pm 0.1$ & $0.7 \pm 0.1 \pm 0.1$ & $2.4 \pm 0.6 \pm 0.5$ \\
\hline GRB 111211A & 0.4786 & $\ldots$ & $0.5 \pm 0.7 \pm 0.1$ & $-0.5 \pm 0.4 \pm 0.1$ & $2.3 \pm 0.4 \pm 0.2$ \\
\hline GRB 111228A & 0.7164 & $\ldots$ & $0.1 \pm 0.2 \pm 0.1$ & $0.5 \pm 0.3 \pm 0.1$ & $\ldots$ \\
\hline GRB 120118B & 2.9428 & $\ldots$ & $2.0 \pm 0.3 \pm 0.3$ & $2.4 \pm 0.8 \pm 0.3$ & $\ldots$ \\
\hline GRB 120119A & 1.7291 & $0.2 \pm 1.6 \pm 0.3$ & $1.5 \pm 0.7 \pm 0.4$ & $4.9 \pm 1.0 \pm 1.1$ & $19.2 \pm 1.1 \pm 3.7$ \\
\hline GRB 120422A & 0.2826 & $3.2 \pm 0.3 \pm 0.1$ & $6.4 \pm 0.5 \pm 0.3$ & $14.8 \pm 0.7 \pm 0.6$ & $57.5 \pm 1.1 \pm 2.1$ \\
\hline GRB 120624B & 2.1974 & $-0.2 \pm 0.6 \pm 0.1$ & $\ldots$ & $2.9 \pm 1.4 \pm 1.0$ & $10.3 \pm 0.7 \pm 3.1$ \\
\hline GRB 120714B & 0.3985 & $0.7 \pm 0.5 \pm 0.1$ & $0.5 \pm 0.3 \pm 0.1$ & $2.5 \pm 0.2 \pm 0.2$ & $7.5 \pm 0.3 \pm 0.5$ \\
\hline GRB 120722A & 0.9590 & $1.4 \pm 0.2 \pm 0.2$ & $2.6 \pm 0.2 \pm 0.3$ & $8.7 \pm 1.2 \pm 1.0$ & $32.0 \pm 1.5 \pm 3.4$ \\
\hline GRB $120815 A$ & 2.3587 & $\ldots$ & $\ldots$ & $0.3 \pm 0.1 \pm 0.1$ & $0.8 \pm 0.2 \pm 0.2$ \\
\hline GRB 121024A & 2.3012 & $\cdots$ & $\cdots$ & $7.3 \pm 0.6 \pm 0.6$ & $17.7 \pm 1.2 \pm 1.5$ \\
\hline GRB $121027 \mathrm{~A}$ & 1.7732 & $\ldots$ & $\cdots$ & $\ldots$ & $\ldots$ \\
\hline GRB $121201 \mathrm{~A}$ & 3.3830 & $0.6 \pm 0.4 \pm 0.1$ & $\cdots$ & $\ldots$ & $\ldots$ \\
\hline GRB 130131B & 2.5393 & $\ldots$ & $\ldots$ & $0.5 \pm 0.4 \pm 0.2$ & $\cdots$ \\
\hline GRB 130427A & 0.3401 & $-0.7 \pm 1.0 \pm 0.1$ & $0.3 \pm 1.1 \pm 0.1$ & $4.8 \pm 0.9 \pm 0.4$ & $14.6 \pm 1.3 \pm 1.1$ \\
\hline GRB $130701 \mathrm{~A}$ & 1.1548 & & & $1.3 \pm 2.4 \pm 0.2$ & $\ldots$ \\
\hline GRB $130925 \mathrm{~A}$ & 0.3483 & $1.2 \pm 1.0 \pm 0.1$ & $5.9 \pm 1.0 \pm 0.3$ & $11.7 \pm 0.7 \pm 0.6$ & $54 \pm 2 \pm 3$ \\
\hline GRB 131103A & 0.5960 & $3.1 \pm 1.1 \pm 0.6$ & $6.0 \pm 1.3 \pm 1.0$ & $20.2 \pm 1.1 \pm 2.8$ & $51 \pm 5 \pm 8$ \\
\hline GRB $131105 \mathrm{~A}$ & 1.6854 & $1.0 \pm 0.6 \pm 0.3$ & $0.7 \pm 0.6 \pm 0.3$ & $1.7 \pm 0.5 \pm 0.5$ & $10.1 \pm 0.7 \pm 2.3$ \\
\hline GRB 131231A & 0.6427 & $1.1 \pm 0.3 \pm 0.1$ & $2.4 \pm 0.3 \pm 0.2$ & $3.9 \pm 0.5 \pm 0.4$ & $14.1 \pm 1.1 \pm 1.4$ \\
\hline GRB 140213A & 1.2079 & $\ldots$ & $\ldots$ & $0.6 \pm 0.7 \pm 0.1$ & $1.2 \pm 0.5 \pm 0.1$ \\
\hline GRB $140301 \mathrm{~A}$ & 1.4155 & $2.1 \pm 0.6 \pm 0.2$ & $1.4 \pm 0.6 \pm 0.2$ & $5.0 \pm 0.6 \pm 0.5$ & $31.7 \pm 1.5 \pm 2.7$ \\
\hline GRB 140430A & 1.6019 & $\ldots$ & $\ldots$ & $\ldots$ & $5.8 \pm 0.8 \pm 0.6$ \\
\hline GRB 140506A & 0.8893 & $\ldots$ & $\cdots$ & $\ldots$ & $1.2 \pm 0.4 \pm 0.2$ \\
\hline
\end{tabular}


T. Krühler et al.: VLT/X-Shooter spectroscopy of GRB hosts

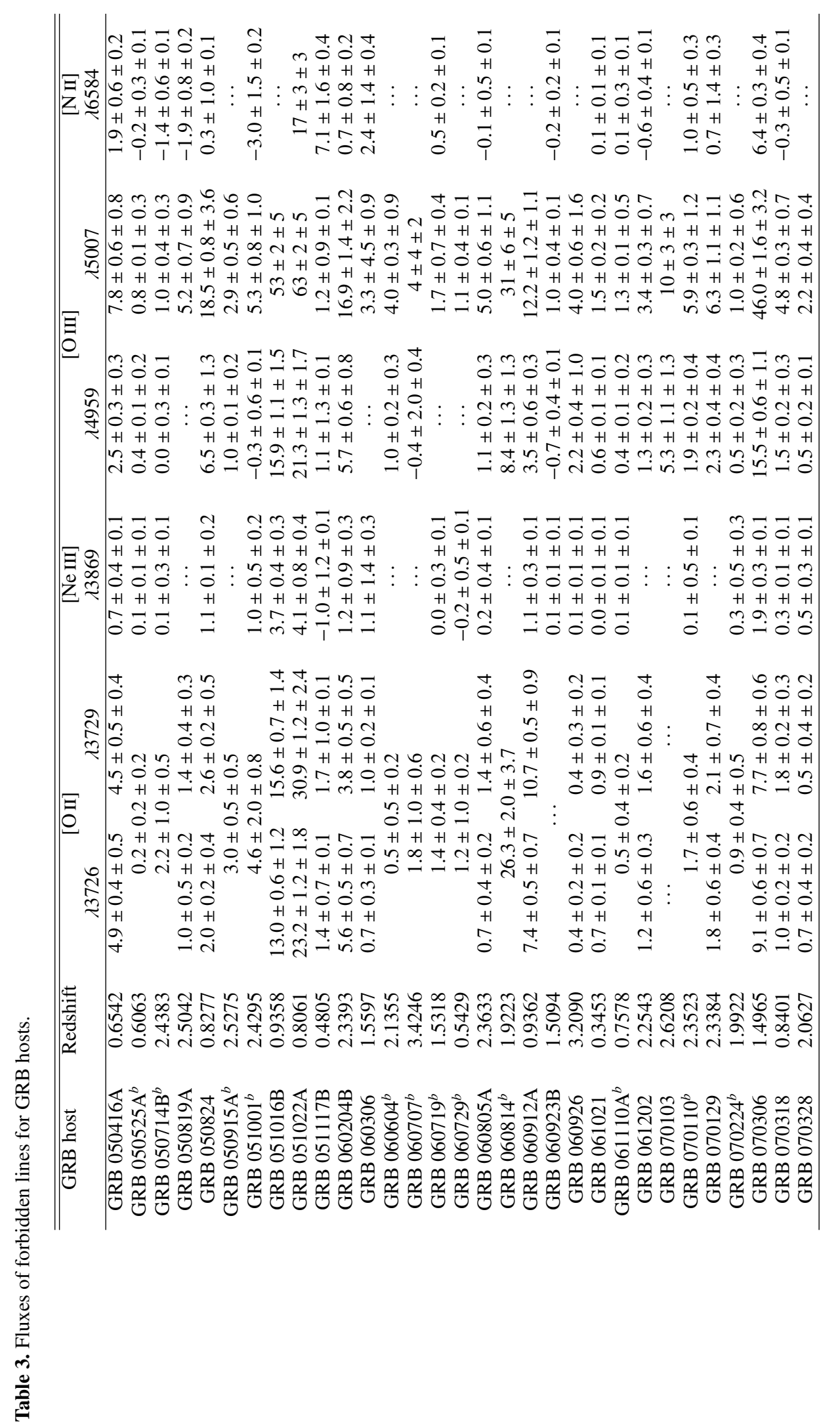

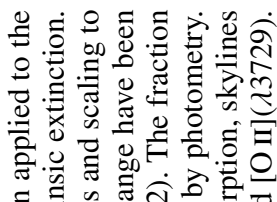

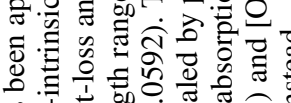

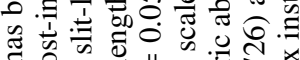

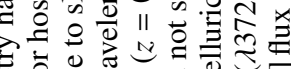

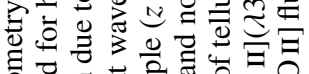

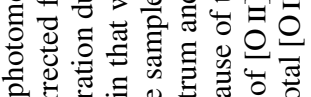

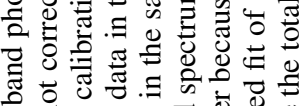

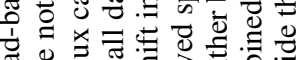

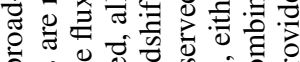

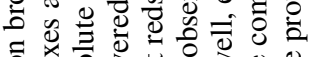
万人

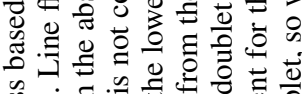

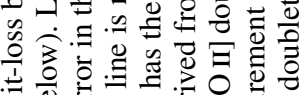

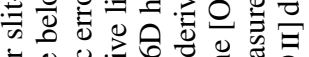

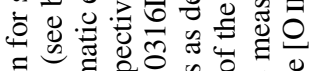
응 0 记

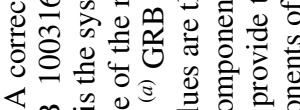
«i.

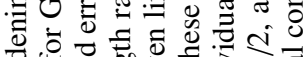

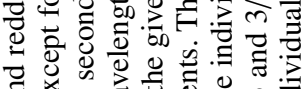
可 0 它记

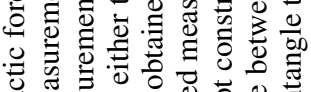

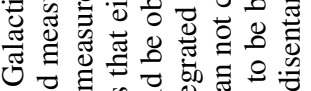
o

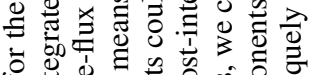

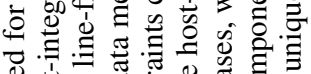

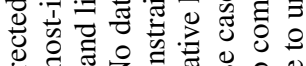

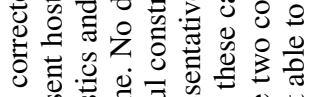

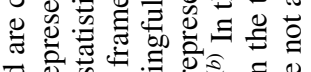

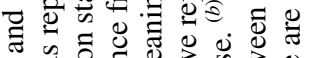

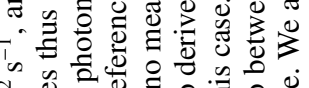
i 등

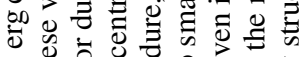
1)

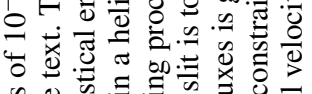

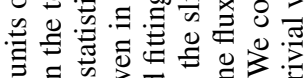

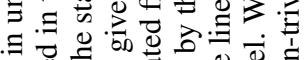

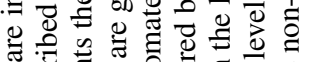

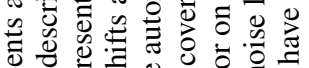

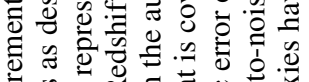

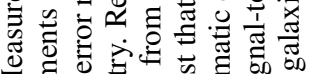
$\sum^{\circ} \Xi_{0} 0$

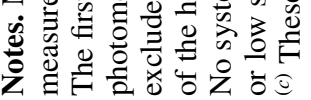




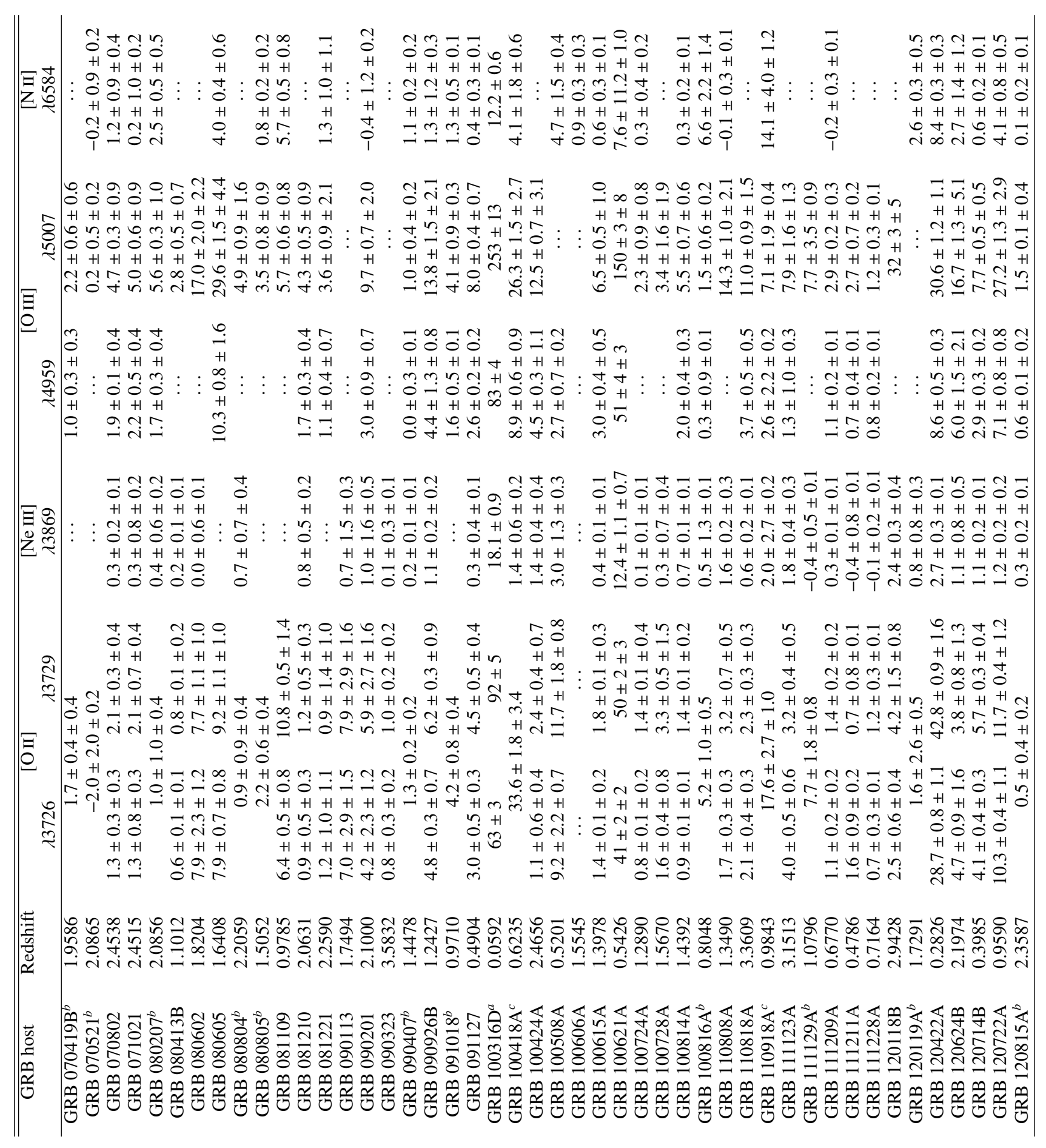




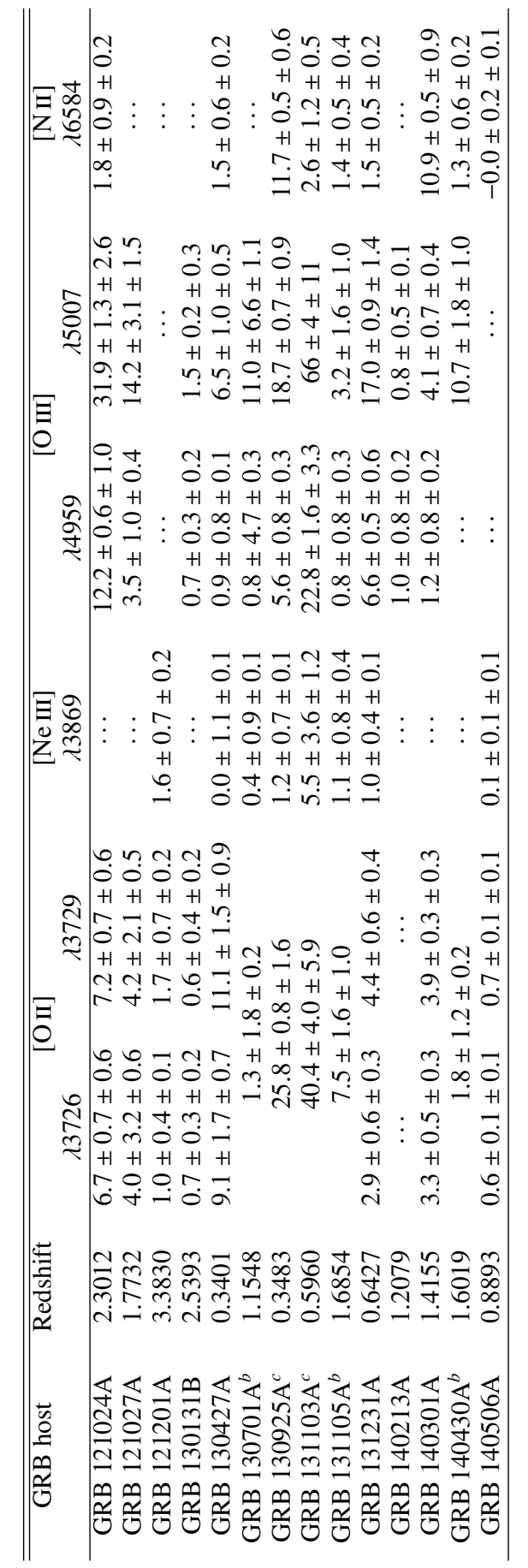


Table 4. Physical properties of GRB hosts.

\begin{tabular}{|c|c|c|c|c|c|c|}
\hline GRB host $^{a}$ & Redshift & $E_{B-V}$ & SF-tracer & $S F R^{b}$ & $\sigma$ & $Z$ \\
\hline & & (mag) & & $\left(M_{\odot} \mathrm{yr}^{-1}\right)$ & $\left(\mathrm{km} \mathrm{s}^{-1}\right)$ & $12+\log (\mathrm{O} / \mathrm{H})$ \\
\hline GRB 050416A & 0.6542 & $0.49_{-0.11}^{+0.11}$ & $\mathrm{H} \alpha$ & $4.5_{-1.2}^{+1.6}$ & $47 \pm 4$ & $8.46_{-0.11}^{+0.11}$ \\
\hline GRB 050525A & 0.6063 & $0.10_{-0.10}^{+0.11}$ & $\mathrm{H} \alpha$ & $0.07_{-0.05}^{+0.21}$ & $26 \pm 5$ & $\ldots$ \\
\hline GRB 050714B & 2.4383 & $0.21_{-0.21}^{+0.28}$ & $\mathrm{H} \alpha$ & $12.9_{-5.3}^{+14.0}$ & $35 \pm 16$ & $\ldots$ \\
\hline GRB 050819A & 2.5042 & $0.34_{-0.34}^{+0.84}$ & $\mathrm{H} \beta$ & $22_{-15}^{+426}$ & $50 \pm 6$ & $\ldots$ \\
\hline GRB 050824 & 0.8277 & $0.00_{-0.00}^{+0.07}$ & $\mathrm{H} \alpha$ & $1.20_{-0.26}^{+0.30}$ & $48 \pm 5$ & $8.11_{-0.20}^{+0.18}$ \\
\hline GRB 050915A & 2.5275 & $0.84_{-0.60}^{+0.61}$ & $\mathrm{H} \beta$ & $196_{-174}^{+1563}$ & $87 \pm 10$ & $\ldots$ \\
\hline GRB 051001 & 2.4295 & $0.58_{-0.28}^{+0.28}$ & $\mathrm{H} \alpha$ & $110_{-59}^{+124}$ & $67 \pm 8$ & $\ldots$ \\
\hline GRB 051016B & 0.9358 & $0.05_{-0.05}^{+0.07}$ & $\mathrm{H} \alpha$ & $10.2_{-2.0}^{+2.6}$ & $58 \pm 4$ & $8.27_{-0.20}^{+0.15}$ \\
\hline GRB 051022A & 0.8061 & $0.56_{-0.04}^{+0.05}$ & $\mathrm{H} \alpha$ & $60_{-10}^{+12.0}$ & $88 \pm 5$ & $8.49_{-0.09}^{+0.09}$ \\
\hline GRB 051117B & 0.4805 & $0.72_{-0.24}^{+0.27}$ & $\mathrm{H} \alpha$ & $4.7_{-2.2}^{+4.9}$ & $85 \pm 11$ & $9.00_{-0.16}^{+0.16}$ \\
\hline GRB 060204B & 2.3393 & $0.34_{-0.23}^{+0.29}$ & $\mathrm{H} \alpha$ & $78_{-34}^{+85}$ & $85 \pm 8$ & $\ldots$ \\
\hline GRB 060306 & 1.5597 & $0.44_{-0.40}^{+0.69}$ & $\mathrm{H} \alpha$ & $17.6_{-11.0}^{+83.6}$ & $60 \pm 14$ & $\ldots$ \\
\hline GRB 060604 & 2.1355 & $0.38_{-0.27}^{+0.32}$ & $\mathrm{H} \alpha$ & $7.2_{-3.6}^{+9.4}$ & $68 \pm 13$ & $8.10_{-0.35}^{+0.28}$ \\
\hline GRB 060707 & 3.4246 & $\ldots$ & [O II $]$ & $19.9_{-14.3}^{+48.0}$ & $\ldots$ & $\ldots$ \\
\hline GRB 060719 & 1.5318 & $0.40_{-0.36}^{+0.52}$ & $\mathrm{H} \alpha$ & $7.1_{-3.9}^{+18.9}$ & $42 \pm 5$ & $8.61_{-0.24}^{+0.20}$ \\
\hline GRB 060729 & 0.5429 & $0.71_{-0.47}^{+0.40}$ & $\mathrm{H} \alpha$ & $0.96_{-0.69}^{+2.21}$ & $66 \pm 16$ & $\ldots$ \\
\hline GRB 060805A & 2.3633 & $0.00_{-0.00}^{+0.16}$ & $\mathrm{H} \alpha$ & $9.0_{-2.5}^{+3.9}$ & $71 \pm 7$ & $\cdots$ \\
\hline GRB 060814 & 1.9223 & $0.17_{-0.17}^{+0.39}$ & $\mathrm{H} \alpha$ & $54_{-19}^{+89}$ & $132 \pm 11$ & $\ldots$ \\
\hline GRB 060912A & 0.9362 & $0.16_{-0.09}^{+0.10}$ & $\mathrm{H} \alpha$ & $5.1_{-1.6}^{+2.1}$ & $62 \pm 5$ & $8.61_{-0.12}^{+0.11}$ \\
\hline GRB 060923B & 1.5094 & $\ldots$ & $\mathrm{H} \alpha$ & $3.0_{-1.5}^{+2.9}$ & $46 \pm 15$ & $\ldots$ \\
\hline GRB 060926 & 3.2090 & $\ldots$ & [O III] & $26_{-17}^{+47}$ & $60 \pm 8$ & $\cdots$ \\
\hline GRB 061021 & 0.3453 & $0.11_{-0.11}^{+0.20}$ & $\mathrm{H} \alpha$ & $0.05_{-0.01}^{+0.03}$ & $16.1 \pm 4.8$ & $\cdots$ \\
\hline GRB 061110A & 0.7578 & $\ldots$ & $\mathrm{H} \beta$ & $0.23_{-0.15}^{+0.38}$ & $31 \pm 4$ & $\cdots$ \\
\hline GRB 061202 & 2.2543 & $0.58_{-0.27}^{+0.34}$ & $\mathrm{H} \alpha$ & $43_{-22}^{+60}$ & $64 \pm 7$ & $\cdots$ \\
\hline GRB 070103 & 2.6208 & $0.00_{-0.00}^{+0.50}$ & $\mathrm{H} \beta$ & $43_{-17}^{+162}$ & $124 \pm 30$ & $\cdots$ \\
\hline GRB 070110 & 2.3523 & $0.00_{-0.00}^{+0.38}$ & $\mathrm{H} \alpha$ & $8.9_{-2.8}^{+10.9}$ & $24 \pm 4$ & $\cdots$ \\
\hline GRB 070129 & 2.3384 & $0.17_{-0.17}^{+0.35}$ & $\mathrm{H} \alpha$ & $20_{-7}^{+28}$ & $76 \pm 11$ & $\cdots$ \\
\hline GRB 070224 & 1.9922 & $\ldots$ & [O III] & $3.2_{-2.3}^{+6.5}$ & $37 \pm 9$ & $\ldots$ \\
\hline GRB 070306 & 1.4965 & $0.43_{-0.07}^{+0.08}$ & $\mathrm{H} \alpha$ & $101_{-18}^{+24}$ & $121 \pm 55$ & $8.54_{-0.09}^{+0.09}$ \\
\hline GRB 070318 & 0.8401 & $0.15_{-0.14}^{+0.16}$ & $\mathrm{H} \alpha$ & $0.79_{-0.24}^{+0.44}$ & $53 \pm 5$ & $\ldots$ \\
\hline GRB 070328 & 2.0627 & $0.16_{-0.16}^{+0.79}$ & $\mathrm{H} \beta$ & $8.4_{-4.2}^{+130.7}$ & $93 \pm 14$ & $\cdots$ \\
\hline GRB 070419B & 1.9586 & $0.56_{-0.30}^{+0.39}$ & $\mathrm{H} \alpha$ & $21_{-11}^{+35}$ & $86 \pm 10$ & $\ldots$ \\
\hline GRB 070521 & 2.0865 & $\ldots$ & $\mathrm{H} \alpha$ & $26_{-17}^{+34}$ & $249 \pm 108$ & $\cdots$ \\
\hline GRB 070802 & 2.4538 & $0.31_{-0.12}^{+0.12}$ & $\mathrm{H} \alpha$ & $24_{-8}^{+11}$ & $57 \pm 5$ & $\cdots$ \\
\hline GRB 071021 & 2.4515 & $0.19_{-0.17}^{+0.16}$ & $\mathrm{H} \alpha$ & $32_{-12}^{+20}$ & $100 \pm 17$ & $\ldots$ \\
\hline GRB 080207 & 2.0856 & $0.66_{-0.25}^{+0.28}$ & $\mathrm{H} \alpha$ & $77_{-38}^{+86}$ & $136 \pm 18$ & $8.74_{-0.15}^{+0.15}$ \\
\hline GRB 080413B & 1.1012 & $0.43_{-0.32}^{+0.30}$ & $\mathrm{H} \alpha$ & $2.1_{-1.2}^{+3.1}$ & $39 \pm 5$ & $8.29_{-0.30}^{+0.32}$ \\
\hline GRB 080602 & 1.8204 & $0.58_{-0.26}^{+0.29}$ & $\mathrm{H} \alpha$ & $125_{-65}^{+145}$ & $91 \pm 13$ & $\ldots$ \\
\hline GRB 080605 & 1.6408 & $0.26_{-0.10}^{+0.11}$ & $\mathrm{H} \alpha$ & $47_{-12}^{+17}$ & $80 \pm 6$ & $8.54_{-0.09}^{+0.09}$ \\
\hline GRB 080804 & 2.2059 & $0.38_{-0.35}^{+0.51}$ & $\mathrm{H} \alpha$ & $15.2_{-8.7}^{+41.2}$ & $50 \pm 9$ & $\ldots$ \\
\hline GRB 080805 & 1.5052 & $0.78_{-0.31}^{+0.39}$ & $\mathrm{H} \alpha$ & $45_{-26}^{+79}$ & $54 \pm 12$ & $8.49_{-0.14}^{+0.13}$ \\
\hline GRB 081109 & 0.9785 & $0.36_{-0.10}^{+0.11}$ & $\mathrm{H} \alpha$ & $11.8_{-2.9}^{+4.1}$ & $108 \pm 6$ & $8.75_{-0.09}^{+0.09}$ \\
\hline
\end{tabular}

Notes. ${ }^{(a)}$ The physical parameters presented here are integrated and thus averaged over the entire galaxy. We do not perform a resolved analysis (neither spatially, nor in velocity space). In some cases (e.g., GRB 120422A, Schulze et al. 2014), a spatially resolved analysis leads to somewhat different results and interpretation of the galaxy properties. ${ }^{(b)}$ The quoted error on SFR is logarithmic because it contains the error in the dust correction. The derived SFR also has a lower limit because of the physical condition that $A_{V}>0$ mag. This lower limit is given by $S F R_{\min }=$ $4.8 \times \mathrm{F}_{\mathrm{H} \alpha, 42}$. 
T. Krühler et al.: VLT/X-Shooter spectroscopy of GRB hosts

Table 4. continued.

\begin{tabular}{|c|c|c|c|c|c|c|}
\hline GRB host ${ }^{a}$ & Redshift & $E_{B-V}$ & SF-tracer & $S F R^{b}$ & $\sigma$ & $Z$ \\
\hline & & (mag) & & $\left(M_{\odot} \mathrm{yr}^{-1}\right)$ & $\left(\mathrm{km} \mathrm{s}^{-1}\right)$ & $12+\log (\mathrm{O} / \mathrm{H})$ \\
\hline GRB 081210 & 2.0631 & $0.13_{-0.13}^{+0.61}$ & $\mathrm{H} \beta$ & $15.3_{-7.0}^{+111.7}$ & $118 \pm 12$ & $\ldots$ \\
\hline GRB 081221 & 2.2590 & $0.31_{-0.31}^{+0.55}$ & $\mathrm{H} \alpha$ & $35_{-22}^{+106}$ & $93 \pm 12$ & $\cdots$ \\
\hline GRB 090113 & 1.7494 & $0.01_{-0.01}^{+0.22}$ & $\mathrm{H} \alpha$ & $17.9_{-4.8}^{+10.1}$ & $70 \pm 9$ & $\cdots$ \\
\hline GRB 090201 & 2.1000 & $0.11_{-0.11}^{+0.19}$ & $\mathrm{H} \alpha$ & $\begin{array}{c}-4.0 \\
48_{-14}^{+30}\end{array}$ & $171 \pm 12$ & $\ldots$ \\
\hline GRB 090323 & 3.5832 & $\ldots$ & [O II $]$ & $24_{-17}^{+53}$ & $60 \pm 13$ & $\ldots$ \\
\hline GRB 090407 & 1.4478 & $0.69_{-0.26}^{+0.34}$ & $\mathrm{H} \alpha$ & $13.8_{-6.7}^{+18.8}$ & $109 \pm 8$ & $8.85_{-0.13}^{+0.13}$ \\
\hline GRB 090926B & 1.2427 & $0.63_{-0.18}^{+0.20}$ & $\mathrm{H} \alpha$ & $26_{-11}^{+19}$ & $65 \pm 4$ & $8.34_{-0.17}^{+0.15}$ \\
\hline GRB 091018 & 0.9710 & $0.06_{-0.06}^{+0.56}$ & $\mathrm{H} \alpha$ & $1.29_{-0.32}^{+3.46}$ & $57 \pm 10$ & $8.78_{-0.19}^{+0.18}$ \\
\hline GRB 091127 & 0.4904 & $0.16_{-0.08}^{+0.09}$ & $\mathrm{H} \alpha$ & $0.37_{-0.07}^{+0.10}$ & $30 \pm 5$ & $8.07_{-0.20}^{+0.18}$ \\
\hline GRB 100418A & 0.6235 & $0.17_{-0.07}^{+0.06}$ & $\mathrm{H} \alpha$ & $4.2_{-0.8}^{+1.0}$ & $56 \pm 4$ & $8.52_{-0.10}^{+0.20}$ \\
\hline GRB 100424A & 2.4656 & $0.13_{-0.13}^{+0.18}$ & $\mathrm{H} \beta$ & $21_{-8}^{+20}$ & $87 \pm 5$ & $7.93_{-0.18}^{+0.10}$ \\
\hline GRB 100508A & 0.5201 & $0.29_{-0.09}^{+0.09}$ & $\mathrm{H} \alpha$ & $2.6_{-0.5}^{+0.7}$ & $80 \pm 11$ & $8.68_{-0.10}^{-0.18}$ \\
\hline GRB 100606A & 1.5545 & $0.05_{-0.05}^{+0.60}$ & $\mathrm{H} \alpha$ & $4.9_{-1.8}^{+12.9}$ & $107 \pm 36$ & $8.71_{-0.21}^{+0.19}$ \\
\hline GRB 100615A & 1.3978 & $0.48_{-0.28}^{+0.38}$ & $\mathrm{H} \alpha$ & $8.6_{-4.4}^{+1.9}$ & $45 \pm 5$ & $8.40_{-0.13}^{+0.12}$ \\
\hline GRB 100621A & 0.5426 & $0.05_{-0.03}^{+0.03}$ & $\mathrm{H} \alpha$ & $8.7_{-0.8}^{-0.8}$ & $82 \pm 4$ & $8.52_{-0.10}^{+0.10}$ \\
\hline GRB 100724A & 1.2890 & $0.24_{-0.24}^{+0.37}$ & $\mathrm{H} \alpha$ & $3.2_{-1.4}^{+5.1}$ & $58 \pm 7$ & $\ldots$ \\
\hline GRB 100728A & 1.5670 & $0.23_{-0.23}^{+0.69}$ & $\mathrm{H} \alpha$ & $14.5_{-8.0}^{+60.6}$ & $57 \pm 8$ & $\cdots$ \\
\hline GRB 100814A & 1.4392 & $0.08_{-0.08}^{+0.26}$ & $\mathrm{H} \alpha$ & $3.2_{-0.7}^{+2.0}$ & $31 \pm 5$ & $\ldots$ \\
\hline GRB 100816A & 0.8048 & $1.32_{-0.22}^{+0.24}$ & $\mathrm{H} \alpha$ & $58_{-26}^{+51}$ & $111 \pm 30$ & $8.75_{-0.18}^{+0.16}$ \\
\hline GRB 110808A & 1.3490 & $0.30_{-0.25}^{+0.34}$ & $\mathrm{H} \alpha$ & $8.3_{-3.6}^{+11.3}$ & $40 \pm 4$ & $7.93_{-0.23}^{+0.31}$ \\
\hline GRB 110818A & 3.3609 & $\ldots$ & $\mathrm{H} \beta$ & $44_{-26}^{+62}$ & $89 \pm 8$ & $8.25_{-0.25}^{+0.17}$ \\
\hline GRB 110918A & 0.9843 & $0.35_{-0.31}^{+0.31}$ & $\mathrm{H} \alpha$ & $23_{-11}^{+28}$ & $126 \pm 18$ & $8.93_{-0.11}^{+0.11}$ \\
\hline GRB 111123A & 3.1513 & $\ldots$ & [O II $]$ & $77_{-52}^{+163}$ & $135 \pm 21$ & $8.01_{-0.28}^{+0.28}$ \\
\hline GRB 111129A & 1.0796 & $\ldots$ & [O II $]$ & $5.1_{-3.4}^{+10.8}$ & $117 \pm 35$ & $\ldots$ \\
\hline GRB 111209A & 0.6770 & $0.16_{-0.16}^{+0.20}$ & $\mathrm{H} \alpha$ & $0.35_{-0.13}^{+0.26}$ & $35 \pm 5$ & $7.95_{-0.17}^{+0.30}$ \\
\hline GRB 111211A & 0.4786 & $0.00_{-0.00}^{+0.10}$ & $\mathrm{H} \alpha$ & $0.12_{-0.03}^{+0.13}$ & $38 \pm 8$ & $\ldots$ \\
\hline GRB 111228A & 0.7164 & $\ldots$ & $\mathrm{H} \beta$ & $0.32_{-0.22}^{+0.56}$ & $19.7 \pm 5.5$ & $\ldots$ \\
\hline GRB 120118B & 2.9428 & $0.00_{-0.00}^{+0.16}$ & $\mathrm{H} \beta$ & $28_{-11}^{+21}$ & $193 \pm 8$ & $7.89_{-0.17}^{+0.23}$ \\
\hline GRB 120119A & 1.7291 & $0.35_{-0.14}^{+0.16}$ & $\mathrm{H} \alpha$ & $43_{-14}^{+24}$ & $104 \pm 17$ & $8.60_{-0.14}^{+0.14}$ \\
\hline GRB 120422A & 0.2826 & $0.27_{-0.03}^{+0.03}$ & $\mathrm{H} \alpha$ & $1.38_{-0.12}^{+0.13}$ & $25 \pm 4$ & $8.39_{-0.09}^{+0.09}$ \\
\hline GRB 120624B & 2.1974 & $0.21_{-0.21}^{+0.50}$ & $\mathrm{H} \alpha$ & $30_{-13}^{+73}$ & $77 \pm 6$ & $8.43_{-0.27}^{+0.20}$ \\
\hline GRB 120714B & 0.3985 & $0.10_{-0.08}^{+0.08}$ & $\mathrm{H} \alpha$ & $0.27_{-0.05}^{+0.07}$ & $34 \pm 4$ & $8.39_{-0.11}^{+0.11}$ \\
\hline GRB 120722A & 0.9590 & $0.46_{-0.05}^{+0.05}$ & $\mathrm{H} \alpha$ & $22_{-4}^{+4}$ & $56 \pm 4$ & $8.48_{-0.10}^{+0.10}$ \\
\hline GRB 120815A & 2.3587 & $0.06_{-0.06}^{+0.34}$ & $\mathrm{H} \alpha$ & $2.3_{-1.0}^{+2.7}$ & $28 \pm 5$ & $\ldots$ \\
\hline GRB 121024A & 2.3012 & $0.00_{-0.00}^{+0.12}$ & $\mathrm{H} \alpha$ & $37_{-4}^{+4}$ & $88 \pm 4$ & $8.41_{-0.12}^{+0.11}$ \\
\hline GRB 121027A & 1.7732 & $\ldots$ & [O III] & $24_{-15}^{+41}$ & $119 \pm 75$ & $\ldots$ \\
\hline GRB 121201A & 3.3830 & $\cdots$ & [O II] & $30_{-21}^{+68}$ & $86 \pm 17$ & $\cdots$ \\
\hline GRB 130131B & 2.5393 & $\ldots$ & [O III $]$ & $8.0_{-5.0}^{+13.4}$ & $73 \pm 29$ & $\ldots$ \\
\hline GRB 130427A & 0.3401 & $0.06_{-0.06}^{+0.19}$ & $\mathrm{H} \alpha$ & $0.34_{-0.06}^{+0.20}$ & $40 \pm 5$ & $8.57_{-0.13}^{+0.12}$ \\
\hline GRB $130701 \mathrm{~A}$ & 1.1548 & $\ldots$ & [O II $]$ & $0.78_{-0.60}^{+2.03}$ & $82 \pm 42$ & $\ldots$ \\
\hline GRB 130925A & 0.3483 & $0.41_{-0.06}^{+0.06}$ & $\mathrm{H} \alpha$ & $2.9_{-0.4}^{+0.5}$ & $49 \pm 5$ & $8.73_{-0.08}^{+0.08}$ \\
\hline GRB 131103A & 0.5960 & $0.06_{-0.06}^{+0.07}$ & $\mathrm{H} \alpha$ & $4.4_{-0.9}^{+1.4}$ & $87 \pm 7$ & $8.48_{-0.12}^{+0.10}$ \\
\hline GRB 131105A & 1.6854 & $0.53_{-0.18}^{+0.21}$ & $\mathrm{H} \alpha$ & $31_{-13}^{+25}$ & $52 \pm 11$ & $8.61_{-0.20}^{+0.17}$ \\
\hline GRB 131231A & 0.6427 & $0.02_{-0.02}^{+0.08}$ & $\mathrm{H} \alpha$ & $1.38_{-0.20}^{+0.28}$ & $33 \pm 4$ & $8.45_{-0.12}^{+0.11}$ \\
\hline GRB 140213A & 1.2079 & $0.06_{-0.06}^{+0.72}$ & $\mathrm{H} \alpha$ & $0.72_{-0.34}^{+2.65}$ & $34 \pm 14$ & $\ldots$ \\
\hline GRB 140301A & 1.4155 & $0.75_{-0.10}^{+0.11}$ & $\mathrm{H} \alpha$ & $106_{-25}^{+36}$ & $117 \pm 6$ & $8.89_{-0.09}^{+0.09}$ \\
\hline GRB 140430A & 1.6019 & $\ldots$ & $\mathrm{H} \alpha$ & $8.5_{-3.8}^{+7.1}$ & $40 \pm 7$ & $8.67_{-0.19}^{+0.18}$ \\
\hline GRB 140506A & 0.8893 & $\cdots$ & $\mathrm{H} \alpha$ & $0.35_{-0.19}^{+0.35}$ & $61 \pm 9$ & $\ldots$ \\
\hline
\end{tabular}




\section{Appendix A: Notes on individual targets}

\section{A.1. GRB 111129A}

The X-Shooter spectrum of the GRB 111129A afterglow (Racusin et al. 2011) was taken on 2011-Nov.-30, approximately $8.3 \mathrm{~h}$ after the initial Swift trigger. Above a red continuum (Rossi et al. 2011) we detect a single emission line (significance of $5.4 \sigma$ ) at a wavelength of $7756 \AA$. Interpreting this as [O II], the redshift of GRB 111129A is $z=1.0796$. If it were any of the other strong lines, we would expect to detect at least one other emission line in the spectrum, which we do not. At $z=1.0796$, however, there are indications for [O III] and $\mathrm{H} \beta$ at the $2 \sigma$ level in the NIR arm ( $\mathrm{H} \alpha$ is in the $J H$-bandgap and thus not seen).

\section{A.2. GRB $120211 A$}

In deep Keck imaging taken on 2013-Feb.-10, we detect an $I_{\mathrm{AB}}=25.0 \pm 0.2$ mag host galaxy candidate within the XRT error circle (Goad et al. 2012) of GRB 120211A (Sonbas et al. 2012). The spectral continuum of the putative host galaxy is detected also at high significance in the X-Shooter spectrum from 2013-Mar.-20 in a wavelength range between $9000 \AA$ and $4100 \AA$. Below $4100 \AA$ the flux sharply drops to zero. This apparent break is consistent with the onset of Ly $\alpha$ absorption at $z \sim 2.4$. Consistent with this redshift, there are minor indications at the $2 \sigma$-significance level for [O III] $] 55007$ emission at $z=2.346$ located in a region of high sky emission.

\section{A.3. GRB 120224A}

In the X-Shooter spectrum (Wiersema et al. 2012b) of an afterglow candidate of GRB 120224A (Saxton et al. 2012) taken on 2012-Feb.-25, we detect a red continuum in the VIS and NIR arms, without obvious emission lines. Later GROND imaging reveals this to be a very red $\left(r-K_{\mathrm{AB}}=3.7 \mathrm{mag}\right)$, and constant source, a plausible GRB host candidate. The spectral continuum drops below the noise floor blueward of $7000 \AA$. We rule out that the break is due to the Ly $\alpha$ at $z=4.8$ because the photometric colors, in particular the $g$-band detection $\left(g^{\prime}=25.2 \pm 0.2\right)$ disfavors such a high redshift. A photometric redshift analysis gives $90 \%$ confidence interval of $0.9<z_{\text {phot }}<1.3$. Indeed, there is a small redshift window at $z \sim 1.1$ in which all strong emission lines ([O II], [O III] and $\mathrm{H} \alpha$ ) would be hidden in telluric bands. This would provide an explanation for the non-detection of emission lines in our spectrum. The drop to zero flux in the spectrum would in this case be caused by intrinsic redness of the galaxy and the Balmer break. Significantly higher or lower redshifts are unlikely: The stellar mass of the galaxy derived from the photometry exceeds $10^{11} M_{\odot}$ already at $z>1.5$, making the nondetection of emission lines hard to explain. Similarly, at $z \lesssim 0.6$, we would have expected to detect at least $\mathrm{H} \alpha$ in the spectrum even for a dusty galaxy.

\section{A.4. GRB $120805 A$}

The galaxy coincident with the afterglow (Guidorzi \& Mundell 2012; Gorosabel et al. 2012) of GRB 120805A (Troja et al. 2012) is identified through NOT (Malesani et al. 2012) and late GROND and VLT/HAWK-I observations at a brightness of $r=24.1 \pm 0.1 \mathrm{mag}$. It was observed with X-Shooter spectroscopically on 2012-Aug.-15. The spectral continuum is detected at high significance in the UVB/VIS arm between $5000 \AA$ and $9500 \AA$. No signal is recorded blueward of $5000 \AA$ or in the NIR arms. The shape of the spectral continuum and the GROND photometry are consistent with a redshift of $z \sim 3.1$. The Ly $\alpha$ break explains the drop in flux at around $5000 \AA$, and the absence of strong emission lines in the spectrum is caused by telluric absorption bands. At this redshift, the host is luminous: $M_{1700 \AA} \sim-21.9 \mathrm{mag}$ corresponds to $\sim 2.5 M^{*}$ at $z \sim 3$ (e.g., Reddy \& Steidel 2009).

\section{A.5. GRB 121209A}

The $R_{\mathrm{AB}}=24.1 \pm 0.1 \mathrm{mag}$ host galaxy of GRB $121209 \mathrm{~A}$ (Maselli et al. 2012) was identified already very early (Krühler et al. 2012c; Perley et al. 2012), and observed with X-Shooter on 2012-Dec.-13. The galaxy-continuum is detected significantly with X-Shooter between $4900 \AA$ and $9500 \AA$ but not redward and with Keck LRIS between the atmospheric cutoff and $10300 \AA$ (Perley et al. 2012). Using all available late photometry from VLT/FORS2 and VLT/HAWK-I, the best-fit photometric redshift for this galaxy is $z_{\text {phot }}=2.1 \pm 0.3$, in agreement with earlier limits (Perley et al. 2012). Despite the bright continuum, no emission lines are seen. This is also consistent with $z_{\text {phot }} \sim 1.9$ because all strong emission lines ([O III], [O II], and $\mathrm{H} \alpha$ ) would be located in telluric absorption bands at this redshift.

\section{A.6. GRB $140114 A$}

At the position of the optical afterglow (Butler et al. 2014; Cano et al. 2014) of GRB 140114A (Troja et al. 2014), we detect an $R_{\mathrm{AB}}=24.4 \pm 0.2 \mathrm{mag}$ galaxy with NOT/ALFOSC that we interpret as the GRB's host galaxy. Our X-Shooter spectroscopy from 2014-Mar.-28 reveals no emission lines, but the galaxy continuum between $4850 \AA$ and $9800 \AA$. A sharp drop in flux blueward of this wavelength range is best described by the Ly $\alpha$ break at $z=3.0$. The relatively high redshift is also consistent with the absence of emission lines because the strong forbidden lines of [O II] and [O III] are in telluric absorption bands and $\mathrm{H} \alpha$ outside of the wavelength response of X-Shooter.

\section{Appendix B: Details on metallicity measurements and notable individual events}

To derive oxygen abundances via the strong-line diagnostic ratios from Nagao et al. (2006) and Maiolino et al. (2008), we take into account the dust attenuation, its uncertainty ([N II] versus $\mathrm{H} \alpha$ or [Ne III] versus [O II] have a negligible dependence on $A_{V}$ ) and the systematic scatter in the respective relation. We use $[\mathrm{N} \mathrm{II}] / \mathrm{H} \alpha$ if available to discriminate between the upper and lower $R_{23}$ branch. Table 4 provides the calculated metallicities and errors (as the range between $16 \%$ and $84 \%$ of cumulative probability distribution). These oxygen abundances need to be taken with a grain of salt, in particular at higher redshifts, as indicated in the main text.

In some cases, in particular at the highest redshifts $(z>2.5)$, dust corrections to the line fluxes are not available because of lacking Balmer lines. In those cases, however, metallicities are derived using line ratios that are only marginally sensitive to dust corrections such as [Ne III] over [O II]. The missing constraints on $E_{B-V}$ are thus not a strong concern for the metallicity measurement. The systematic and statistical error of the [Ne III] over [O II] ratio is large, and it does not provide strong additional constraints once [N II] -based line diagnostics are available. It is, however, the only accessible method at $z>3$ so we use it with 
the caveat that it is the least tested and used strong-line diagnostic ratio. It did provide a consistent metallicity in those cases where we could test it with other methods.

Figure B.1 shows several representative examples of metallicity determinations for six galaxies at different redshifts. We specifically pick GRB hosts or afterglows that were extensively discussed in the recent literature: GRB 070306 (Jaunsen et al. 2008; Krühler et al. 2011), GRB 080207 (Hunt et al. 2011; Svensson et al. 2012), GRB 091127 (Vergani et al. 2011), GRB 100621A (Greiner et al. 2013), GRB 111209A (Gendre et al. 2013; Levan et al. 2014b; Greiner et al. 2015b; Kann et al. 2015) and GRB 130427A (e.g., Xu et al. 2013; Perley et al. 2014). We will discuss them briefly in the following section as they are illustrative for the metallicity measurement procedure and interesting individually.

\section{B.1. GRB 070306}

GRB $070306(z=1.497)$ is a well-studied dusty GRB (Jaunsen et al. 2008), with a host galaxy that is detected throughout the electromagnetic spectrum (Hunt et al. 2014; Schady et al. 2014; Perley et al. 2015b). The X-Shooter spectrum shows numerous emission lines, which allow the Balmer decrement to be reasonably well measured $\left(E_{B-V}=0.43_{-0.07}^{+0.08} \mathrm{mag}\right)$. The $[\mathrm{N} \mathrm{II}] / \mathrm{H} \alpha$ lineflux ratio uniquely puts the $R_{23}$ solution onto the upper branch. The individual emission line diagnostics have overlapping probability distributions, and a simultaneous fit to all five line ratios results in a metallicity of $12+\log (\mathrm{O} / \mathrm{H})=8.54 \pm 0.09$, which is $0.8 \pm 0.2$ times the solar value (Fig. B.1).

\section{B.2. GRB 080207}

GRB $080207(z=2.086)$ is also a well-discussed dark GRB (Hunt et al. 2011; Svensson et al. 2012). Its host is detected by various facilities from the optical to radio (Rossi et al. 2012; Perley et al. 2013b), and has a very high stellar mass $\left(M_{\star} \sim\right.$ $10^{11} M_{\odot}$ ), extremely red optical/NIR colors and is vigorously forming stars $\left(S F R_{\mathrm{H} \alpha} \sim 90 M_{\odot} \mathrm{yr}^{-1}\right)$. Because of the large $A_{V} \sim 2$, [O II] is not detected and the applied strong-line diagnostics are thus based exclusively on [N II]. We measure an oxygen abundance around solar, $12+\log (\mathrm{O} / \mathrm{H})=8.74 \pm 0.15$. This is the GRB host with the highest metallicity at $z>2$ in the sample. Its metallicity, however, is averaged over the multiple knots that are detected at the spatial resolution of HST (Svensson et al. 2012), which in theory could host a range of metallicities.

\section{B.3. GRB 091127}

GRB $091127(z=0.490)$ is a typical low-redshift GRB with a bright afterglow and faint host (Vergani et al. 2011; Filgas et al. 2011). It is an example of a galaxy having $R_{23}$ in the overlap region between the two metallicity solutions. Even though the forbidden oxygen and Balmer lines are detected at good $\mathrm{S} / \mathrm{N}$, the lack of detection of either [Ne III] or [N II] limits the number of useful line-diagnostics to $R_{23}$. Given that the line ratios put the host of GRB 091127 at around the turn-over point of the two branches, the probability distribution is not double valued, but rather broad because $12+\log (\mathrm{O} / \mathrm{H})$ is not very sensitive to variations in $R_{23}$ in this region. We derive $12+$ $\log (\mathrm{O} / \mathrm{H})=8.07_{-0.20}^{+0.18}$ for the host of GRB 091127 .

\section{B.4. GRB $100621 A$}

GRB $100621 \mathrm{~A}(z=0.543)$ is a low- $z$ GRB with a faint and dust-reddened afterglow (Greiner et al. 2013). The host spectrum has, in addition to well-detected [O II], [O III], $\mathrm{H} \alpha$, and $\mathrm{H} \beta$ emission lines, a significant detection of [Ne III] (but not [N II]). The degeneracy of the double-peaked $R_{23}$ probability distribution in metallicity is clearly evident in Fig. B.1. It is, however, broken through [Ne III]. Even though the individual [Ne III]-based metallicity is not well constrained, in combination with $R_{23}$ we can derive $12+\log (\mathrm{O} / \mathrm{H})$ accurately to $12+\log (\mathrm{O} / \mathrm{H})=$ $8.52 \pm 0.10$ with only a very small remaining probability for the lower branch solution of the $R_{23}$ diagnostic.

\section{B.5. GRB 111209A}

GRB 111209A $(z=0.677)$ is one of the longest GRBs ever detected. The extreme temporal properties were used to postulate a new class of GRBs (Gendre et al. 2013; Levan et al. 2014b), a result questioned by Virgili et al. (2013). The ultralong duration is possibly indicative of a different progenitor channel (Nakauchi et al. 2013), and the associated luminous supernova is well-explained in a magnetar scenario (Kann et al. 2015; Greiner et al. 2015b). Based on the detection of [O II], [O III] and [Ne III], in addition to the Balmer lines, we measure $12+\log (\mathrm{O} / \mathrm{H})=7.95_{-0.17}^{+0.30}$ or between $10 \%$ and $40 \%$ of the solar value in the adopted metallicity scale. This puts the galaxy at the low end of metallicities compared to many GRBs of more normal duration at a similar redshift (Fig. 17). Together with the low reddening, narrow line width, compact morphology and low luminosity (Levan et al. 2014b) it shares many similarities with Blue Compact Dwarfs (e.g., Gil de Paz et al. 2003, and references therein) or hosts of super-luminous supernovae (e.g., Leloudas et al. 2015). The galaxy hosting GRB 111209A is, however, not extremely metal-poor, and its spectroscopic properties do not provide a stark contrast to long GRBs with a more common duration (see, e.g., GRB 091127 above), a conclusion similar to the one of Levan et al. (2014b).

\section{B.6. GRB $130427 A$}

GRB 130427A $(z=0.340)$ is one of the brightest GRBs of all time due to its low redshift and is extremely well studied from the radio to $\mathrm{GeV}$ energies (e.g., Perley et al. 2014; Ackermann et al. 2014; van der Horst et al. 2014). It is a very rare example of a low-redshift GRB with an energy budget comparable to common high-redshift GRBs and therefore elucidates cosmological GRBs and their connection to SNe through a local analogue (Xu et al. 2013; Levan et al. 2014a; Melandri et al. 2014). The host characteristics are relatively typical for the given redshift: With a $S F R_{\mathrm{H} \alpha}=0.3_{-0.1}^{+0.2} M_{\odot} \mathrm{yr}^{-1}$ and metallicity of $12+\log (\mathrm{O} / \mathrm{H})=8.57_{-0.13}^{+0.12}$ it is well within the distribution of host properties for low-redshift GRBs. 
A\&A 581, A125 (2015)
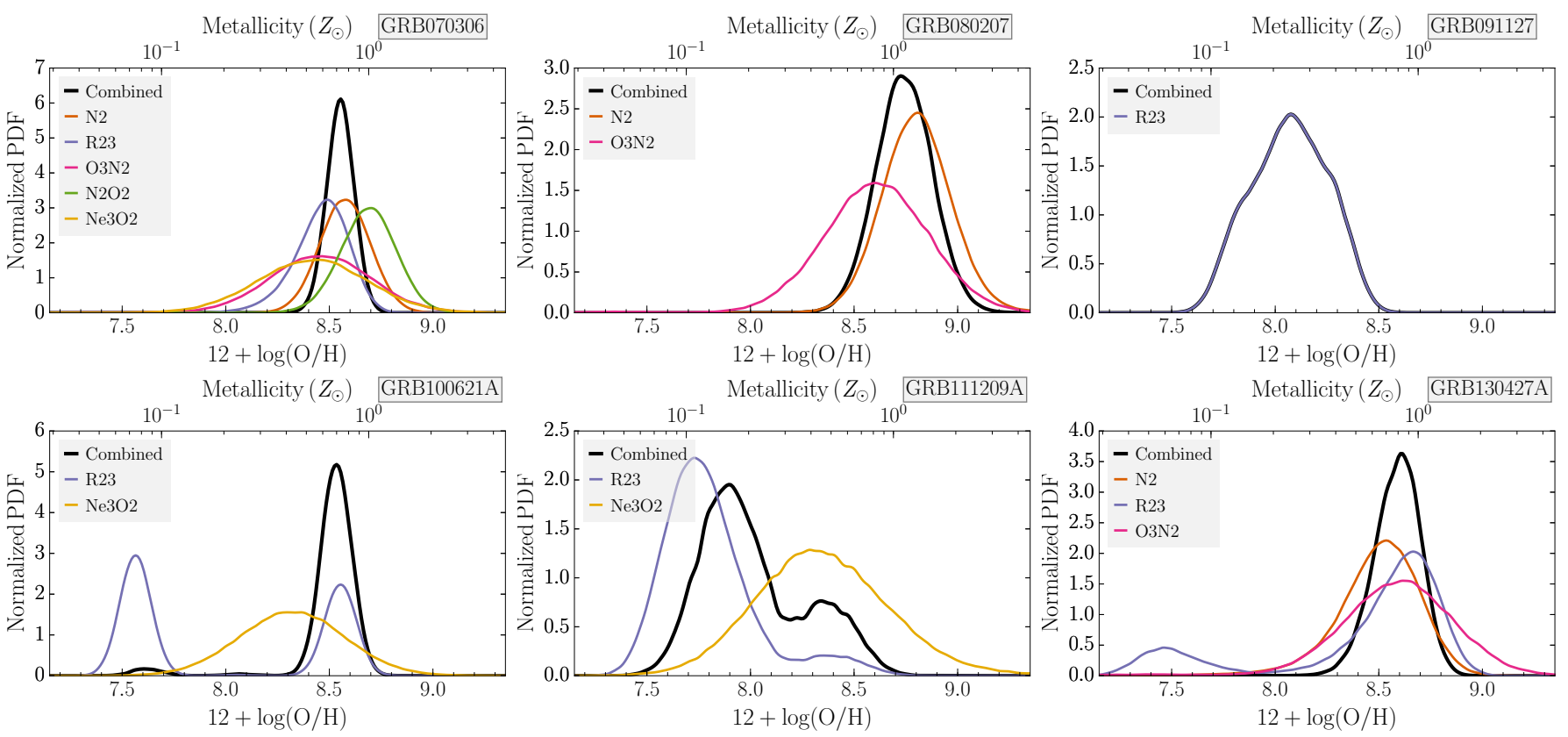

Fig. B.1. Example of metallicity measurements using different strong line diagnostics (Nagao et al. 2006; Maiolino et al. 2008). Every colored line represents the normalized probability distribution of one strong-line diagnostic. The black line is the probability distribution of $12+\log (\mathrm{O} / \mathrm{H})$ simultaneously minimized against all available measured line ratios. 